\title{
General sessions (1A-01 - 3E-12)
}

WS10 FUJITA, Miku ${ }^{1}$ ( ${ }^{1}$ The Third Dept. Agr., Univ. of $-7 \quad$ Hokkaido)

Report of the Activity of iGEM HokkaidoU

Circularization of a protein is one of the ways to enhance its stability against various temperatures and $\mathrm{pH}$ levels. We challenged ourselves to circularize proteins using self-assembling peptides and linkers. Self-assembling peptide (SAP) is an amphiphilic peptide which self-assembles under physicochemical conditions. We made SAPs fused to link ends of the protein. We also inserted cysteine residues in the linker which is essential for the circularization so that they form a disulfide bond. This linker is expected to be applicable to most proteins because it automatically adjusts its length. These two elements - the self-assembling peptide and the linker - enable the protein to be circularized.
$1 \mathrm{~A}$

$-01$

Canceled

1 A TANAKA, Seiji ${ }^{1,2}$, MIYAZAWA-ONAMI, Mayumi ${ }^{1}$, -02 HOSOE, Akari ${ }^{1}$, ARAKI, Hiroyuki ${ }^{1,2}$ ( ${ }^{1}$ Div. Microbial Genet., Natl. Inst. Genet., ${ }^{2}$ SOKENDAI)

Relationship between the initiation reaction of DNA replication and chromatin regulator in eukaryotes

DNA, the carrier of genetic information in organisms, is stored as chromosomes, which is consists from many chromatins, in nuclei in eukaryotes. It is widely known that there are regulatory mechanisms that cooperate chromatin regulating factors and DNA-mediated reactions, such as transcription and DNA repair. This can let us expect that the existence of cooperative mechanism(s) between chromatin regulators and DNA replication, which is a essential process for cell proliferation. However, this is largely unknown, although some study have shown that the contribution of chromatin regulators to replication licensing and elongation process of DNA replication. Moreover, the contribution of chromatin regulators onto the initiation reaction of DNA replication is totally unknown. Here we would like to show some results indicating a chromatin regulator has a role in the initiation of DNA replication. Our results reveal novel level of regulation of DNA replication. 
1 A SASAKI, Mariko ${ }^{1}$, KOBAYASHI, Takehiko ${ }^{1}\left({ }^{1}\right.$ Lab. -03 Genome Regen., Inst. Molec. Cell. BioSci., Univ. Tokyo)

Understanding the mechanisms that repair DNA doublestrand breaks arising from replication fork arrest

Arrested replication forks can lead to DNA double-strand breaks (DSBs). Replication-associated DSBs are a major source of genome rearrangements, which are a hallmark of cancer cells, cause human genomic disorders and influence genome diversity. Yet, it remains to be understood how replication-associated DSBs are repaired. In this study, we used the budding yeast ribosomal RNA gene locus to demonstrate that DSBs generated upon replication fork arrest are normally repaired by the pathways independent of homologous recombination (HR). In marked contrast, cells lacking a component of replisome $\mathrm{Ctf} 4$ require DSB end resection and subsequent recombination for DSB repair. These observations reveal that repair of DSBs arising from replication fork arrest is regulated differently from replicationindependent DSBs. Our findings illuminate a critical role of Ctf4 in protecting replication-associated DSBs from rearrangementprone HR pathways.

1 A MATSUZAKI, Kenichiro ${ }^{1}$, BOULTON, Simon ${ }^{2}\left({ }^{1}\right.$ Inst. -04 Prot. Res., Osaka Univ., ${ }^{2}$ The Francis Crick Inst.)

The Role of Fancj in the maintenance of microsatellites and lymphomagenesis

Maintenance of genome integrity during DNA replication is of vital importance to ensure that daughter cells inherit an intact copy of the genetic code. Repetitive DNA sequences are a particular challenge to genome stability due to their propensity to form secondary structures that hinder replisome progression and promote template slippage. Microsatellites are repetitive sequences of $1-10$ base pairs of DNA and highly prone to expansion/contraction due to their propensity to form non-B-form DNA structures. Although mismatch repair plays a key role in preventing microsatellite instability (MSI), activities must also exist that unwind secondary structures to facilitate replication fidelity.??In this study, we found that Fancj-deficient mice phenocopy other mouse models of Fanconi anemia (FA). Unexpectedly, Fancj-deficient mice also present with enhanced predisposition to lymphoma, and cells derived from these mice are hypersensitive to replication inhibitors and show high level of MSI. These data implicate Fancj as a key factor required to counteract MSI.?? We propose that FANCJ counteracts the formation of secondary structures that arise during replication of microsatellite.
1A HASEGAWA, Yuki ${ }^{1}$, KEYAMURA, Kenji ${ }^{1}$, HISHIDA, -05 Takashi ${ }^{1}\left({ }^{1}\right.$ Dept. Life Sci., Grad. Sch. Sci., Gakushuin Univ.)

The role of Mgs1 in DNA replication stress response in Saccharomyces cerevisiae

Saccharomyces cerevisiae Mgs1 protein possesses DNA binding and DNA-dependent ATPase activities, which plays a role in maintaining genome stability during DNA replication. The MGS1 orthologues are highly conserved in prokaryotes and eukaryotes. The yeast Mgs1 protein consists of a eukaryote-specific Nterminal domain containing a ubiquitin binding zinc finger motif, a central core $\mathrm{ATP}^{+} \mathrm{ATPase}$ domain, and a large C-terminal domain. In this study, we studied the role of C-terminal domain of Mgs1 by examining the effects of its C-terminal deletions on Mgs1 protein functions in vivo. We show that the expression of $\mathrm{C}$ terminal deletions results in higher sensitivity to methylmethane sulfonate compared with wild type Mgs1. This and other data suggest that the C-terminal deletions of Mgs1 have significant and complex effects on the activity of the protein in vivo.

1 A YOSHIYAMA, Kaoru Okamoto $^{1}$, KAMINOYAMA -06 Kaori $^{1}$, SAKAMOTO, Tomoaki ${ }^{1}$, KIMURA, Seisuke ( ${ }^{1}$ Life Sci.s, Kyoto Sangyo Univ.)

Mechanism of pathway selection in DNA damage responses in plants

Being sessile, plants are constantly exposed to various environmental stresses, such as ultraviolet from sun and reactive oxygen species produced by strong light, high temperature and drying stresses and so on. It is, thus, imperative that an efficient and specific DNA damage responses (DDRs) be in place in plants to cope with DNA damage. To understand plant DDRs, we have been focusing on Arabidopsis SOG1, a transcription factor, regulates more than 100 genes involved in the DDRs. SOG1 works as a master regulator of DDRs as it is required for various responses, including; the induction of cell cycle arrest, induction of DNA repair genes, induction of the endoreduplicative cell cycle and the induction of programmed cell death. However, it is unknown how these responses are regulated by this single master regulator. We previously showed that SQ motifs of SOG1 are phosphorylated in response to DNA damage and how the phosphorylation is essential for the activation of DDRs. We would like to report the relationship between the phosphorylation of SOG1 and the activation of various responses in this meeting. 
1 A HIRAI, Hirohisa ${ }^{1}$, HIRAI, Yuriko ${ }^{1}$, KOGA, Akihiko ${ }^{2}$ -07 ( ${ }^{1}$ Mol. Biol. Section, Primate Res. Inst., Kyoto Univ., ${ }^{2}$ Cell. Biol. Section, Primate Res. Inst., Kyoto Univ.)

De novo chromosomal alterations in interspecies hybrids of night monkeys

During chromosome analysis of 16 night (or owl) monkeys in Primate Res. Inst., Kyoto Univ., we encountered four individuals with unimaginable chromosome changes that are unexpected from a single species. Eventually, they were supposed to be chromosome modification induced by interspecies hybridization between Aotus azarae $(2 \mathrm{n}=49,50)$ and A. grisemembra $(2 n=53)$. They showed diploid chromosome numbers of 51, 52, 52, and 53, respectively. The former two showed chromosome organization predictable from their parents, but the latter two contained novel complicated chromosome rearrangements, showing trisomies of $\mathrm{X}$ - and 22-chromosomes and a translocation. To figure out the puzzle, we tried molecular cytogenetic analyses with chromosome paint and alpha satellite DNA probes.
1 MURATA, Minoru ${ }^{1}$, KANATANI, Asaka ${ }^{1}$, -08 KASHIHARA, Kazunari ${ }^{1}$, NAGAKI, Kiyotaka ${ }^{1}$ $\left({ }^{1}\right.$ Group of Nuclear Genomics, Inst. Plant Sci. and Resources, Okayama Univ.)

Transmission control of plant artificial ring chromosome in Arabidopsis thaliana

In the model plant, Arabidopsis thaliana, we have generated artificial ring chromosomes using the Cre/LoxP and Ac/Ds system. Despite the ring structure, they are stable during mitotic divisions and are transmissible to the next generation through meiosis. One of the artificial ring chromosomes, AtARC1, originated from a centromeric edge of the long arm of chromosome 2 , is approximately $2.85 \mathrm{Mb}$ in size. Although the centromeric 180 -bp repeat array of ARC1 is much shorter than that of the original chromosome $2(0.25 \mathrm{Mb}$ vs. $3.0 \mathrm{Mb})$, the transmission rates through the female and male sides were $11 \%$ and $28 \%$, respectively, when it was added to wild-type plants (Murata et al. 2103). In this study, ARC1 was transferred to a TDNA knockout line for Mcm9 (Minichromosome maintenance 9) gene that is expressed preferentially in pollens. As a result, the transmission of ARC1 increased to $100 \%$, because the $\mathrm{Mcm} 9$ gene on ARC1 complemented the recessive allele ( $\mathrm{mcm} 9$ ) on chromosome 2 and restored pollen viability. This suggests that the dose of $M c m 9$ gene in pollens affects the male-side transmission of ARC1.
1A KAWANO, Shinji ${ }^{1}$, FUJIMOTO, Kunpei ${ }^{1}$, YASUDA -09 Kazushi ${ }^{1}$, HAMADA, Ami ${ }^{1}$, TSUTSUI, Ken ${ }^{2}$, TSUTSUI, Kimiko M. ${ }^{2}$, IKEDA, Shogo ${ }^{1}$ ( ${ }^{1}$ Dept. Biochem., Faclt. Sci., Okayama Univ. of Sci., ${ }^{2}$ Dept. Neurogenomics, Grad. Sch. Med., Dent. and Pharm. Sci., Okayama Univ.)

Functional analysis of the C-terminal domain of DNA topoisomerase II

DNA topoisomerase II (topo II) is an essential enzyme for resolution of topological problems arising in DNA metabolic processes such as transcription and replication. We examined how the CTD of topo II is associated with DNA catenation activity by comparing WT rat topo II with its truncated mutant. The results indicate that DNA binding activity in the CTD of topo II concentrates the enzyme in the vicinity of condensed DNA and allows topo II to efficiently form a DNA catenane. $\begin{array}{ll}1 \mathrm{~A} & \text { OHTA, Shinya } \\ -10 & \left({ }^{1} \mathrm{Med} \text {. School, Kochi Univ. }\right)\end{array}$

Quantitative proteomics using chicken DT40 cells revealed novel mitotic chromosome scaffold component

Chromosome condensation is essential for proper segregation of the genome during mitosis, and gives chromosomes their characteristic shape. A classical model proposes that non-histone proteins act as a structural framework during the formation of mitotic chromosomes. This framework was suggested to correspond to an insoluble biochemical fraction, the chromosome scaffold, which can be derived from chromosomes after solubilization of the bulk of the DNA and proteins. Current works have shown that the components of the chromosomal scaffold fraction have an essential role in the formation of structurally stable mitotic chromosomes. However we do not yet have clear information about the existence of the chromosome scaffold. Here, we determined the protein composition of the chromosome scaffold using mass spectrometry and identified a novel chromosome scaffold component. This GFP-fusion protein localized to the mitotic chromosome axis. Moreover knocking out caused a mitotic progression error and slightly changed a mitotic chromosome shape. These results suggested that our novel protein might play a role in the chromosome scaffold for the determination of the mitotic chromosome shape. 
1B SHIMADA, Makoto K. ${ }^{1}\left({ }^{1}\right.$ Div. Gene Expr. Mech., Inst. -01 Compr. Med. Sci., Fujita Health Univ.)

Learning from the past: comparison among the known locus showing complex human history of admixture

Genomes of modern human populations include genomic fragment introgressed from archaic humans. Details of the introgression are still unknown.

To show the impact to genetic diversity of modern human (i.e., Homo sapiens) with examples, I selected 8 loci those had been discussed genomic introgression from non-modern humans before the publication of the Neanderthal genome, and evaluated them by gene genealogy and the statics for introgression ( $\mathrm{S}^{*}$ analysis) using sequences of Neanderthal, Denisova, and thousands of individuals of modern humans. These analyses show that at least three patterns in gene-genealogical topology of introgressed haplotypes suggested by $\mathrm{S}^{*}$ analysis. This may suggest several events of introgression from archaic humans before the Out-ofAfrica, which resulted in vaster diversity within modern human than expected from the introgression from the existing archaic human sequences.
1B FUJITO, Naoko ${ }^{1}$, SATTA, Yoko ${ }^{1}$, HANE, Masaya ${ }^{2}$, -03 MATSUI, Atsushi ${ }^{3}$, YASHIMA, Kenta ${ }^{1}$, KITAJIMA, Ken $^{2}$, SATO, Chihiro ${ }^{2}$, TAKAHATA, Naoyuki ${ }^{1}$, HAYAKAWA, Toshiyuki ${ }^{4}\left({ }^{1} \mathrm{Sch}\right.$. Adv. Sci., SOKENDAI (Grad. Univ. Adv. Stud.), ${ }^{2}$ Biosci. and BioTech. Center, Nagoya Univ., ${ }^{3}$ Primate Res. Inst., Kyoto Univ., ${ }^{4}$ Grad. Sch. Systems Life Sci.s, Kyushu Univ.)

The Out of Africa and mental disorders: Adaptation with reduction in STX promoter activity

STX is an enzyme responsible for transfer of polysialic acids to neural cell adhesion molecules and plays an important role in higher brain function of humans. Curiously, variants of the STX gene are often associated with various mental disorders. Here we focus on four SNP combinations (promoter types) composing of three SNPs (core SNPs) in the promoter region and show that one ("CGC") type with high frequency (35\%) in the Asian population has significantly lower promoter activity than the others. The phylogenetic study, based on haplotype sequences of 63 samples from various ethnic groups, reveals that the ancestral "CGC" emerged between 0.45 0.1 MYA and expanded around 0.1 MYA, which is coincident with the African exodus of modern humans. Further analysis using phased sequences from the 1000 genome project data (2504 individuals) reveals that "CGC" has a relatively long homozygosity tract with the average being $\sim 32$ $\mathrm{kb}$ from the core SNPs. This suggests action of positive selection and is consistent with results of REHH. It is argued that positive selection has played significant roles in shaping "CGC" in the Asian population throughout the Great Journey of modern humans.

1B SATO, Daiki ${ }^{1}$, MAKINO, Takashi ${ }^{1}$, KAWATA, -04 Masakado ${ }^{1}\left({ }^{1}\right.$ Dept. Ecol. Evolutionary Biol., Grad. Sch. of Life Sci., Tohoku Univ.)

Evolutionary genetic basis underlying social and cognitive abilities in human

Considering the fact that some psychiatric disorders have serious effects on patients' social or cognitive abilities, the genes underlying them should be key elements of the unique function in human brain. Here we focused on psychiatric disorders related genes and investigated the historical changes of the genes possibly contributing to human specificity by means of evolutionary bioinformatic approach. We found two human specific amino acid substitutions (Glu130Gly and Asp136Thr) in solute career family 18 member A1 (SLC18A1), which codes vesicular transporter of monoamine such as serotonin or dopamine, and these substitutions were predicted to have significant impact on protein function by both previous descriptions and our estimation. Moreover, there was a polymorphism on the 136th site (Thr136Ile), and we detected balancing selection acting on the site in non-African population. This suggests that the 136th site were likely to play important roles at the moment of the out of Africa. Our study proposes a new candidate gene putatively having impacts on the evolution of human brain through modulation of neurotransmission pathway. 
1B WADA, Yoshiko ${ }^{1,2}$, WADA, Kennosuke ${ }^{1}$, IWASAKI, -05 Yuki $^{1}$, KANAYA, Shigehiko ${ }^{2}$, IKEMURA, Toshimichi ${ }^{1}$ $\left({ }^{1}\right.$ Dept. BioSci, Nagahama Inst. Bio-Sci. Tech., ${ }^{2}$ Nara Inst. Sci. Tech.)

Big data analysis on rapidly evolving zoonotic RNA viruses and its use for establishment of the prediction verification cycle

Viruses have always posed a significant threat to public health, as exemplified by ebolavirus threat highlighted by recent outbreaks in West Africa. To face world-wide threats by infectious viruses (e.g. Ebola, influenza and MERS viruses), we must innovate various technologies including big data analysis. One important characteristics of these viruses is to have RNA genomes and to have very high evolutionary rates, showing importance of time-series analysis for developing defense strategies against these viruses. The present time-series analysis of oligonucleotide (e.g. 2 6-mers) composition by word count revealed directional changes in genome sequences after invasion from nonhuman animals, which are predicted to recur after future invasions. This predictability should be due to the fact that viral growth is dependent on many host factors but human cells do not provide a highly suitable growth condition for viruses invading from nonhumans. Since the time-dependent directional changes could be detected even at a month-level, the information becomes important for designing diagnostics RT-PCR primers for virus detection and therapeutic oligonucleotides with long effectiveness.

1B UCHIDA, Misato ${ }^{1,2}$, NEVO, Eviatar ${ }^{3}$, ASADA, -06 Nobuhiko ${ }^{1}\left({ }^{1}\right.$ Dept of Zool., Faclt. Sci., Okayama Univ. Sci., ${ }^{2}$ Grad. Sch. Biol., Faclt. Sci., Okayama Univ., ${ }^{3}$ Inst. Evol., Faclt. Sci. and Educ., Univ. of Haifa)

Estimation of viability in Drosophila melanogaster at "Evolution Canyon", Israel

The enigma of genetic diversity in natural population has been explored by using evolutionary analysis. The patterns of microscale biodiversity have studied at "Evolution Canyon", Israel. Isofemale Drosophila melanogaster strains, 2-1 strain from the north-facing slope and 6-1 strain from the south-facing slope, and 2,000 egg were prepared in 20 vials. The value of egg-to-adult viability was given in 2-1 strain and 6-1 strain as 0.89 or 0.75 , respectively, showing statistically significant difference. Genetic diversity was higher on the more stressful the south-facing slope than on the milder the north-facing slope. The inter-slope genetic differences were corroborating on the climate selection with viability polygene and in population and stochasticity at the "Evolution Canyon" Israel.
1B MATSUMOTO, Tomotaka ${ }^{1}$, MISHRA, Neha ${ }^{1}$, -07 AKASHI, Hiroshi ${ }^{1}\left({ }^{1}\right.$ Natl. Inst. Genet.)

Testing natural selection in Drosophila intron evolution

Pattern of genome evolution is affected by the interacting effect of multiple factors as natural selection, mutation, recombination and so on. Especially, natural selection acting on base composition like codon usage bias can affect evolution in genome wide level. In this study, we analyzed the variation of base composition of introns in Drosophila melanogaster genome. We found that the base composition is highly variable depending on the size of intron and position within a intron, and the observed heterogeneity of base composition showed strong negative correlation with the genetic divergence. Our result suggests that the evolution of base composition of intron in D. melanogaster can be affected by weak natural selection and therefore, we should be carefully think about using this region as an example of neutral evolution in molecular evolution study.
1B MISHRA, Neha ${ }^{1}$, MATSUMOTO, Tomotaka ${ }^{1}$, -08 AKASHI, Hiroshi ${ }^{1}$ ( ${ }^{1}$ Dept. Genet.)

Transcription and within-gene base composition heterogeneity in D. melanogaster

Evolutionary forces can vary within and across genes and overlooking their variation causes bias in various molecular evolutionary analyses. We used patterns of base composition in D. melanogaster to reveal variation in evolutionary forces within genes. Studying base composition variation within gene can provide insights to the biological processes underlying the evolution of base composition. We found that GC content of introns decreases in the direction of transcription. Association with RNA polymerase binding levels and transcript abundance suggest that transcription-associated mutation or selection govern intron base composition heterogeneity within gene. Such variations in evolutionary forces within genes should be taken into account while using introns or synonymous sites to test models of molecular evolution. 
1B HETTIARACHCHI, Nadeeka Nilmini ${ }^{1}$, SAITOU, -09 Naruya ${ }^{1}\left({ }^{1}\right.$ Natl. Inst. Genet., Mishima, ${ }^{2}$ Natl. Inst. Genet.)

GC content heterogeneity transition of conserved noncoding sequences occurred at the emergence of vertebrates

Conserved noncoding sequences (CNSs) of Eukaryotes are known to be significantly enriched in regulatory sequences. CNSs of diverse lineages follow different patterns in abundance, sequence composition and location. Here we report a thorough analysis of CNSs in diverse groups of Eukaryotes with respect to GC content heterogeneity. We examined 24 fungi, 19 invertebrates, and 12 non-mammalian vertebrates so as to find lineage specific features of CNSs. We found that fungi and invertebrate CNSs are predominantly GC rich as in plants we previously observed, whereas vertebrate CNSs are GC poor. This result suggests that the CNS GC content transition occurred from the ancestral GC rich state of Eukaryotes to GC poor in the vertebrate lineage due to the enrollment of GC poor transcription factor binding sites that are lineage specific. CNS GC content is closely linked with the nucleosome occupancy that determines the location and structural architecture of DNAs.

1B RATNAYAKE, Sangeetha Udani ${ }^{1}$, ENDO, Toshinori ${ }^{1}$, -10 OSADA, Naoki ${ }^{1}\left({ }^{1}\right.$ Hokkaido Univ., ${ }^{2}$ Div. Bioeng. and Bioinform., Hokkaido Univ.)

Revisiting the Relationship between Evolutionary Conservation and Disease Causality: A Case Study of Beta Globin Gene

Predicting the effect of amino acid mutations in genomes plays a vital role in the studies of evolutionary biology and medical genomics. In common practice, the level of evolutionary conservation has been used to infer the biological importance of amino acids, as predicted by the neutral theory of molecular evolution. There is a doubt came up with, whether those consequences of amino acids are actually applicable with accurate disease prediction. In order to answer the question, we investigated the relationship between disease causality and evolutionary conservation in human beta globin gene (HBB), as it has well-known protein structure and disease and non-disease phenotypes are reported for almost all possible amino acid mutations. Unexpectedly, disease causality prediction of $\mathrm{HBB}$ using simple physiochemical grouping performed much better than the conservative predictions. Other than the general concept which determines the evolutionary conservation is important, we are looking for possible reasons why there is a considerable amount of variation of molecular evolution rate within HBB.
1B SABER, Morteza Mahmoudi ${ }^{1,2}$, SAITOU, Naruya ${ }^{1,2,3}$ -11 ( ${ }^{1}$ Dept. Biol. Sci.s, Grad. Sch. Sci., Univ. of Tokyo, ${ }^{2}$ Div. Popul. Genet., Natl. Inst. Genet., ${ }^{3}$ Dept. Genet., Sch. Life Sci., Grad. Univ. Adv. Stud. (SOKENDAI))

Evolution of coding and noncoding genomic sequences shared by humans and great apes

Family Hominidae, which includes humans and great apes, is recognized for unique complex social behavior and intellectual abilities. Despite the increasing genome data, however, the genomic origin of its phenotypic uniqueness has remained elusive. Here we analyzed whole genome sequences of simians in order to find Hominidae-specific genes and highly conserved noncoding sequences (HCNS). We discovered that Down syndrome critical region 4 (DSCR4) is the only experimentally verified gene uniquely present in Hominidae. We showed that DSCR4 has emerged in several steps through LTR/ERV1, LTR/ ERVL retrotransposition, and transversion. Using the genomic distance as neutral evolution threshold, we identified 1,658 Hominidae-specific HCNSs. Polymorphism coverage and derived allele frequency analysis of Hominidae-specific HCNSs showed that these HCNSs are under purifying selection, indicating that they may harbor important functions. Interestingly, many ancestral sequences of the Hominidae-specific HCNSs showed very high evolutionary rates. This suggests that new functions emerged through accelerated evolution, and then purifying selection started to operate to keep these functions.

1B JINAM, Timothy ${ }^{1}$, SAITOU, Naruya ${ }^{1}\left({ }^{1}\right.$ Natl. Inst. -12 Genet., Div. Popul. Genet.)

The genetic history of Negrito populations in Southeast Asia

The Negritos are indigenous human populations currently living in the Philippines, Malay Peninsula and Andaman Islands. They have been of interest to anthropologists due to their unique phenotype which bears resemblance to African pygmies. Using genome-wide SNP analysis, we show their deep divergence from other East Asians and that shared loci among the Negritos are related to some physical features. We also report signals of admixture with archaic humans called Denisovans in the Philippine Negritos. Our results demonstrate the uniqueness of these Negrito populations. 
1C FUKUDA, Kei ${ }^{1}$, YUSA, Kosuke ${ }^{2}$, SHINKAI, Yoichi ${ }^{1}$ -01 ( ${ }^{1}$ Cell. Memory lab., RIKEN, ${ }^{2}$ Wellcome Trust Sanger Inst., Hinxton, Cambridge)

Identification of factors involved in provirus silencing by CRISPR-Cas9 system

Proviruses and endogenous retroviruses (ERVs) are frequently repressed in embryonic stem cells (ESCs). It is known that Histone H3 lysine 9 trimethylation (H3K9me3), mediated by SETDB1, are required for silencing of proviruses and ERVs. However, it remains largely unknown how the silencing state is established and maintained. To advance our understanding of the silencing pathway comprehensively, we developed a powerful high-throughput approach based on a provirus MSCV-GFP reporter and genome-wide genetic screen by CRISPR-Cas9 genome editing technology.
1C NAGAKI, Kiyotaka ${ }^{1}$, YAMAJI, Naoki ${ }^{1}$, MURATA -03 Minoru ${ }^{1}{ }^{1}$ Inst. Plant Sci. and Resources, Okayama Univ.)

Analysis of epigenetic modifications in plant tissues by a novel clearing method

Since investigations of epigenetic status of individual cells in tissues can produce not only epigenetic data in different cell-types but also positional information of the cells, the investigations are important to understand intra- and inter-cell control systems for developments and environmental responses in plants. However, there was no simple way to detect epigenetic modifications of individual cells in plant tissues. In this study, we developed a clearing method for immunohistochemical (ePro-ClearSee) to investigate epigenetic modifications without sectioning. The ePro-ClearSee method could detect methylated histones, acetylated histones, methylated DNA and/or centromeric histone H3 variants in four dicots and five monocots, and made it possible to detect the immunosignals $200 \mu \mathrm{m}$ deep in plant tissues for a short period (10 days-3 weeks). The results suggest this method provide a simple and universal way to investigate epigenetic modifications in widespread plant species.

1C TANIYAMA, Nobuko ${ }^{1}$, KOBAYASHI, Kenta ${ }^{1}$ -04 YAMANE, Junko ${ }^{1}$, MORI, Tomoya ${ }^{1}$, YAMASHITA, Jun K. ${ }^{1}$, FUJIBUCHI, Wataru ${ }^{1}\left({ }^{1}\right.$ Dept. Cell Growth and Differ., Cent. iPS Cell Res. and Application, Kyoto Univ.)

Single-cell methylome analysis of mouse blastocyst

As a zygote divides, it generates different cell types and acquires tissue characteristics. As many epigenetic factors are involved during its process and have been investigated. However, the analyses done by cell population provide averaged information thus masked the feature of each single-cell. Here we performed a single-cell analysis to find out clues to understand mechanisms regulating tissue specificity and conformation. In this study, we focused on DNA methylation, which is known to relate to genomic imprinting and cell identities. Although many DNA methylation analysis methods are reported, only two are applied to single-cell analysis. Considering the genome coverage, analytical flexibility, and costs, we selected the scRRBS (single-cell reduced representation bisulfite sequencing) method. This method contains bisulfite conversion step that causes DNA fragmentations, which decreases sample yield. Therefore, we developed a new version of scRRBS method to increase the analytical range. We used mouse blastocysts and checked the effect of our method. Our method represented more $\mathrm{CpG}$ sites than the original method without chromosome biases. 
1C KUBIURA, Musashi ${ }^{1}$, BOGUTZ, Aaron ${ }^{2}$, KIMURA, -05 Hironobu ${ }^{3}$, TAJIMA, Shoji ${ }^{3}$, LEFEBVRE, Louis ${ }^{2}$, TADA, Masako ${ }^{4}$ ( ${ }^{1}$ Inst. of Regener. Med. Biofunc., Grad. Sch. Med. Sci., Tottori Univ., ${ }^{2}$ Life Sci. Inst., Univ. of British Columbia, Canada, ${ }^{3}$ Inst. Prot. Res., Osaka Univ, ${ }^{4}$ Chromosome Eng. Res. Cent. (CERC), Tottori Univ)

Functional switching of Dnmt1 during mouse ES cells differentiation

Precise control of DNA methylation $(5 \mathrm{mC})$ patterns is essential for mammalian embryonic development. Genome-wide DNA active demethylation occurs in preimplantation embryos. Thereafter, genomes become highly methylated during epiblast formation. Although DNA methyltransferase (Dnmt) 1/3a/3b triple-knockout (TKO) mouse embryonic stem cells (ESCs) can self-renew in the absence of $5 \mathrm{mC}$, they undergo apoptosis under differentiation condition. The major aim of this study was to determine the mechanism underlying an extensive gain of DNA methylation and to examine the physiological function of Dnmts during mouse epiblast formation. We performed in vitro differentiation of epiblast-like cells (EpiLCs) from mouse ESCs lacking maintenance-type Dnmt1 (1KO ESCs) or a de novo-type Dnmt3a and Dnmt3b (DKO ESCs), and TKO ESCs in which a Dnmt1 cDNA transgene was reintroduced (TKO+1). DKO ESCs differentiated into EpiLCs, and levels of $5 \mathrm{mC}$ increased in these cells following differentiation. Furthermore, Dnmt1 restores cell differentiation potency in TKO cells. The results suggest that Dnmt1 possesses a developmentally regulated de novo DNA methylation activity.

1C TAKUNO, Shohei ${ }^{1}$, RAN, Jin-Hua ${ }^{2}$, GAUT, Brandon -06 S. ${ }^{3}$ ( ${ }^{1}$ SOKENDAI, ${ }^{2}$ Chinese Acad. of Sci., ${ }^{3}$ Univ. California)

Evolutionary patterns of genic DNA methylation vary across land plants

Little is known about patterns of genic DNA methylation across the plant kingdom or about the evolutionary processes that shape them. To characterize gene-body methylation within exons, we have gathered single-base resolution methylome data that span the phylogenetic breadth of land plants. We find that a basal land plant, Marchantia polymorpha, lacks any evident signal of genebody methylation, but conifers have high levels of both CG and CHG methylation in expressed genes. To begin to understand the evolutionary forces that shape gene-body methylation, we first tested for correlations in methylation levels across orthologues. Genic CG methylation levels, but not CHG or CHH levels, are correlated across orthologues for species as distantly related as ferns and angiosperms. In contrast, genic CHG methylation correlates with genome size, suggesting that the host epigenetic response to transposable elements also affects genes. Altogether, our data indicate that the evolutionary forces acting on DNA methylation vary substantially across species, genes and methylation contexts.
1C SAITO, Raku ${ }^{1,2}$, TAKASHIMA, Kazuya ${ }^{2}$, TARUTANI, -07 Yoshiaki ${ }^{1,2}$, KAKUTANI, Tetsuji ${ }^{1,2}$ ( ${ }^{1}$ Dept. Genet., SOKENDAI, Sch. Life Sci., SOKENDAI, ${ }^{2}$ Div. Agricltural Genet., Natl. Inst. Genet.)

Specificity and diversity of the transposon-encoded antisilencing factor

Transposons are usually highly methylated and transcriptionally silenced. Arabidopsis transposon VANADL21 family harbors anti-silencing factor named VANC21. When VANC21 gene expressed as transgene, endogenous VANDAL21 sequences are specifically de-methylated and transcriptionally re-activated. In Arabidopsis genome, there are many VANC-like genes. By methylome and transcriptome analysis, it has been suggested that each VANDAL family would have different target specificity. We found that VANC6, VANC-like gene harbored by VANDAL6 family, induces loss of DNA methylation specifically in endogenous VANDAL6 sequences and related families. These highly sequence-specific anti-silencing systems would reduce damage to the host and contribute long-term advantage for the survival of VANDAL families.

1C HOSAKA, Aoi ${ }^{1,2}$, SAITO, Raku ${ }^{1,2}$, TAKASHIMA, -08 Kazuya ${ }^{2}$, SASAKI, Taku ${ }^{2}$, TARUTANI, Yoshiaki ${ }^{1,2}$ KAKUTANI, Tetsuji ${ }^{1,2,3}$ ('1 Div. Agr. Genet., Dept. Genet., SOKENDAI (Grad. Univ. Adv. Stud.), ${ }^{2}$ Dept. Integr. Genet., Natl. Inst. Genet., ${ }^{3}$ Dept. Biol. Sci., Grad. Sch. Sci., The Univ. Tokyo)

Sequence-specific anti-silencing of transposons in Arabidopsis and its rapid evolution

Transposable elements (TEs) are potentially harmful to the host, and they are silenced by epigenetic mechanisms such as DNA methylation. However, some TEs counteract host surveillance. A mobile copy of VANDAL21 TEs in Arabidopsis encodes an antisilencing factor, VANC21. Expression of VANC21 induces loss of DNA methylation specifically on VANDAL21 copies. The bases for the sequence specificity and the evolutionary aspects of the anti-silencing mechanisms are largely known.

Here we report the binding regions of VANC21 protein by ChIPseq and discuss bases for the rapid evolution. 
1C SASAKI, Taku ${ }^{1}$, TARUTANI, Yoshiaki ${ }^{1,2}$, KAKUTANI, -09 Tetsuji $1,2,3$ ( ${ }^{1}$ Natl. Inst. Genet., ${ }^{2}$ SOKENDAI, ${ }^{3}$ Univ. Tokyo)

Inactivation mechanism of VANDAL21 transposons in Arabidopsis thaliana

VANDAL21 is a Mutator transposon which is transcriptionally activated and mobilized in DNA hypomethylation mutant. An autonomous VANDAL21 copy, named Hiun $(\mathrm{Hi})$, encodes three ORFs: VanA, VanB, and VanC. VanA encodes a putative transposase but function of proteins encoded by other ORFs is unknown. When VanC sequence is induced as transgene, endogenous $H i$ and VANDAL21 transposons are specifically reactivated. Interestingly, if the VanC transgene was segregate apart in the next generation, VANDAL21 transposons are inactivated and re-silenced already in that generation. Here we show that this resilencing of $H i$ depends on RNAi machineries. Although the RNAi machinery is not required for keeping $H i$ silent in wild type, RNAi seems to function efficiently for the de novo silencing.

1C TAIKO, To ${ }^{1}$, TARUTANI, Yoshiaki ${ }^{2}, \mathrm{KATO}_{\mathrm{Kae}}{ }^{2}$, -10 INAGAKI, Soichi ${ }^{2}$, ITO, Tasuku ${ }^{2}$, TAKAHASHI, Mayumi $^{2,3}$, TOYODA, Atsushi ${ }^{2}$, FUJIYAMA, Asao ${ }^{2}$, VINCENT, Colot ${ }^{4}$, KAKUTANI, Tetsuji ${ }^{1,2}\left({ }^{1} \mathrm{Grad}\right.$. Sch. Sci., Univ. Tokyo, ${ }^{2}$ Natl. Inst. Genet., ${ }^{3}$ Cent. Ecological Res., Kyoto Univ., ${ }^{4}$ Institut de Biologie (IBENS))

Transgenerational establishment of CG and non-CG DNA methylation patterns in Arabidopsis

DNA methylation is important for silencing transposable elements (TEs) in eukaryotes. In plant genomes, TEs are heavily methylated in both CG and non-CG contexts. A central question still unanswered is how the TE-specific DNA methylation is established. Through genetic and epigenomic analyses, here we examined transgenerational de novo methylation of TEs after loss of methylation in CG or non-CG context. RNAi-dependent de novo methylation of TEs proceed efficiently for both CG and nonCG contexts when DNA methylation of the other context remains. In addition, CG methylation directs precise and efficient recovery of non-CG methylateon in an RNAi-independent manner.
1C INAGAKI, Soichi ${ }^{1}$, TAKAHASHI, Mayumi ${ }^{1}$, -11 KAKUTANI, Tetsuji ${ }^{1,2}$ ( ${ }^{1}$ Dept. Integr. Genet., Natl. Inst. Genet., ${ }^{2}$ Grad. Sch. Sci., The Univ of Tokyo)

Dynamics of gene-body histone modifications mediates heterochromatin silencing in Arabidopsis

Transposons are silenced by histone H3 lysine 9 methylation (H3K9me2) and DNA methylation in many eukaryotes. While it is widely accepted that H3K9me2 and DNA methylation around promoter silence the transcription, many silent transposons have these marks in their transcribed regions (body) and it's function is unknown. We are using an Arabidopsis mutant that accumulates H3K9me2 ectopically within bodies of transcribed genes and shows developmental abnormalities to clarify the roles of gene body chromatin modifications. Through genetic screenings, we have found a factor working downstream of the body H3K9me2.

1C KAJITANI, Takuya ${ }^{1}$, KATO, Hiroaki ${ }^{2}$, CHIKASHIGE -12 Yuji ${ }^{3}$, KIMURA, Hiroshi ${ }^{4}$, OHKAWA, Yasuyuki ${ }^{5}$, HERAND, Damien ${ }^{6}$, MURAKAMI, Yota ${ }^{1}\left({ }^{1} \mathrm{Grad}\right.$. Sch. Sci., Hokkaido Univ., ${ }^{2}$ Grad. Sch. Med. Res., Shimane Univ., ${ }^{3}$ Advanced ICT Res. Inst., Natl. Inst. Inform. and Commu. Tech., ${ }^{4}$ Grad. Sch. BioSci. and BioTech., Tokyo Inst. Tech., ${ }^{5}$ Med. Inst. Bioregulation, Kyushu Univ., ${ }^{6}$ Namur Adv. Res. Coll. (NARC), Namur Univ.)

Phosphorylation at Ser7 of RNA polymerase II CTD limits transcription speed and transcription elongation

C-terminal domain(CTD) of RNA polymerase II(RNAPII) is highly phosphorylated in vivo. Phosphorylation at Ser2 and Ser5 of CTD is coupled with co-transcriptional events, such as activating histone modifications and RNA processing. However, the intimate role of phosphorylation at Ser7 has remained elusive.

We found that non-phospho acceptor alanine substitution mutation (ctdS7A) in fission yeast causes hyperphosphorylation at Ser2 and Ser5 across euchromatic and heterochromatic regions, suggesting hyperactivation of RNAPII. The hyperactivated RNAPII in ctdS7A is likely to transcribe rapidly, inferring that phosphorylation at Ser7 acts as a decelerator of transcription. The rapid transcription is facilitated by Paf1.

In addition, we found that the rapid transcription in ctdS7A across heterochromatin leads to be defective in RNAi-dependent heterochromatin formation. In ctdS7A mutant, Argonaute complex could not target to the nascent transcripts. The defects were suppressed in ctdS7Apaf $1 \Delta \mathrm{C}$ double mutant. These results indicated that at heterochromatic regions, the attenuation facilitated by Ser7 is prerequisite for exertion of RNAi-dependent heterochromatin formation. 
1D KIDERA, Kaho ${ }^{1}$, ODAJIMA, Takuya ${ }^{1}$, TONE, -01 Takahiro $^{1}$, KANESAKI, Tomo ${ }^{2}$, YOSHIKAWA, Hirofumi ${ }^{3}$, MAKINO, Osamu ${ }^{1}\left({ }^{1}\right.$ Fac. Sci. and Tech., Sophia Univ., ${ }^{2}$ NGRC, Tokyo Univ. Agr., ${ }^{3}$ Dept. BioSci., Tokyo Univ. Agr.)

Characterization of the $r s i V$ mutations which affect the growth of subtilis phage $\phi 29$

Because only two host factors, the products of $m r e B$ and $r p o A$, have been reported to be essential for the growth of Bacillus subtilis phage 29, we searched for other host factors by the analysis of Bacillus subtilis spontaneous mutants which affect the growth of 29. After several screening steps, we sequenced the genome of 5 candidates and found out 2 clones which had mutations in $r s i V$ (one was 4 base deletion mutant and another was single nucleotide substitution mutant). Because $r s i V$ is an anti- factor of SigV, we assumed that the increase of SigV regulon expression inhibited the growth of 29 .
Genetic analysis of regulation mechanisms for SigM and its cognate anti-sigma factor, YhdL and YhdK, in Bacillus subtilis

In Bacillus subtilis, a Gram-positive sporulating soil bacterium, possesses various kinds of stress response sigma factors. SigM is strongly involved in response to cell envelope stress. SigM activity is regulated negatively with anti-sigma factor, YhdL and YhdK. Both of YhdL and YhdK proteins are predicted to be membrane protein and the $\mathrm{N}$ terminal peptide of $\mathrm{YhdL}$ is thought to bind to SigM, though their molecular mechanism of regulation is not elucidated precisely. The C-terminal portion of YhdL was gradually deleted. All the deletion leaded to the loss of anti-sigma function. The fusion of GFP and the N-terminal domain of YhdL, which is the putative sigma-binding domain, might lead to the loss of YhdL function. A merodiploid strain, which possessed the C-ter-deleted YhdL and GFP-YhdL fusion, was constructed. This strain restored anti-SigM activity, suggesting that both abortive peptides formed a dimer and compensated lost function (N-ter; sigma-binding and C-ter; signal receiving) with each other. We also revealed that so-called transcriptional coupling was required for efficient expression of YhdL and YhdK. Suppressor mutations of YhdK deletion were obtained and analyzed.
1D YOSHIDA, Saki ${ }^{1}$, YOSHIKAWA, Hirofumi ${ }^{2}$, ASAI, -03 Kei ${ }^{1}\left({ }^{1}\right.$ Dept. Biochem. Mol. Biol., Grad. Sch. Sci. Eng., Saitama Univ., ${ }^{2}$ Dept. BioSci, Grad. Sch. Agr., Tokyo Univ. Agr.)

Research of the representative phenotype and the responsible gene in Bacillus subtilis SigA only strain

Sigma factors, which are involved in the initiation of transcription in bacteria, are one of components of RNA polymerase holoenzyme. Bacillus subtilis possesses 19 sigma factors, and function of alternative sigma factors have been studied mainly from the view of stress response and spore development. In our former studies, these alternative sigma factors have been inactivated simultaneously and finally the strain, SigA only, in which only one essential sigma factor, SigA, functioned, was obtained. The colonies of the strain, SigA only, got transparent and number of the viable cells in colonies decreased rapidly after growing on LB agar plate. Currently, we have succeeded to acquire the suppressor strains, which restored rapid decrease in the number of the viable cells. From the whole genome sequence analysis of these suppressor strains, we tried to find factors, which are responsible for suppressor phenotype. As a result, it was found that defects in rpsU encoding the $30 \mathrm{~S}$ ribosomal protein, S21, are closely related to suppressor phenotype. We are still elucidating the effect of rpsU deletion.

1D SHIMIZU, Yoko ${ }^{1}$, YOSIKAWA, Hirofumi ${ }^{2}$, ASAI, $\mathrm{Kei}^{1}$ -04 ( ${ }^{1}$ Regul. Network of Cellular Mol., Mol. Biol., Faclt. Sci., Saitama Univ., ${ }^{2}$ Faclt. Appl. Bio-Sci., Tokyo Univ. Agr.)

Expression analysis of lysis phenomenon elucidation and SigI by Bacillus subtilis SigI destruction

SigI is an alternative sigma factor that is supposed to involve in heat stress response. We found that the sigI mutant strain was lysed at stationary phase. We thought that SigI might play a role in regulating maintenance of viability of the cells at stationary phase. We presumed that some kinds of signal substances are involved in this lysis phenomenon, and performed the co-culture experiment using the wild-type and the sigI mutant strain. However, the sigI mutant strain was lysed and the wild type strain was not regardless of each other. Therefore, lysis phenomenon was supposed not to be due to induction with the lysis-inducing factors or cell-cell communication. We constructed the strain harboring PsigI-GFP fusion and monitored fluorescence emitted from the individual cells at stationary phase. Flow cytometric analysis showed that PsigI was activated in limited number of the cells (around 4\%). Cell-staining analysis, by which the live cells could be distinguished from the dead cells under the fluorescence microscope, shows that the number of dead cells increased in the sigI mutant strain. 
1D SEKI, Takahiro ${ }^{1}$, MATSUOKA, Satoshi ${ }^{1}$, -05 MATSUMOTO, Kouji ${ }^{1}$, HARA, Hiroshi ${ }^{1}\left({ }^{1}\right.$ Grad. Sch. Sci. Eng., Saitama Univ.)

Analysis of the activation mechanism of an extracytoplasmic function $\sigma$ factor $\sigma \mathrm{V}$ in the Bacillus subtilis mutant cells lacking glucolipids by using chimera-antisigma factors

The Bacillus subtilis cells lacking glucolipids by disruption of the $u g t P$ gene show activation of extracytoplasmic function (ECF) $\sigma$ factor $\sigma^{\mathrm{V}} \cdot \sigma^{\mathrm{V}}$ is regulated directly by transmembrane anti- $\sigma^{\mathrm{V}}$ RsiV. The $\sigma^{\mathrm{V}}$ is activated specifically by lysozyme. When $B$. subtilis cells are challenged by lysozyme, RsiV is cleaved by regulated intramembrane proteolysis (RIP). However, we did not observe the proteolysis of RsiV in the $\Delta u g t P$ cells. This result suggested that the lack of glucolipids activated the $\sigma^{\mathrm{V}}$ by another mechanism. To elucidate the mechanism, we constructed the two chimera anti- $\sigma, \operatorname{RsiV}_{\mathrm{N}}-\mathrm{RsiW}_{\mathrm{TMC}}$ and $\mathrm{RsiV}_{\mathrm{NTM}}-\mathrm{RsiW}_{\mathrm{C}}$, by swapping the intracellular $\mathrm{N}$-terminal domain or extracellular $\mathrm{C}$ terminal domain of RsiV and those of RsiW. RsiW is anti- $\sigma^{\mathrm{W}}$, and $\sigma^{\mathrm{W}}$ does not respond to the lack of glucolipids. These chimera anti- $\sigma$ were introduced into the $\Delta r s i V$ and the $\Delta r s i V \Delta u g t P$ cells, and the $\sigma^{\mathrm{V}}$ activity was measured by monitoring the $\beta$ galactosidase activity expressed from PsigV-lacZ. The $\sigma^{\mathrm{V}}$ activity decreased to the wild-type level by expression of RsiVNRsiWTMC or RsiVNTM-RsiWC in the $\Delta r s i V \Delta u g t P$ cells. This result suggested that $\mathrm{C}$-terminal domain of $\mathrm{RsiV}$ sensed the lack of glucolipids.

1D MATSUOKA, Satoshi ${ }^{1}$, MATSUMOTO, Kouji ${ }^{1}$, ASAI, -06 Kei ${ }^{1}$, HARA, Hiroshi ${ }^{1}$ ( ${ }^{1}$ Grad. Sch. Sci. and Eng., Saitama Univ.)

Molecular mechanism of regulation of two component system WalKR by glucolipids in Bacillus subtilis

Bacillus subtilis cell membranes contain $10 \%$ glucolipids. They are synthesized by UgtP processively. ugtP mutants are bent and distended in log phase. This indicates that glucolipids are important for the maintenance of cell morphology. In this study we focused on WalKR two component system and SigI, which both are involved in cell wall maintenance. We constructed a series of sigI promoter which has substitution and/or deletion in its cis element sequences with $l a c Z$ transcriptional fusion. Not only SigI but also WalR was activated by the depletion of glucolipids resulting from the lacZ assays. Then we constructed a B. sbutilis starain which has walR-hisx 12 in its chromosome to detect the degree of phosphorylation. The phosphorylation of WalR was accelerated by the depletion of glucolipid using the phos-tag method. These suggest that glucolipids may be required for SigI and WalKR functions.
1D IZAWA, Tomoko ${ }^{1}$, MATSUMOTO, Kouji ${ }^{1}$, -07 MATSUOKA, Satoshi ${ }^{1}$, HARA, Hiroshi ${ }^{1}\left({ }^{1}\right.$ Div. Life Sci., Grad. Sch. Sci. Eng., Saitama Univ.)

Function of E.coli RcsF in activation of Rcs signal transduction system

The Rcs signal transduction system of E.coli is one of the twocomponent regulatory systems and regulates the expression of many envelope-related genes. These genes respond to the cell surface stresses and implicated in biofilm formation. For activating this system, an outer membrane lipoprotein RcsF is essential. RcsF penetrates the outer membrane through the ?barrel lumen of OMP and anchors in outer leaflet of the outer membrane by the $\mathrm{N}$-terminal acyl chains. A part of $\mathrm{N}$ terminus of $\mathrm{RcsF}$ is exposed on the cell surface (Konovalova et al., 2014). In this study, we focused on the localization of RcsF in various conditions that activate the Rcs system. We constructed RcsF with the FLAG tag at various positions. We transformed these RcsFs to the strains that activate the Rcs system and detected the domains of these RcsFs that were exposed to the surface. When the Rcs system was activated by gene defects or osmolar stress, RcsF seemed to be kept anchored in the outer leflet of the outer membrane and to change the structure to approach the inner membrane. We supposed that the C-terminal portion of surfaceexposed domain of RcsF slid into the lumen of OMP.

1D SATO, Takatsugu ${ }^{1}$, TAKANO, Akira ${ }^{1}$, MATSUOKA -08 Satoshi ${ }^{1}$, MATSUMOTO, Kouji ${ }^{1}$, HARA, Hiroshi ${ }^{1}$ $\left({ }^{1}\right.$ Dept. Biochem. Mol. Biol., Grad. Sch. Sci. Eng., Saitama Univ.)

Visualization of protein-protein interactions among Rcs components in Escherichia coli by Periplasmic BiFC

E. coli Rcs system is one of the two-component systems and responds to various envelope stresses. It regulates enveloperelated genes including the genes responsible for colonic acid synthesis required for biofilm formation. The Rcs system has much more components than typical two-component systems. Minor outer membrane lipoprotein RcsF is one of the essential components of the system. We speculated that RcsF could transmit the signal to histidine kinase RcsC in the cytoplasmic membrane directly. However, little is known about detailed transduction mechanism. Here we tried to visualize the direct protein-protein interaction among Rcs components in the periplasmic space by Bimolecular Fluorescence Complementation (BiFC). We constructed experimental system for detection of interaction in the periplasmic space by fusing the sec signal sequence to BiFC fusion proteins. We could detect interaction of $\mathrm{RcsF}$ with the periplasmic domein of RcsC and $\mathrm{YrfF}$ (a negative regulator of the Rcs system) interaction. This result indicates RcsF transmit the signal by interaction with two different inner membrane proteins. We are exploring the effects of these interactions on Rcs activation. 
1D OGAWA, Naoya ${ }^{1}$, HAYASHI, Hiroki ${ }^{1}$, KISHI, -09 Tsutomu ${ }^{1}$ ( ${ }^{1}$ Coll. Eng., Nihon Univ.)

Phosphorylation by Pho85/CDK5 triggers degradation of the transcription factor Swi5 via the $\mathrm{SCF}^{\mathrm{Cdc} 4}$ ubiquitin ligase

Degradation of proteins by the ubiquitin pathway is important for various cellular regulatory mechanisms. The $\mathrm{SCF}^{\mathrm{Cdc} 4}$ complex is the ubiquitin ligase complex that acts as a regulator of cell cycle, signal transduction and transcription. We have previously reported that the $\mathrm{SCF}^{\mathrm{Cdc} 4}$ complex regulates $\mathrm{S}$ phase initiation through degradation of Swi5, the transcription factor required for transcription of SIC1, the S phase Cdk inhibitor. However, the mechanism by which Swi5 is degraded by the $\mathrm{SCF}^{\mathrm{Cdc} 4}$ ubiquitin ligase remains unknown. We repot here that Pho85/CDK5 in involved in the degradation of Swi5. We used Swi5-ST8A, in which S225, S231, S246, 250, S261, S300, T320 and T323 are substituted by alanine residues, and is partially stabilized), to demonstrate this. Swi5-ST8A was much more stabilized in pho85 mutants than in wild type. The result suggests the presence of the phosphorylation site(s) other than the ST8A mutation for the degradation of Swi5. We identified the serine residue, and substitution of this to alanine residue (Swi5-ST9A) stabilized Swi5-ST9A. In addition, we also identified Pcl (Pho Cyclin) subunits required for Swi5-degradation.

1D HORIKE, Shin-ichi ${ }^{1}$, MEGURO, Makiko ${ }^{1}$, NUMATA -10 Saya ${ }^{1,3}$, HONDA, Toshiya ${ }^{1,3}$, OKADA, Gensaku ${ }^{1}$, YOKOYAMA, Shigeru ${ }^{2}$, HIGASHIDA, Haruhiro ${ }^{2}$ $\left({ }^{1}\right.$ Adv. Sci. Res. Cent., Kanazawa Univ., ${ }^{2}$ Res. Cent. Child Mental Dev., Kanazawa Univ., ${ }^{3}$ Sch. Med., Kanazawa Univ.)

Identification of novel distant regulatory element in the OXTR gene locus

Characterized by behavioral and cognitive disturbances of childhood development, autism spectrum disorders (ASD) are currently estimated to affect 1 in 68 children, with a male:female ratio of $4: 1$. The etiology of ASD remains unknown, though it likely involves interactions between multiple genetic and environmental risk factors. To date, only a small percentage of autism cases $(<10 \%)$ have been ascribed to single gene disorders such as Rett syndrome, tuberous sclerosis complex, and fragile $\mathrm{X}$ syndrome. However, the genetic alterations of ASD remain elusive in the majority of cases. Current studies suggested that OXTR and the oxytocin signaling pathway play an important role in the etiology of ASD. In this study, we applied CRISPR/Cas9 genome editing system to generate a deleted allele of the regulatory element of OXTR gene expression. We found that knockout (KO) allele disrupted the expression of OXTR.
1D AMANO, Takanori ${ }^{1}$, SHIROISHI, Toshihiko ${ }^{1}\left({ }^{1}\right.$ Natl. -11 Inst. Genet.)

Cis-regulatory polymorphism of $S h h$ is involved in variation of shape and size of the mouse stomach

Polymorphism in cis-regulatory elements can cause differentiation of gene regulation, and plays a critical role in divergence of quantitative traits. Stomach is one of the organs that are morphologically divergent among vertebrates, implying interspecies variation of expression level of developmental genes. Stomach development may be a good model to know how cisregulatory variations generate morphological diversity.

Shh is expressed in endodermal epithelia during development, and contributes to cell proliferation. Here we found that a $1.7 \mathrm{~Kb}$ region located $100 \mathrm{~Kb}$ upstream of Shh contains a stomach enhancer, and named it SLGE.

SLGE, which is evolutionally low conserved and found only in rodents, has several SNPs and Indels when comparing C57BL/6 (B6) with MSM. Our luciferase assay revealed that MSM SLGE has higher transcriptional activity than B6 SLGE does. Moreover, high expression of $S h h$ was observed in the embryonic stomach of MSM as compared to B6. High regulatory activity of MSM SLGE is consistent with the fact that MSM has a relatively large stomach. Taken together, cis-regulatory variation in SLGE may play a role in size control of the stomach in rodents. 
1E KOKUBA, Shun ${ }^{1}$, ETO, Satoshi ${ }^{1}$, ARAKI, Miyuki ${ }^{1}$, -01 MORITA, Ayaka ${ }^{1}$, KIMACHI, Yoko ${ }^{1}$, YAMAMURA, Kenichi $^{1}$, YOSHINOBU, Kumiko ${ }^{1}$, ARAKI, Kimi ${ }^{1}$, ARAKI, Masatake ${ }^{1}{ }^{1}$ Inst. Resource Dev. and Anal., Kumamoto Univ.)

Establishment and analysis of Cre-driver mouse line (Administration of Tamoxifen for Cre-mERT2)

We have developed Database for the Exchangeable Gene Trap Clones (EGTC) (http://egtc.jp). In our system, reporter beta-geo gene can be exchanged into any other DNA of interest through Cre-mediated recombination. We have produced more than 300 Cre driver ES cell lines using EGTC ES cells. Among them, more than 100 mouse lines could be established. Then we did mating of Cre driver mice with ROSA26R mice, and performed X-gal staining of ROSA26R mice with Cre driver allele.

In this study, we used tamoxifen-inducible CremERT2 mouse lines. We could demonstrate that administration method of Tamoxifen added to dry non-pelleted feed is better than general administration method of Tamoxifen by intraperitoneal injection, concerning to recombinase efficiency, work efficiency and animal welfare.
$1 E$ SHIBUYA, Hirotoshi ${ }^{1}$, ICHIMURA, Kaoru ${ }^{1}$, MAENO, -03 Akiteru ${ }^{2}$, UEDA, Akihiro ${ }^{1}$, TAMURA, Masaru ${ }^{3}$ WAKANA, Shigeharu ${ }^{3}$, SHIROISHI, Toshihiko ${ }^{2}$ YAMAMOTO, Akitsugu ${ }^{1}$, YAMAMOTO, Hiroaki ${ }^{1}$ $\left({ }^{1} \mathrm{Grad}\right.$. Sch. Biosci., Nagahama Inst. Bio-Sci. Tech., ${ }^{2}$ Mammalian Genet. Lab., Natl. Inst. Genet., ${ }^{3}$ Riken $\mathrm{BRC)}$

Mitf affects the development of mouse eye-balls

Melanocytes are derived from the vertebrate embryo-specific neural crest. Melanocytes localize in various organs, including not only the skin but also extracutaneous locations such as the choroid of eye, inner ear, heart, etc. We have been interested in the functional differentiation (evolution) of these pigment cells. To approach this question, we need to know what these extracutaneous melanocytes are doing in such sun-protected habitats, still expressing their icon function, i.e., synthesizing melanin. We first focused on melanocytes in the eye that localize in its melanin rich layer called the "uvea" consisting of choroid, iris, ciliary body. They may still be illuminated by the transmitted light through the retinal pigment epithelium (RPE), another type of melanin pigment cells derived from the outer layer of the optic cup. RPE is believed to mostly absorb sunlight. We used a mouse Mitf mutant allele, Mit $f^{m i-b w}$. Because Mit $f^{m i-b w}$ homozygous mice can't develop melanocytes but RPE, they show a black-eyed white phenotype. Our observation suggests that the choroidal melanocytes may contribute to support morphogenesis and/or maintenance of the normal vasculature structure.

1E KATAOKA, Taro ${ }^{1,2}$, TAMURA, Masaru ${ }^{3}$, MAENO, -04 Akiteru ${ }^{2}$, SHIROISHI, Toshihiko ${ }^{2}{ }^{1}$ Shizuoka Med. Res. Cent. disaster, Juntendo Univ., ${ }^{2}$ Mamm. Genet. Lab., Genet. Strains Res. Cent., Natl. Inst. Genet., ${ }^{3}$ Technol. and Dev. Team for Mouse Phenotype Anal., RIKEN BioRes. Cent.)

Exploration of Genetic Factors Controlling Bone Metabolism of Mouse

Bone volume is controlled by the balance between osteoblastic bone formation and osteoclastic bone resorption, called bone remodeling. To elucidate genetic mechanisms of bone remodeling during early growth phase, we dissected mouse genome by using B6-MSM consomic mouse strains. We have reported that there are multiple QTLs for regulating bone volume on mouse chromosome 15 .

One of those QTLs, it have four candidate genes and we generated each candidate gene mutant mouse strains by CRIPR/Cas9 system. This time, we announce the result of bonerelated phenotype screening of these mutant strains and its functions as a novel gene for regulating bone volume. 
1E SAGAI, Tomoko ${ }^{1}$, AMANO, Takanori ${ }^{1}$, MAENO, -05 Akiteru ${ }^{1}$, MIZUSHINA, Youichi ${ }^{1}$, NAKAZAWA, Hiromi $^{1}$, SHIROISHI, Toshihiko ${ }^{1}$ ( ${ }^{1}$ Mamm. Genet. Lab., Natl. Inst. Genet.)

Dosage effect of two Shh enhancers for control of tooth number in mouse

In mammalian evolution, the tooth number has been reduced from ancestral form. In mouse, adult has only one incisor and three molars in each jaw quadrant, but embryos develop many vestigial teeth between incisor and the first molar. Notably, mutations of several genes implicated in Wnt, Fgf, Bmp and Shh signaling pathways cause a supernumerary tooth juxtapose to the first molar. To elucidate the precise control for normal dentition, identification of the regulatory mechanism required for these signaling pathways is crucial. Here, we found that elimination of an epithelium-specific $S h h$ enhancer causes such a supernumerary teeth at low frequency. Furthermore, incidence of the abnormal tooth was vastly elevated in the compound heterozygotes between this knockout (KO) allele and Shh coding $\mathrm{KO}$ allele or in the double KO alleles with another epitheliumspecific $S h h$ enhancer. It suggests that dose reduction of the Shh signaling in the dental epithelium is a direct cause of the tooth defect, and two epithelium specific $S h h$ enhancers contribute to dose-dependent control of tooth number. Finally, the sequence comparison identified regulatory cis-motifs shared by the two enhancers.

$1 E$ FURUHATA, Riki ${ }^{1}$, NAKAHARA, $\mathrm{Mai}^{1}$, ITOU, -06 Haruka ${ }^{1}$, ARAKI, Masatake ${ }^{2}$, YOSHINOBU Kumiko ${ }^{2}$, YAMAMURA, Ken-ichi ${ }^{3}$, ARAKI, Kimi ${ }^{1}$ $\left({ }^{1}\right.$ Div. Dev. Genet., Inst. Resource Dev. and Anal., Kumamoto Univ., ${ }^{2}$ Div. Bioinfomatics, Inst. Resource Dev. and Anal., Kumamoto Univ., ${ }^{3}$ Yamamura Project Lab., Inst. Resource Dev. and Anal., Kumamoto Univ.)

Correlated expression of LincRNA-p21 and p53 in the LincRNA-p21 gene trap mouse line

The LincRNA-p21 gene was recently identified as a long intergenic non-coding RNA (lincRNA) regulated by p53. Many interesting functions of the LincRNA-p21 have been reported, but most of studies were performed using culture cells, therefore, the in vivo function has remained unclear. We have established LincRNA-p21 gene trap mouse line (LincRNA-p21gt) and analyzed the expression pattern and phenotypes. The trap vector was inserted in the intron at $12 \mathrm{~kb}$ downstream of the 1st exon, and the fusion transcript of the 1st exon and the LacZ gene was produced. To examine the expression pattern of the LincRNA-p21 in adult mice, $\mathrm{qRT}$-PCR and X-gal staining were performed. The LincRNA-p21 was expressed at low level in almost all tissues examined, and relatively higher expression was observed in the cerebellum, pancreas and white adipose tissue. In order to know whether the LincRNA-p21 is induced by p53 in vivo, we crossed LincRNA-p21 gene trap line to p53-deficient (p53-/-) mice. In LincRNA-p21gt/+; p53-/- mice, X-gal staining in the cerebellum, pancreas and WAT completely disappeared, indicating that the LincRNA-p21 is regulated by p53 in vivo.
$1 E$ SEKI, Ryohei ${ }^{1,2}$, LI, $\mathrm{Cai}^{3}$, MATSUBARA, Haruka ${ }^{2}$, -07 KONDO, $\mathrm{Mao}^{2}$, SATO, Tomohiko ${ }^{2}$, EGAWA, Shiro ${ }^{2}$, HAYASHI, Shinichi ${ }^{2,4}$, SAITO, Daisuke ${ }^{2}$, IRIE, Naoki ${ }^{5}$, ZHANG, Guojie ${ }^{3}$, TAMURA, Koji ${ }^{2}$, SHIROISHI, Toshihiko ${ }^{1}{ }^{1}$ Genet. Strains Res. Cent., Natl. Inst. Genet., ${ }^{2}$ Grad. Sch. life Sci., Tohoku Univ., ${ }^{3}$ BGI-Shenzhen, ${ }^{4}$ Dept. Genet., Cell Biol. and Dev., Univ. of Minnesota, ${ }^{5}$ Grad. Sch. Sci., Univ. Tokyo)

Roles of genomic sequences specifically conserved in Aves on macroevolution of avian-specific morphological features

Birds are characterized by unique morphological traits such as the wing and feather. Although their developmental mechanisms have been studied, the genomic background underlying the emergence of the traits remains unclear. We hypothesized that avian-specific highly conserved elements (ASHCEs) should have crucial functions and identified them by comparing whole genomes of 48 birds and 9 outgroup vertebrates. We found that most ASHCEs are located in non-coding regions, suggesting that they play roles in regulating expression of the genes that they are associated with. To identify such genes, we performed multispecies screening of ASHCEs-associated genes and found that Sim1 was specifically expressed in the region where flight feathers develop in the chicken embryo. Furthermore, reporter assay analyses revealed that the Sim1-associated ASHCE had enhancer activity in the endogenous expression domain of Sim1 in the chicken wing. Interestingly, similar reporter activity was also observed in the mouse forelimb. Taken together, our results suggest that acquisition of novel cis-regulatory elements led to avian-specific gene expression, which resulted in creation of avian-specific traits.

$1 E$ MINEI, Ryuhei ${ }^{1}$, MORI, Tsukasa ${ }^{2}$, OGURA, Atsushi ${ }^{1}$ -08 ( ${ }^{1}$ Nagahama Inst. Bio-Sci. Tech., ${ }^{2}$ Dept. Marine Sci. and Resources, Nihon Univ. Coll. of Bioresour. Sci.)

Brain transcriptome analysis of predator-specific stress response in Rana pirica

The tadpole of Ezo Brown Frog, Rana pirica, shows predatorspecific morphological changes. Larvae of Dragonfly, Aeshna nigroflava, induces heightened tail depth, whereas those of salamander, Hynobius retardatus, induces a bulgy morphology. Although, we identified genes related to their predator-specific changes, it is not understood how $\mathrm{R}$. pirica distinguish $\mathrm{A}$. nigroflava and $\mathrm{H}$. retardatus, and how morphological changes occur. We hypothesized that the recognition of predators in the brain is a trigger for this predator-specific morphological changes. Therefore, we analyzed the brain transcriptome by RNA-sequencing to compare the tadpoles of R. pirica under the various predator stresses. As a result, we identified several gene clusters corresponding to these predator stresses, which are involved in neural network changing and hormone secretion in the brain. Especially this hormone, secreted from the pituitary gland, is thought to be important cue to induce $\mathrm{H}$. retardatus specific morphological change, bulgy morphology. We thus found that the brain is involved in this predator-specific morphological defense. 
$1 E$ TAKAHASHI, Toshiki ${ }^{1}$, MYOSYO, Taijun ${ }^{2}$, SATO, -09 Tadashi ${ }^{1}$, SAKAIZUMI, Mitsuru ${ }^{1}\left({ }^{1}\right.$ Grad. Sch. Sci. and Tech., Univ. of Niigata, ${ }^{2}$ Environ. Life Sci., Shizuoka Prefectural Univ.)

Genetic analysis of a XX male in wild medakas from Kyotango

We established an XX strain from a Dmy-negative(XX) male found in a wild medaka(O.sakaizumii) population at Kohyama, Kumihama, Kyotango. XX males appeared in the $\mathrm{F} 1$ progeny between an XX male of this strain and XX females of an inbred strain, Hd-rR(O.latipes), suggesting that $\mathrm{XX}$ maleness is a dominant trait. We obtained backcross progeny from the F1 males and Hd-rR females, and mapped the causal region to $0 \mathrm{Mb} 1.1 \mathrm{Mb}$ on linkage group 9. Breakpoint analyses narrowed the causal region to $183 \mathrm{~kb} \sim 540 \mathrm{~kb}$. This region included Dmrt1, a downstream gene of Dmy. Amino acid sequences of Dmrt1 derived from this $\mathrm{XX}$ strain and an inbred strain, HNI-?(O. sakaizumii), were the same. These results suggested that Dmrt1 is a prime candidate gene responsible for XX maleness in this strain, and that XX maleness could be caused by overexpression of Dmrt1.

\section{$1 E \quad$ MURAI, Koji ${ }^{1}$ ( ${ }^{1}$ Dept. BioSci., Fukui Pref. Univ.)} $-11$

Cytoplasmic homeosis: pistillody, homeotic transformation of stamens into pistil-like structures, in alloplasmic wheat lines

The homeotic transformation of stamens into pistil-like structures (pistillody) has been observed in cytoplasmic substitution (alloplasmic) lines of bread wheat (Triticum aestivum) that carry the cytoplasm of wild relative Aegilops crassa. We showed previously that the mitochondrial gene orf260 somehow altered the expression patterns of nuclear class B MADS-box genes and $T a D L$ gene, which specify stamens and pistils respectively, to induce pistillody. Bread wheat cultivar Chinese Spring has a fertility restoring gene $R f d 1$ on chromosome 7BL and did not show pistillody with Ae. crassa cytoplasm. The ditelo-somic 7BS line which lacks $R f d 1$ showed pistillody, whereas the monotelodisomic 7BS line which has one $R f d 1$ exhibited pistillody in the two stamens out of three in each floret. The dosage effect of $R f d 1$ on pistillody induction and transmission of $R f d 1$ to next generation would be discussed.
$1 E$ TANAKA, Wakana ${ }^{1}$, HIRANO, Hiro-Yuki ${ }^{1}\left({ }^{1}\right.$ Dept. -10 Biol. Sci.s, Grad. Sch. Sci., Univ. of Tokyo)

Functional analysis of two related genes involved in the axillary meristem formation in rice

Plant development depends on the function of the meristem. Aerial parts of the plant body are built by the activities of two distinct meristems, the shoot apical meristem (SAM) and the axillary meristem (AM). Although a number of studies concerning the SAM formation have been reported, studies about the AM formation are insufficient.

We found that, unlike in Arabidopsis, the AM is formed in a stepwise manner in rice. Analysis of the tillers absent1 (tab1) mutant indicated that $T A B 1$ is required for the maintenance of the pre-meristem zone (PMZ), the initial stage of this developmental process. TAB1 was expressed in the PMZ, although its expression disappeared in the AM after its establishment. By contrast, a related WOX gene, WOX4 was expressed in the established AM but not in the PMZ. Since WOX4 is required for the maintenance of the SAM, these results suggest that two related WOX genes are sequentially expressed to maintain the undifferentiated state of cells during the AM formation.

Lastly, to reveal the difference in the function between $T A B 1$ and WOX4, we generated transgenic rices expressing WOX4 under the control of the $T A B 1$ promoter and analyzed their phenotypes.
$1 E$ SHIBUTA, Mio ${ }^{1}$ AYAKO, Watanabe $^{1}$, ABE, -12 Mitsutomo ${ }^{1}\left({ }^{1}\right.$ Dept. Biol. Sci.s, Grad. Sch. Sci., Univ. of Tokyo)

FE, Myb-related protein, promotes photoperiodic flowering in Arabidopsis

The timely flowering is one of important keys in reproductive success.

One of major component of florigen turned out to be a FT protein, and the molecular mechanism of $F T$ transcription in leaves plays an important role as an accumulation point of various information. In Arabidopsis, CONSTANS (CO) is an essential regulator of photoperiod-dependent flowering; nevertheless over-expression of $C O$ failed to induce ectopic expression of $F T$. So we aimed to identify the other $F T$ transcription activator. A classical floral regulator, $\mathrm{FE}$, is a Myb transcription factor. A single amino acid substitution mutant, $f e-1$, exhibited late-flowering phenotype under long day conditions, and $C O$ failed to activate $F T$ transcription in $f e-1$ background. Conditional over-induction of $F E$ also activated $F T$ transcription depending on $\mathrm{CO}$ activity. Interestingly, over-expression of both $F E$ and $C O$ synergistically induced $F T$ transcription. These results suggest that $\mathrm{FE}$ and $\mathrm{CO}$ interact each other and regulate the spatiotemporal expression of $F T$. 
2A HUA, Liu ${ }^{1}$, ONO, Seijiro ${ }^{1}$, TSUDA, Katsutoshi ${ }^{1,2}$, -01 HIRATSUKA, Rie ${ }^{3}$, FUKAI, Eigo ${ }^{4}$, OHTANI, Misato ${ }^{5}$, DEMURA, Taku ${ }^{5}$, NONOMURA, Kenichi ${ }^{1,2}\left({ }^{1}\right.$ Exp. Farm, Natl. Inst. Genet., ${ }^{2}$ Grad. Sch. Life Sci., SOKENDAI/Grad. Univ. Adv. Stud., ${ }^{3}$ Div. Biol., Jikei Univ. Sch. Med., ${ }^{4}$ Faclt. Agr., Niigata Univ.rsity, ${ }^{5}$ Grad. Sch. Biol. Sci., Nara Inst. Sci. Tech.)

Germline-subcellular localization of rice Argonaute protein MEL1, essential for faithful meiosis progression

Argonaute (AGO) is a protein that associates a single-strand 2030 nucleotides (nt) small RNA as a guide molecule, and functions in transcriptional (TGS) or posttranscriptional gene silencing (PTGS). A rice protein MEL1 is an AGO protein that is exclusively expressed in male and female germ cells before meiosis. MEL1 associates preferentially to 21- and 24-nt siRNAs abundantly expressed in rice anthers. In this study, to ask the molecular function of MEL1, we conducted immunofluorescent staining of rice germ cells, immuno-electron microscopic observations (immuno-EM), and co-immunoprecipitation (co-IP)/ LC$\mathrm{MS} / \mathrm{MS}$

The co-IP fraction of MEL1 contained a lot of ribosomal subunit proteins, as representing a cytoplasmic role of MEL1 in PTGS. Actually, the immuno-EM suggested that MEL1 could associate ribosomes or rough endoplasmic reticulum. Interestingly, the same fraction also contained histone $\mathrm{H} 2$ and $\mathrm{H} 3$ variants. Taken together with the fact that MEL1-GFP fusion protein could locate at the nucleoplasm at the onset of meiosis, the results suggest that MEL1 plays some important role in TGS in male meiocyte nuclei.

ONO, Seijiro ${ }^{1}$, TSUDA, Katsutoshi ${ }^{1,2}$, TANAKA, -02 Keisuke ${ }^{3}$, SASAKI, Takuji ${ }^{4}$, NONOMURA, Ken-ichi ${ }^{1,2}$ $\left({ }^{1}\right.$ Exp. Farm, Natl. Inst. Genet., ${ }^{2}$ Dept. Life Sci., SOKENDAI/Grad. Univ. Adv. Stud., ${ }^{3}$ NODAI Genome Res. Center, Tokyo Univ. Agr., ${ }^{4}$ NODAI Res. Inst., Tokyo Univ. Agr.)

Production of meiotic phasiRNAs is depended on TTM transcription factor in anther tapetum of rice

It has been poorly understood whether plants have small RNAmediated silencing systems during meiotic events, like as the piRNAs in animals. 24-nucleotides (nt) phased small interfering RNAs (phasiRNAs) are the small RNAs which abundantly expressed in meiotic anthers of grass species, though their function on reproduction events is largely unknown.

In this study, we characterized TTM transcription factor in rice, which specifically expressed in anther tapetum, the somatic nursery cells of male meiocytes, during early meiotic stages. $\mathrm{ttm}$ mutant exhibited meiotic retardation and chromosome decondensation in male meiocytes, while tapetum development itself seemed normal. TTM simultaneously activated the transcription of a number of noncoding precursor loci generating 24-nt phasiRNAs and the $D C L 3 b$ gene, encoding a dicer-like protein essential for the 24-nt phasiRNA processing.

Our results indicated the biogenesis of 24-nt phasiRNAs was depended on TTM transcription factor in meiotic tapetum. We also hypothesize that the tapetum-derived 24-nt phasiRNAs have important function in meiosis progression in adjacent meiocytes.
2A HIGASHIDE, Mika ${ }^{1}$, SHINOHARA, Miki ${ }^{1} \quad\left({ }^{1}\right.$ Lab. -03 Genome and Chromosome Funct., Inst. Prot. Res., Univ. Osaka)

Slx4, multifunctional scaffold protein, functions for negative regulation of $\mathrm{CO}$ formation specific in centromere-proximal region and DSB distribution on entire chromosomes

During meiosis, crossover $(\mathrm{CO})$ recombination between homologous chromosomes ensures proper segregation of homologous chromosome in meiosis I. Thus, at least one CO must be formed on each chromosome. In contrast, excessive formation of $\mathrm{COs,}$ especially centromere-proximal region, can be topologically harmful for chromosome segregation. Then, the number and position of COs must be strictly controlled during meiosis. Meiotic $\mathrm{CO}$ formation is induced from programmed double-strand breaks (DSBs) by Spo11 function which is meiosis-specific topoisomerase-like transesterase. Actually, many of endonucleases are required to generate $\mathrm{CO}$ product in each step: DNA end resection for homology search, resolution of CO intermediates. In fact, many kinds of endonucleases are available to recognize and manage intricate DNA structures in vivo. In this study, we focused on Slx4 which is known as scaffold protein for many of DNA structure-specific endonucleases activity in vegetative cells. Interestingly, we found that Slx4 is involved in negative control for $\mathrm{CO}$ formation in centromere-proximal region and in the distribution for DSB formation independent of CO suppression on centromere-proximal region. Thus, we conclude Slx4 is multifunctional in meiotic recombination.

2A KE, $\mathrm{Li}^{1}$, SHINOHARA, Miki ${ }^{1,2}$ ( ${ }^{1}$ Lab. Genome-04 Chromosome Funct, Inst. Prot. Res., Univ. of Osaka, ${ }^{2}$ Dept. Biol. Sci.s, Grad. Sch. Sci., and Faclt. Sci., Univ. Osaka)

Characterization of PP4 Phosphatase Function During Meiotic Recombination in Budding Yeast

Meiotic recombination is essential of proper segregation of chromosomes during meiosis-I by regulating the formation of Chiasma, the physical connection between homologous chromosomes. The regulation mechanism of meiotic recombination is still unclear because of its complexly. In this study, I focused on PP4, a Mec1-specific phosphatase, to reveal its function in meiotic recombination. By now, I confirmed PP4 is involved in the regulation of meiosis. Firstly, I found PP4 can regulate the dephosphorylation of Hop1. Then, it is revealed that PP4 promotes disassembly of factor from chromosomes, such as Zip1 or Rad51. In future, I want to investigate PP4 function during meiotic recombination so that it can facilitate revealing the mechanism of meiotic recombination. 

ITO, Kentaro ${ }^{1}$, MURAYAMA, Yasuto ${ }^{3}$, TAKAHASHI,
Masayuki $^{2}$, IWASAKI, Hiroshi ${ }^{3}\left({ }^{1}\right.$ Dept. Life Sci., Grad. Sch. BioSci. and BioTech., Tokyo Inst. Tech., ${ }^{2}$ School and Grad. Sch. BioSci. and BioTech., Tokyo Inst. Tech., ${ }^{3}$ Cell Biol Unit, Inst. innovative Res., Tokyo Inst. Tech.)

Real-time analysis of DNA strand exchange reaction mediated by Rad51

DNA strand exchange reaction is the central phase of homologous recombination. In eukaryote, Rad51, which is a RecA family recombinase binds single-stranded DNA and forms nucleoprotein filament named presynaptic filament. Presynaptic filament searches homologous sequence in double-stranded DNA (dsDNA) and promotes DNA strand exchange within homologous dsDNA. However, to accomplish the reaction, Rad51 requires some accessary proteins. Schizosaccharomyces pombe Swi5-Sfr1 complex is an accessary protein. Previous study demonstrated that Swi5-Sfr1 stimulates DNA strand exchange activity of Rad51 and stabilizes presynaptic filament active state in the presence of ATP. However, the role of Swi5-Sfr1 after presynaptic phase is unclear. To elucidate activation mechanisms of the reaction by Swi5-Sfr1 in detail, we established real-time observation system of DNA strand exchange. From our analysis, Swi5-Sfr1 stimulates DNA strand exchange reaction after presynaptic phase in the presence of ATP.

2A IWASAKI, Daichi ${ }^{1,2}$ HAYASHIHARA, Kayak ${ }^{1,2}$, -06 SHINOHARA, Miki ${ }^{1,2}\left({ }^{1}\right.$ Inst. Prot. Res., Osaka Univ., ${ }^{2}$ BioSci., Grad. Sch. Sci., Osaka Univ.)

The MRX complex ensures NHEJ fidelity through Xrs2-FHA? dependent Tel1 activation

Because DNA double-strand breaks (DSBs) is the most cytotoxic DNA lesion and often cause genomic instability, precise repair of DSBs is essential for maintenance of genomic stability. In yeast $\mathrm{Xrs} 2$, an ortholog of human Nbs1, is a multi-functional regulatory subunit of Mre11-Rad50-Xrs2 (MRX) complex, and its function is critical for DSB repair, whether by homologous recombination (HR) or non-homologous end joining (NHEJ). Previously, we reported that the Xrs2 FHA domain is required for NHEJ through the physical interaction with DNA ligase IV complex. Here we show that the Xrs2 FHA domain is required both to suppress of imprecise repair of DSBs, as well as to promote the robust activation of Tel1 in the DNA damage response pathway. The role of the FHA domain in Tel1 activation is independent of the Tel1-binding activity of the Xrs2 C-terminus, which mediates the recruitment of Tel1 to DSB ends. Both the Xrs2 FHA domain and Tel1 were required for the timely removal of the Ku complex from DSB ends to suppress imprecise repair. Thus, the Xrs2 FHA domain is involved not only in promotion of precise NHEJ, but also in suppression of imprecise repair to maintain DSB repair fidelity.
2B SATO, Tadashi ${ }^{2}$, HAMAGUCHI, Satoshi ${ }^{2}$, -01 SAKAIZUMI, Mitsuru ${ }^{2}$ KOBAYASHI, Tohru ${ }^{1}$, MYOSHO, Taijun ${ }^{1}$ ( ${ }^{1}$ The Dept. Sch. Food and Nutritional Sci., Shizuoka Univ., ${ }^{2}$ Inst. Sci. Tech., Niigata Univ.)

The sex-determining gene Dmy and a novel sex-determining gene $G s d f^{n e o Y}$ coexist in wild medaka population in Nagasaki

A conserved sex-determining gene Dmy have been identified in Japanese medaka. However, males without Dmy and females with Dmy were found in wild population. Recently, we found a sex-reversed XX male without Dmy in a wild population at Nagasaki, Japan. Sex-linkage analysis suggested that the responsible gene of XX male was Gsdf on linkage group 12. The null mutants with a frameshift mutation in Gsdf ${ }^{\text {neoY }}$ ( $G s d f$ on the neoY chromosome) coding region developed as sex-reversed females (XY females), indicating that $G s d f^{n e o Y}$ is the novel sexdetermining gene. In 2014, we also found 34 males with Dmy and 8 males $G s d f^{n e o Y}$ in a wild population of medaka at Nagasaki. Together, we revealed that two sex-determining genes, Dmy and $G s d f^{\text {neoY }}$, coexist in a wild medaka population at Nagasaki.

2B WATADA, Masayoshi ${ }^{1}$, YOSHIMURA, Kanamu ${ }^{1}$, -02 KAGAYAMA, Daisuke ${ }^{2}$ ( ${ }^{1}$ Grad. Sch. Sci. and Eng., Ehime Univ., ${ }^{2}$ Inst. AgroBiol. Sci., NARO)

Genetic analysis of the new sex ratio disorder found in Drosophila

One strain of Drosophila biauraria collected in Hokkaido several years ago showed sex ratio disorder, which produced only females every generation. This phenomenon is not reproduction by parthenogenesis, and females of the disorder strain need to be mated by males of another strains for production of females. Among 55 strains of $D$. biauraria collected in 2015 , we found that three strains of the species had the same characteristic, and this result showed that this phenomenon was polymorphic in natural environment in Hokkaido. We studied symbiotic infection by PCR method using standard primers of Wolbachia and Spiroplasma, and also checked the infection by antibiotic treatment. These studies cleared that the disorder was not caused by the infection of these symbionts. In addition, we injected body fluid of the disorder strain into the normal strain that produce females and males, and got strains that produced only females among the females of the normal strain, suggesting that this sex ratio disorder was caused by the cytoplasmic factor other than bacterial symbionts. In the present study, we showed that the factor seemed to be a RNA virus by the study of RNA-seq analysis. 
2B YOSHIDA, Kohta ${ }^{1}, \mathrm{KITANO}, \mathrm{Jun}^{1}\left({ }^{1}\right.$ Div. Ecological -03 Genet., Natl. Insitute Genet.)

A probabilistic model for karyotype evolution

Recent several reports suggest that karyotype evolution plays important roles in speciation and adaptive evolution. To investigate the functions and mechanisms of karyotype evolution, a probabilistic model applicable for phylogenetic comparative methods for karyotype evolution is needed. We established a probabilistic model based on centric fusions and fissions and pericentric inversions, which are major events of karyotype evolution. This model is useful for the phylogenetic comparative analyses of karyotype evolution of large taxonomic groups.
2B SEIKE, Taisuke ${ }^{1}$, NIKI, Hironori ${ }^{1}\left({ }^{1}\right.$ Microbial Genet. -05 Lab., Genet. Strains Res. Center, Natl. Inst. Genet.)

Mechanism of pheromone diversification in yeasts

Reproductive isolation is a key process of speciation. Recent studies have revealed that a few key mutations in pheromone/ receptor can give rise to a new species in the fission yeast Schizosaccharomyces pombe (Seike et al., Genetics, 2012; PNAS, 2015), but the mechanism of pheromone diversification in nature is unclear.

S. pombe has two mating types, Minus (M) and Plus (P). Mating is controlled by mating pheromones, M-factor and P-factor, secreted by M- and P-type cells, respectively. M-factor is encoded by three redundant genes, whereas P-factor is located at a single locus that encodes four copies of pheromone ORFs. Here, we examined 53 strains of $S$. pombe whose origins differ from that of the laboratory strain. Notably, all M-factor genes were highly conserved. In contrast, the P-factor gene was very diverse, resulting that a few amino acid substitutions and increased numbers of copies ( $>5$ copies) in the mature P-factor-coding region. Thus, recognition specificity of the M-factor is tight, whereas that of the P-factor is loose. Provably, the biased system for pheromone recognition might allow both tight recognition for partners and flexible adaptation in yeasts.

2B MUTO, Leona ${ }^{1}$, KAMIMURA, Yoshitaka ${ }^{2}$, TANAKA, -06 Kentaro ${ }^{1}$, TAKAHASHI, Aya ${ }^{1,3}{ }^{1}$ Dept. Biol. Sci., Tokyo Metropolitan Univ., ${ }^{2}$ Dept. Biol., Keio Univ., ${ }^{3}$ Res. Cent. Genomics and Bioinform, Tokyo Metropolitan Univ.)

Coevolution of genitalia between male and female in Drosophila suzukii : Effect of morphological diversification in ovipositor

Drosophila suzukii females have uniquely modified ovipositors, which have enabled them to lay eggs inside the hard skin of fresh fruits. Their ovipositors have more elongated but less curved shape compared to those of a closely related species, D. subpulchrella. In Drosophila spp., the ovipositor is also known to play an important role in copulation. During the copulation, female ovipositor is usually grasped by male genitalia. From a detailed observation of the genitalia of copulating pairs, it was confirmed that male claspers hold the posterior areas of female ovipositor in both species. Also, we found that a pair of male parameres with hook-shaped tips stab the female body during copulation in D. subpulchrella, but they do not reach the female body in D. suzukii. This suggests that D. suzukii may have lost the function of parameres due to elongation of the ovipositor in this species. Our observations suggest an intriguing possibility that coevolution in genitalia morphology between male and female in D. suzukii has been driven by changes in female ovipositor shape due to a shift in host fruit preference to fruits with much harder skins. 
2B SAWAMURA, Kyoichi ${ }^{1}$, WANG, Zhuo ${ }^{2}$, MIURA -07 Kohei ${ }^{2}$, HAYASHI, Takaaki ${ }^{2}$, HIRAI, Kazuyuki ${ }^{3}$, AWASAKI, Takeshi ${ }^{3}$, WADA, Moe ${ }^{4}$, KEIRA, Yoko ${ }^{4}$, ISHIKAWA, Hiroyuki O. ${ }^{4}{ }^{1}$ Faclt. Life and Env. Sci.s, Univ. Tsukuba, ${ }^{2}$ Grad. Sch. Life and Env. Sci., Univ. Tsukuba, ${ }^{3}$ Sch. Med., Kyorin Univ., ${ }^{4}$ Grad. Sch. Sci., Chiba Univ.)

Interaction among nucleoporins and reproductive isolation in Drosophila

In diverse organisms, intracellular localization of nuclear pore complex is determined by the interaction between Nup107-160 subcomplex (Y-complex Nups) and ELYS/MEL-28. Using CRISPR/Cas9, we disrupted the CG14215 gene, an X-linked Elys homolog of Drosophila melanogaster. Homozygous and hemizygous Elys mutants were viable but female sterile. Mutant females laid fertilized eggs but the eggs never developed. And Nup160, Nup96, and Nup37, but not other Y-complex Nups, were haplo-insufficient in the Elys mutation background. In other words, Elys/Y; Nup/+ and Elys/Elys; Nup/+ were lethal at the pupal stage. Furthermore, positive natural selection was detected in the Elys-like domain of the gene, especially in the branch for $D$. simulans, by the $\mathrm{Ka} / \mathrm{Ks}$ ratio test. Based on the phenotypic similarity to interspecific hybrid or introgression female sterility and inviability, we speculate the possible involvement of ElysNups interaction in reproductive isolation between $D$. melanogaster and D. simulans.

2B KUEI-YUEH, Ko ${ }^{1}$, HUANG, Hsuan-Cheng ${ }^{3}$, JUAN, -08 Hsueh-Fen ${ }^{2} \quad\left({ }^{1}\right.$ Genome \& Systems Biol. Degree Program, National Taiwan Univ., ${ }^{2}$ Dept. Life Sci., National Taiwan Univ., ${ }^{3}$ Inst. BioMed. Inform., National Yang-Ming Univ.)

Phylotranscriptomic patterns of network stochasticity and pathway dynamics during embryogenesis

Various evo-devo models have been proposed to describe the similarities and differences among developmental processes of different species. Although pieces of evidence indicate an evolutionary constraint during mid-developmental stage, its explanation and molecular mechanism are not fully understood yet. In our study, we aimed to tackle the problem via the tools of network and pathway analysis. Using the protein-protein interaction network information and gene expression data, we first measured the stochasticity within the biological network during the developmental process. Such analysis enables us to trace and compare the changes of network randomness among developmental processes of different species. In our result, a network reformation is observed during the development. The complexity of interconnections in the intermediate stages is higher than that in the early and late stages. Next, we sketched out the pathway dynamics during developmental process in order to narrow down our study from global network connections to detailed molecular regulations. With the systematic network view of developmental process, our results provided an alternative aspect of comparative embryology.
2B CHIH-KUAN, Chen ${ }^{1}$ ( ${ }^{1}$ Program in Inst. Ecol. -09 Evolutionary Biol., National Taiwan Univ.)

Regulatory Differences in Natal Down Development between Altricial Zebra Finch and Precocial Chicken

Birds are the most diversified terrestrial vertebrates, largely thanks to the diversification of feather. The hatchlings of altricial birds are almost naked, whereas those of precocial birds are covered with natal down. This difference is thought to reflect adaptation to different environments. However, the molecular basis is unclear. In zebra finch hatchlings, natal down partially covers the posterior dorsal (PD) skin, but is suppressed in the anterior dorsal (AD) skin. After analyzing the transcriptomes of $\mathrm{AD}$ and $\mathrm{PD}$ skins over three developmental stages, I found that the feather growth promoter $\mathrm{SHH}$ was expressed higher in PD skin than in AD skin. Moreover, the data suggested that the FGF/ MAPK signaling pathway is involved in natal down growth suppression and that FGF16 is an upstream signaling suppressor. Ectopic expression of FGF16 on chicken skin suppressed the feather bud elongation, similar to the phenotype found in zebra finch hatchlings. I proposed that FGF16-related signals cause the naked $\mathrm{AD}$ skin in zebra finch. My study provides insights into the regulatory divergence in natal down formation between precocial and altricial birds.

2B CHERIF, Amira ${ }^{3}$, MEDIOUNI BEN JEMÂA, Jouda ${ }^{3}$, -10 TAYLOR, Demar ${ }^{1}\left({ }^{1}\right.$ Lab. Appl. Entomol. and Zool., Univ. Tsukuba, ${ }^{2}$ Natl. Agronomic Inst. Tunisia (INAT), ${ }^{3}$ Lab. Biotechnol. Appl. to Agric., Natl. Agric. Res. Inst. of Tunisia (INRAT))

Molecular Characterization of Three Mayetiola Species (Diptera: Cecidomyiidae)

Cereal midges are considered as the most destructive pests of wheat, barley and oat worldwide. Species from the genus Mayetiola have been observed in the main cereal cultures of Tunisia. Some studies have studied $M$. destructor that attacks wheat and $M$. hordei that attacks barley. However, a third important species $M$. avenae, observed in oat, has never been studied and is not well documented in Tunisia.

This study aimed to distinguish between the 3 species of midges by molecular characterization of a region of the cytochrome oxidase subunit I (COI) gene commonly used to reveal genetic diversity between gall midges. A set of 30 individuals, 10 from each species, was used and three primer sets tested. Only one primer pair allowed us to distinguish between the different species. The phylogenetic tree clearly showed significant separation of the three different species into dissimilar clades. Each clade contained only specimens from the same species. Our results indicate this area of the COI gene can be used as a marker to reveal genetic diversity between species of Mayetiola. 
2C ZHOU, Hua ${ }^{1}$, HIRATA, Megumi ${ }^{1}$, OSAWA, Ryo ${ }^{1}$, -01 FUJINO, Kaien ${ }^{1}$, KISHIMA, Yuji ${ }^{1}\left({ }^{1}\right.$ Hokkaido Univ.; Res. Faclt. Agr.; Lab. Plant Breeding)

Epigenetic-independent regulation of Tam3 in Antirrhinum majus detains transposase to the plasma membrane by targeting its BED-zinc finger domain

Transposable elements (TEs) are considered to be parasites of host genomes as they act as powerful mutagens. If not kept in check, they can cause gene disruption, genome rearrangement, and genomic takeover. Hence, activities of TEs are under rigid control of hosts. Up to now, all discovered TE regulations have been epigenetic-dependent, with the exception of the DNA transposon Tam3. Blocking nuclear translocation of Tam3 transposase (TPase) is consistent with the suppression of Tam3 in Antirrhinum majus. Our data uncovered that epigenetic-independent regulation of Tam3 is mediated by the BED-zinc finger domain of Tam3 TPase. The host targets the N-terminus of the BED-zinc finger domain, which contains two highly conserved aromatic amino acids, to detain Tam3 TPase to the plasma membrane, and to silence Tam3. Zinc finger proteins perform broader functions in transcriptional regulation through their DNA binding ability. Here, our results revealed epigeneticindependent silencing against TE activity in a post-translational manner was due to the protein binding ability of BED-zinc finger domain.
2C TSUGANE, Kazuo ${ }^{1,2}$, TSUGANE, Mika ${ }^{3}$, MAEKAWA, -03 Masahiko ${ }^{4}{ }^{1}$ Natl. Inst. Basic Bio, ${ }^{2}$ SOKENDAI, ${ }^{3}$ Nagoya Univ., ${ }^{4}$ Okayama Univ.)

The gain-of-function mutations in rice caused by insertion of the DNA transposon, nDart1

DNA transposons used cut and paste manner for transposition, occupied $13 \%$ of rice genome. The endogenous non-autonomous transposon, $\mathrm{nDart1elements} \mathrm{tend} \mathrm{to} \mathrm{insert} \mathrm{into} \mathrm{genic} \mathrm{regions}$ under natural growth conditions. The dominant mutants were occasionally isolated from the nDart1-promoted tagging line. Efficient selection and analysis of dominant mutants to analyze the gene functions in rice is very useful. A semidominant mutant, Bushy dwarf tiller1 (Bdt1), which has the valuable agronomic traits of multiple tillering and dwarfism, was obtained from the tagging line. The Bdt1 mutant carried a newly inserted nDart1 at 38-bp upstream of the transcription initiation site of a nonprotein-coding gene, miR156d. This insertion caused an upstream shift of the miR156d transcription initiation site and, consequently, increased the functional transcripts producing mature microRNAs. These results indicate that the total amount of miR156d is controlled not only by transcript quantity but also by transcript quality. This study demonstrates the ability of nDart1 to produce gain-of-function mutants as well as further insights into the function of transposable elements in genome evolution.

\section{C HAYASHI, Yui ${ }^{1}$, MASUDA, Yukari ${ }^{2}$, KATO, Atsushi ${ }^{2}$, -04 ITO, Hidetaka ${ }^{2}\left({ }^{1}\right.$ Grad. Sch. Life Sci., Hokkaido Univ., ${ }^{2}$ Faclt. Sci., Hokkaido Univ.)}

A transpositional regulation of a heat-activated retrotransposon in Arabidopsis

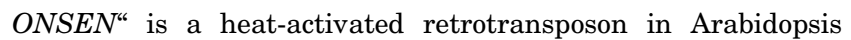
thaliana. Previously we showed that transcript level of ONSEN was increased by heat stress and transposition of ONSEN was observed in the progeny of stressed plants that deficient in siRNA biosynthesis. In this study 
2C NOZAWA, Kosuke ${ }^{1}$, MASUDA, Seiji ${ }^{1}$, KATO, -05 Atsushi ${ }^{2}$, ITO, Hidetaka ${ }^{2}{ }^{1}$ Grad. Sch. Life Sci. Hokkaido Univ., ${ }^{2}$ Faclt. Sci., Hokkaido Univ.)

Analysis of transcriptional regulation of heat-activated retrotransposon

Transposable elements are major components of plant genomes and most of them are silenced by epigenetic modifications. However, recent studies showed the environmental stress induces some transpositions. ONSEN is an Ty1/copia-type retrotransposon in Arabidopsis. ONSEN became transcriptionally active and synthesized extrachromosomal DNA subjected to heat stress. Moreover, high frequency of new insertions was observed in the progenies of stressed mutants that deficiency of siRNA biosynthesis. In Arabidopsis, ONSEN is conserved among accessions. We found that a transcript level of the heat-activation of ONSEN was various among accessions. The copy number and a cis element of the heat-activation of ONSEN were analyzed among accessions. We report the result of a comparative analysis of the transcriptional regulation of ONSEN in the accession.
2C INOUE, $\mathrm{Kota}^{2}$, ICHIYANAGI, Kenji ${ }^{1}, \mathrm{FUKUDA}, \mathrm{Ke}^{3}$, -07 GLINKA, Michael ${ }^{4}$, SASAKI, Hiroyuki ${ }^{2}$ ( ${ }^{1}$ Grad. Sch. Bioagricultural Sci., Nagoya Univ., ${ }^{2}$ Med. Inst. Bioregulation, Kyushu Univ., ${ }^{3}$ Cell memory lab., RIKEN, ${ }^{4}$ Faclt. Sci., Univ. of Bristol)

Switching from PTGS to TGS for epigenetic regulation of retrotransposons during male germ cell development in mice

During male germ cell development in mice, both DNA methylation and PIWI-interacting small RNAs (piRNAs) are involved in regulation of retrotransposon activity. The piRNA system destroys retrotransposon-derived RNAs and guides de novo DNA methylation at some retrotransposon promoters in prospermatogonia. However, it remains unclear whether DNA methylation contributes to retrotransposon silencing in this cell type. We performed DNA methylome and transcriptome analysis on developing male germ cells from Pld6 and Dnmt3l knockout mice, which are defective in piRNA biogenesis and de novo DNA methylation, respectively. The results revealed that, in spermatogonia, DNA methylation plays a very minor role in retrotransposon regulation, while the piRNA system plays an important role by a post-transcriptional mechanism. However, DNA methylation had a long-term effect: hypomethylation caused by the Dnmt3l mutation resulted in retrotransposon activation in later stages of development (spermatocytes), and transcriptional silencing by DNA methylation became more important in spermatocytes, indicating a developmentally programed switch of mechanisms to combat retrotransposons.

2C NAKAGAWA, So ${ }^{1,2}$, TAKAHASHI, Mahoko Ueda ${ }^{2}$ -08 ( ${ }^{1}$ Dept. Molec. Life Sci., Tokai Univ. Sch. Med., ${ }^{2}$ Micro/ Nano Technol. Center, Tokai Univ.)

gEVE, genome-scale endogenous viral element database, and its applications

In mammals, approximately $10 \%$ of genome sequences correspond to endogenous viral elements (EVEs) including endogenous retroviruses (ERVs), which are thought to be derived from viral infections in germ cells. Although most EVEs have been inactivated by insertions, deletions, substitutions, and/or epigenetic modifications, a few open reading frames (ORFs) of EVEs are still active and express viral proteins in the hosts. Indeed, several genes derived from EVEs have been found to be functional for host species, such as syncytins for placenta development in various mammals. However, no databases of EVE ORFs are available, and therefore molecular functions and evolutionary pathways of EVEs have not yet been understood comprehensively. Hereby, I developed EVE ORF databases for 19 mammalian species (http://geve.med.u-tokai.ac.jp) containing a total of 736,771 non-overlapping EVE ORFs. The gEVE database provides nucleotide and amino acid sequences, genomic loci and functional annotations of EVE ORFs. In this talk, I will introduce the gEVE database as well as its application to the various transcriptome analyses with various large-scale sequencing data. 
2C NISHIHARA, Hidenori ${ }^{1}\left({ }^{1}\right.$ Dept. Life Sci. and Technol., -09 Tokyo Inst. Tech.)

Evolution of cis-regulatory network involving transposable elements in mammals

It is largely unknown how much transposable elements (TEs) have involved in the morphological evolution of mammals. In the present work, I analyzed the ChIP-seq data of human to reveal the binding sites of ER-alpha and several transcription factors that are responsible for the development of mammary gland in mammals, and compared those overlapping with TEs. It is revealed that the binding sites of these factors are significantly biased within several kinds of TEs which contain the binding motif in the consensus sequences. Genomic regions where the TEderived putative functional elements locate in the higher density are the promoter regions of several genes, most of which are known to be involved in the development of mammary gland. In addition, two waves of retrotransposition bursts had produced the major TE-derived functional elements during mammalian evolution. Therefore, these TE families had propagated the sources of the transcription factor binding sites via retrotransposition and have contributed to increase the cis-regulatory elements involved in mammary gland evolution.
2D YOSHIMI, Kazuto ${ }^{1}$, IMAI, Yuji ${ }^{1}$, TANAVE, Akira ${ }^{1}$, -01 MASHIMO, Tomoji ${ }^{2}$, KOIDE, Tsuyoshi ${ }^{1}$ ( ${ }^{1}$ Mouse Genomics Resource Lab., Natl. Inst. Genet., ${ }^{2}$ Inst. Exp. Anim. Sci., Grad. Sch. Med., Osaka Univ.)

Long single-stranded oligonucleotide-mediated knock-in with CRISPR/Cas9 in rodents

Genome editing technologies have enabled generating genetically modified animals within the past few years. Development of an efficient knock-in (KI) technology will facilitate easy and flexible genome engineering to introduce precise mutations or genetic modifications at any target sites in many species.

We have reported the efficient generation of several types of KI rats using single-stranded oligonucleotides (ssODNs), such as SNP substitution, small DNA fragment insertion, and DNA fragment elimination of a $7 \mathrm{~kb}$ retrotransposon element (Yoshimi $\mathrm{K}$ et al., Nat Commun 2014). It is easy to design and synthesize ssODNs as a DNA donor, while the limitation of ssODN-mediated $\mathrm{KI}$ is the maximum length of the insert DNA, which is less than almost $200 \mathrm{bp}$.

In this study, we developed a new strategy to generate long ssODN fragments (lsODNs) using nicking endonucleases. Microinjection of a synthesized lsODN with gRNA and Cas9 mRNA produced several types of KI rodents, such as GFP-KI rats at Thy1 locus and floxed-KI mice at Tyr locus. LsODN-mediated KI technologies can apply to any target site of any species, thus simplifying genome engineering in living organisms.

2D TESHIGAWARA, Rika ${ }^{1,2}$, HIRANO, Kunio ${ }^{1}$, -02 NAGATA, Shogo ${ }^{1}$, AINSCOUGH, Justin ${ }^{3}$, TADA, Takashi ${ }^{1}$ ( ${ }^{1}$ Dept. Stem Cell Eng., Field of Stem Cell Res. Inst. Front. Med. Sci., Kyoto Univ., ${ }^{2}$ Grad. Sch. Med., Kyoto Univ., ${ }^{3}$ Dept. Biol., Univ. of York)

OCT4 activity during conversion of human intermediately reprogrammed stem cells to iPSCs through mesenchymalepithelial transition

To facilitate understanding the mechanisms of somatic reprogramming to human induced pluripotent stem cells (iPSCs), we have established intermediately reprogrammed stem cells (iRSCs), human mesenchymal cells that express exogenous Oct4, Sox2, Klf4 and c-Myc (OSKM) and endogenous SOX2 and NANOG. iRSCs can be stably maintained at low density. At high density, however, they are induced to enter mesenchymalepithelial transition (MET), resulting in reprogramming to an iPSC state. Morphological changes through MET correlate with silencing of exogenous OSKM, and upregulation of endogenous OCT4. A CRISPR/Cas9-mediated GFP knock-in visualized the temporal regulation of endogenous OCT4 in cells converting from iRSC to iPSC state. OCT4 activation coincident with silencing of OSKM occurred prior to entering MET. Notably, OCT4 instability was frequently observed in cells of developing post-MET colonies until a late stage (>200 cells), demonstrating that OCT4activated post-MET cells switched from asymmetric to symmetric cell division in late stage reprogramming. 
2D MAKINO, Shigeru ${ }^{1}$, GONDO, Yoichi $^{1} \quad\left({ }^{1}\right.$ RIKEN -03 BioRes. Cent.)

Mechanisms of translation reinitiation by frameshifted mutant alleles

Post-termination ribosomes occasionally reinitiate translation from an AUG codon downstream of the termination codon. It has been, however, unknown what extent translation reinitiation generally occurs. We generated mutant alleles carrying out-offrame indel mutations by CRISPR-Cas9 in the mouse NIH3T3 cell line. Unexpectedly, we found that the translation was reinitiated from an AUG codon other than the native translation start codon in all these frameshifted alleles that we established. In this study, we found that translation reinitiation is not limited to the mouse cells but may occur universally irrespective of the species difference in the presence of a premature termination codon.

Translation reinitiation also occurs in wild-type genes as uORFs, which are found in almost half of human transcripts. However, the functions of uORFs have not been understood except several cases. Detailed analysis of the frameshifted cell lines reveals a critical regulatory role of translation reinitiation in gene expression as a new paradigm in the central dogma.
2D KISHI, Nobuyuki ${ }^{1}, \mathrm{HARA}, \mathrm{Satoshi}^{2}$, TAKADA, Shuji ${ }^{2}$, -05 GOTO, Yuji ${ }^{1}$, KUBOTA, Souichirou ${ }^{1}$ ( ${ }^{1}$ Lab. Molec. Genet. and CytoGenet., Dept. Biol., Faclt. Sci., Toho Univ., ${ }^{2}$ Dept. Systems BioMed., Natl. Res. Inst. for Child Health and Dev.)

Small RNA profiling and detection of piRNA from testicular germ cells in the inshore hagfish

It is known that chromosome elimination occurs during early embryogenesis in eight hagfishes. Previous studies indicated the chromosomes restricted to germ cells in these hagfishes are generally heterochromatic and mosaics of various highly repetitive DNA. Recently, Piwi-interacting RNAs (piRNAs), a class of small RNA of 24-30 nucleotides long that are predominantly transcribed in germ cells of broad species from arthropods to mammals, were involved in heterochromatinization with heterochromatin protein 1 in Drosophila. Thus we profiled the small RNAs expressed in testis and liver of inshore hagfish, Eptatretus burgeri using next generation sequencer. We obtained 91,000 unique sequences of 26-30 nucleotide small RNAs. Most of those showed the several features of piRNAs, although they had no obvious homology with known small RNAs. By semi-quantitative RT-PCR analysis using testis and liver, 16 of top 20 piRNA candidates were exclusively transcribed in testis. Some of these candidates showed partial homology to germline-restricted highly repetitive sequences, implying piRNA-mediated heterochromatinization of the chromatin harboring those repetitive sequences in the hagfishes.

2D KITANO, Shohei ${ }^{1,2}$, AIZAWA, Yasunori ${ }^{3,4}\left({ }^{1}\right.$ Dept. Life -06 Sci., ${ }^{2}$ Educ. Academy of Computational Life Sci., Tokyo Inst. Technol., ${ }^{3}$ Grad. Sch. Life Sci. and Technol., Tokyo Inst. Tech., ${ }^{4}$ Cent. Biol. Resources and Inform.)

Functional analysis on a highly conserved and upstream ORF-encoded protein in mice

Mammalian mRNAs are believed to be monocistronic for decades. Recent proteomic studies however revealed that thousands of open reading frames located on the 5' untranslated regions (upstream ORFs, uORFs) are translated in vivo. However, no uORF that encodes bioactive protein has been discovered yet. In this study, we discovered an uORF-derived small protein that is highly conserved among vertebrates and specifically expresses in brain. In this presentation, we will discuss functionality of this uORF-derived protein by showing the latest data on our mouse model. 
2D NAGAO, Michiaki ${ }^{1}$, TSUCHIYA, Fumina ${ }^{2}$, -07 MOTOHASHI, Reiko ${ }^{3}$, ABO, Tatsuhiko ${ }^{4,5}\left({ }^{1} \mathrm{Grad}\right.$. Sch. Sci. and Tech., Okayama Univ., ${ }^{2}$ Dept. Biol. Env. Sci., Faclt. Agr., Shizuoka Univ., ${ }^{3}$ Lab. Molecular Breeding, Coll. of Agric., Shizuoka Univ., ${ }^{4}$ Grad. Sch. Sci. and Tech., Okayama Univ., ${ }^{5}$ Dept. Biol., Faclt. Sci., Okayama Univ.)

Release factor homologs of Arabidopsis thaliana

Non-stop mRNAs, which lack in-frame stop codon, frequently occur in cells. When it is translated, ribosome stalls at the end of it to form non-productive translation complex (NTC). Accumulation of NTCs is supposed to be deleterious, and bacteria are known to have at least one of three ribosome rescue factors, tmRNA, ArfA or ArfB, to dissolve them. Among these, ArfB is a homolog of class I release factors. Chloroplasts and mitochondria are thought to originate in bacteria, and have bacteria-like translation system. While no functional tmRNA or ArfA homologs have been so far reported in both organelles, ICT1, human mitochondrial ArfB homolog, is essential for human cells, suggesting that ArfB homolog is the primary ribosome rescue factor in organelle. Here, we surveyed ArfB homologs from Arabidopsis thaliana genome. One of the RF homologs surveyed is highly homologous to ArfB and ICT1 and has their characteristic features such as GGQ motif and unstructured C terminal region rich in basic amino acids. Moreover, it showed ribosome rescue activity in Escherichia coli cell. These suggest that it functions as the ribosome rescue factor in plant organelles.

2D KUTSUNA, Shinsuke ${ }^{1}$, SEGAWA, Yuki ${ }^{1}$, KOIKE -08 Anna ${ }^{1}$, MUROYA, Mitsuhiko ${ }^{1}$, ONAI, Kiyoshi ${ }^{2}$, ISHIURA, Masahiro ${ }^{2}$, MANABE, Katsushi ${ }^{1}\left({ }^{1}\right.$ Bionano System Sci., Yokohama City Univ., ${ }^{2}$ Gene Res., Nagoya Univ.)

Analysis of flower movement mutants and seedling clock mutants

Previous reports indicates that the circadian clock regulates the diurnal petal movement in several plant species, such as morning glory and kalanchoe. However, the relationship between the clock and the diurnal movement is remained to be determined at Molec. Genet. level. In Arabidopsis thaliana, we had isolated several flower movement mutants named as Y-shaped curvature at flower closure. These show low amplitude flower opening and closure. Here, we will report that the flower movement mutants ycu1, 2, and 3 exhibit several circadian phenotypes under continuous light condition. The first, their seedlings shows loss of sustainability of the petiol circadian rhythm. In addition, gene expression profiles in flower organ in the mutants lose circadian rhythmisity. These results suggest that deep linkage between the molecular circadian gene expression and diurnal movements in the plant. In this meeting, we would like to discuss about the clock gene expressions and the movements.
2D NISHIZAKI, Eri ${ }^{1}$, MORITA, Akitsugu ${ }^{1}$, YAMAGUTI, -09 Akimitsu ${ }^{1}$, KITAYAMA, Yoko ${ }^{2}$, OYAMA, Tokitaka ${ }^{3}$, KONDO, Takao ${ }^{2}$, KUTSUNA, Shinsuke ${ }^{1}\left({ }^{1} \mathrm{Grad}\right.$. Sch. NanoBiosci., Yokohama City Univ., ${ }^{2}$ Div. Biological Sci., Grad. Sch. Sci., Nagoya Univ., ${ }^{3}$ Div. Biol. Sci., Grad. Sch. Sci., Kyoto Univ.)

Spatiotemporal analysis of circadian clock protein homolog in the cyanobacterium Prochlorococcus marinus str. NATL1A

Many organisms on the earth has circadian clock of about 24-h period. They control their biological activities by circadian clock and fit a day round environmental change. Cyanobacteria are photosynthetic prokaryotes known to have a circadian clock. Synechococcus elongatus PCC 7942 is the kind of cyanobacteria for which most bacterial circadian research has been conducted. Many studies of them show that thier circadian clock consists of three clock protein, KaiA, KaiB, and KaiC. Marine cyanobacteria, Prochlorococcus marinus str. NATL1A (hereafter P. marinus), used in this research. They are the most abundant photosynthetic organisms on Earth. They lost KaiA in the process of the evolution. Homologs of $k a i B$ and kaiC is maintained in the genome of many kinds of Prochlorococcus, but it's still unclear whether P. marinus has the circadian rhythm. In this study, we analyze the spatiotemporal expression of $P$. marinus $\mathrm{KaiB}$ and KaiC proteins by Western blot. As a result, it is suggested that both KaiB and KaiC expression place in the cell moves in a day.

2D KOBAYASHI, Yuto ${ }^{1}$, RYO, Masashi ${ }^{1}$, OSHIKOSHI, -10 Yuta $^{1}$, IDEGUCHI, Yamato ${ }^{1}$, KUWANO, Takashi ${ }^{1}$, TEZUKA, Takafumi ${ }^{1}$, AOKI, Setsuyuki ${ }^{1}$ ( ${ }^{1}$ The Complex Systems Dept., Grad. Sch. Inform. Sci., Nagoya Univ., ${ }^{2}$ Sch. Inform. and Sci.s, Nagoya Univ.)

Bioluminescence bursts in Escherichia coli luciferase reporter strains

We introduced the firefly luciferase gene as a transcriptional reporter into an Escherichia coli WT strain. All the resulting reporter strains exhibited an abrupt and large increase of bioluminescence immediately before the onset of the stationary growth phase. This "bioluminescence burst" was not promoterspecific and did not correlate with changes in the levels of luciferase mRNAs or luciferase proteins. Therefore, the bioluminescence burst is generated by reflecting changes in a posttranslational process(es). We are currently studying the changes in some metabolites and bioluminescence profiles of some geneknockout reporter strains, which lack genes potentially involved in the bioluminescence reaction by the luciferase protein. 
2E SONODA, Satoru ${ }^{1}$, OHTA, Akane ${ }^{1}$, MARUO, Ayana ${ }^{1}$, -01 UJISAWA, Tomoyo ${ }^{1}$, KUHARA, Atsushi ${ }^{1}\left({ }^{1}\right.$ Lab. Mol. and Cell. Regul., Faclt. Sci. Eng., \& Inst. Integr Neurobiol, Konan Univ.)

Feedback system between sperm and temperature sensingneuron in cold acclimation of $C$. elgans

Temperature acclimation in animals is important mechanism for survival and proliferation. To investigate temperature acclimation, we are using cold acclimation in Caenorhabditis elegans. $20^{\circ}$ C-cultivated animals did not survival at $2^{\circ} \mathrm{C}$. In contrast, $15^{\circ} \mathrm{C}$ cultivated animals can survive. Previously reported, this cold acclimation is regulated by ASJ temperature sensing-neuron, which releases insulin that is received by intestine and neuron (Ohta, Ujisawa et al., Nature commun, 2014). To identify genes in downstream of insulin-signaling, we performed DNA microarray analysis. We found that expressions of sperm genes were changed in insulin receptor mutants, and sperm mutants showed abnormal cold acclimation. Unexpectedly, genetic epistasis analysis suggested that abnormal cold acclimation of sperm mutant was suppressed by the mutations in ASJ temperature sensing-neuron. Calcium imaging analysis showed that ASJ neuronal activity in response to temperature was decreased in sperm mutant gsp-4, and rescued by expressing gsp-4 gene in sperm. Thus, we propose a novel feedback between sperm and ASJ temperature sensing-neuron in cold acclimation of $C$. elgans.

2E OKAHATA, Misaki ${ }^{1}$, OHTA, Akane ${ }^{2}$, MINAKUCHI, -02 Yohei $^{3}$, TOYODA, Atsushi ${ }^{3}$, KUHARA, Atsushi ${ }^{2}$ ( ${ }^{1}$ Dept. Biol., Grad. Sch. Natural Sci., Konan Univ., ${ }^{2}$ Inst. Integr Neurobiol, Konan Univ., ${ }^{3}$ Natl. Inst. Genet.)

Genetic analysis of natural variation and potassium channel in cold acclimation

Temperature is important environmental factor for animals. We are studying cold acclimation of natural variants in C. elegans. $\mathrm{AB} 1$ from Australia can rapidly acclimate to new temperature, whereas CB4856 from Hawaii can slowly acclimate. To identify the responsible gene polymorphism, we decoded genome sequence of three natural variants including $\mathrm{AB} 1$. We made many recombinants by crossing $\mathrm{AB} 1$ with $\mathrm{CB} 4856$. We performed SNP and phenotype analysis of these RI lines. Responsible polymorphism was mapped between 1.1 and $5.1 \mathrm{cM}$. We are going on another study to identify new genes involved in cold acclimation, by using the information of previous DNA microarray analysis. So far, mutant defective in $k q t$-2 encoding potassium channel showed abnormal cold acclimation. The expression of KQT-2 was observed in a pair of head sensory neurons and intestine.
2E IOROI, Makoto ${ }^{1}$, FUJII, Satoko ${ }^{2}$, INOUE, Tomoka ${ }^{2}$, -03 KUHARA, Atsushi ${ }^{2}$, OHTA, Akane ${ }^{2}$ ( ${ }^{1}$ Dept. Biol., Grad. Sch. Natural Sci., Konan Univ., ${ }^{2}$ Inst. Integr. Neurobiol., Konan Univ.)

CRH-1/CREB and NCS-1 regulate cold acclimation speed in C. elegans

Temperature acclimation is important for life and proliferation of animals. C. elegans changes their cold tolerant status depending on previous ambient temperature. We found that the cold tolerant status is overwritten in only three hours through CRH1/CREB in neurons. We call this phenomenon as a cold acclimation. Our calcium imaging implies that ASJ temperature sensoryneuron itself alters temperature response depending on cultivation temperature, which is modulated by CREB. In order to identify the site of action of CREB in cold acclimation, we expressed CREB cDNA in a various sets of neurons in CREB mutant. As a result, abnormal delay of cold acclimation in CREB mutant was rescued by the expression of CREB cDNA in both ASJ sensoryneuron and RMG interneuron. Recently, we found that ncs-1 mutant defective in Neuronal Calcium Sensor (NCS) showed abnormal delay of cold acclimation. NCS-1 is expressed in thirteen head neurons including RMG interneuron. Genetic epistasis analysis indicates that CRH-1/CREB and NCS-1 accelerate cold acclimation in the different pathways. We are now trying to identify whether function of NCS-1 in RMG is necessary for cold acclimation.

2E TAKAGAKI, Natsune ${ }^{1}$, OHTA, Akane $^{2}$, MINAKUCHI, -04 Yohei $^{3}$, TOYODA, Atsushi ${ }^{3}$, KUHARA, Atsushi ${ }^{2}$ ( ${ }^{1}$ Dept. Biol., Grad. Sch. Natural Sci., Konan Univ., ${ }^{2}$ Inst. Integr. Neurobiol, Konan Univ., ${ }^{3}$ Natl. Inst. Genet.)

Xanthine metabolism regulates cold acclimation of C. elegans

We are aiming at the elucidation of temperature acclimation mechanism. Wild-type animals can survive at 2 degree after cultivation at 15 degree, however, 25 degree-cultivated animals can not survive at 2 degree. We found that a novel mutation, chr1, causes abnormal cold acclimation. To identify responsible gene of this mutation, we performed SNP analysis by using next generation sequencer. As a result, F55B11.1 gene encoding Xanthine dehydrogenase (XDH) was the responsible gene for defective cold acclimation, and F55B11.1(ok3234) null mutant showed defect in cold acclimation. Defective cold acclimation of F55B11.1(ok3234) mutants were rescued by F55B11.1 genomic gene. Furthermore, F55B11.1::VENUS reporter gene was expressed in socket glial cells in the head (OLQso), excretory cell and intestine. We are now introducing a series of cell-specific rescue experiments. Since Xanthine dehydrogenase (XDH) is known to produce uric acid that is an antioxidant of xanthine, F55B11.1 mutant probably reduces capacity to detoxify ROS. We therefore hypothesizes that cell membranes are damaged and cell death increases, so F55B11.1 mutant might become sensitive to cold stimulation. 
2E ARAI, Mary ${ }^{1}$, INOUE, Akitoshi ${ }^{1}$, ISHIHARA, -05 Takeshi ${ }^{1}\left({ }^{1}\right.$ Dept. Biol., Faclt. Sci., Kyushu Univ.)

Analyses of the regulation of forgetting by the food signals in the olfactory learning of C. elegans

In C. elegans, TIR-1/JNK-1 pathway in AWC sensory neurons accelerates the forgetting of the adaptation to diacetyl, which is sensed by AWA sensory neurons. In wild-type animals, the adaptation is sustained for less than 4 hours on food after adaptation, whereas, in tir-1 loss-of-function mutant, the adaptation persists more than a day in the same condition. However, when, after conditioned with diacetyl, animals are incubated without food, both wild-type and tir-1 (lf) animals are recovered from the adaptation within 4 hours. Therefore, we postulated that food signals, which are inhibited by TIR-1/JNK-1 pathway in AWC, inhibit forgetting. To elucidate the mechanisms for the regulation of the forgetting by the food signals, we carried out two different screenings from tir-1 $(l f)$. In one screening, we searched for the mutants showing the normal forgetting even when animals were cultivated on food after conditioning. In another screening, we searched for the mutants showing the prolonged adaptation even when animals were incubated without food. From these two screenings, we isolated 10 mutant lines as candidates.
2E MURAKAMI, Hinata ${ }^{1}$, TANAKA, Ryoya ${ }^{1}$, HIGUCHI, -07 Tomohiro ${ }^{1}$, ITO, Hiroki ${ }^{1}$, SATO, Kosei ${ }^{1}$, YAMAMOTO, Daisuke $^{1} \quad{ }^{1}$ Div. NeuroGenet., Dept. Dev. Biol. NeuroSci. Grad. Sch. Life Sci., Tohoku Univ.)

Attempts at producing genetic tools for the analysis of Drosophila subobscura-specfic courtship behaviors

In two species belonging to the genus Drosophila, Drosophila subobscura exhibits a particularly exotic behavior; a male gives his digests to a potential mate as a "nuptial gift", a behavior not observed in D. melanogaster. For the understanding of the neural mechanism underlying such a unique component in courtship behavior, we attempt to generate transgenic $D$. subobscura flies, in which neuronal cluster-specific expression of the thermosensitive dTrpA1 channels can be induced with GAL4/UAS and FLP/ FRT systems. In D. melanogaster, the fruitless (fru) gene is considered as the master regulator of the male courtship circuitry formation. On the assumption that fru plays a similar role in $D$. subobscura, we are generating several transgenic flies in $D$. subobscura that include: fru-GAL4, UAS-dTrpA1, UAS>stop $>d T r p A 1$ and $h s-F L P$. We have succeeded in generating the fruitless-GAL4 line by means of the CRISPR/Cas9 system. In addition, we already have multiple attP lines in D. subobscura, so that $U A S-d T r p A 1, U A S>$ stop $>d \operatorname{TrpA1}$ and $h s-F L P$ can be integrated individually into an $a t t P$ docking site.

2E UEYAMA, Morio ${ }^{1,2}$, ISHIGURO, Taro ${ }^{2,3,4}$, GENDRON, -08 Tania F. ${ }^{4}$, FUJIKAKE, Nobuhiro ${ }^{2}$, KONNO, Takuya ${ }^{5}$, KOYAMA, Akihide ${ }^{5}$, ONODERA, Osamu ${ }^{5}$, ISHIKAWA, Kinya ${ }^{3}$, WADA, Keiji ${ }^{2}$, PETRUCELLI, Leonard ${ }^{4}, \quad$ NAGAI, Yoshitaka ${ }^{1,2} \quad\left({ }^{1}\right.$ Dept. Neurotherapeutics, Osaka Univ. Grad. Sch. Med., ${ }^{2}$ Dept. Degenerative Neurological Diseases, Natl. Inst. NeuroSci., Natl. Cent. Neurol and Psychiatry, ${ }^{3}$ Dept. Neurol and Neurol. Sci., Grad. Sch. Med. and Dental Sci, Tokyo Med. and Dental Univ., ${ }^{4}$ Dept. NeuroSci., Mayo Clinic, ${ }^{5}$ Dept. Neurol., Clinical NeuroSci. Branch, Brain Res. Inst., Niigata Univ.)

Toxicity of dipeptide repeat proteins in C9 ALS/FTD model fly

An abnormal expansion of $\mathrm{G} 4 \mathrm{C} 2$ repeat within the C9ORF72 gene has been found to be the most common genetic mutation for amyotrophic lateral sclerosis (ALS)/ frontotemporal dementia (FTD). In the brain of C9 ALS/FTD patients, the expanded repeat RNA transcribed from mutated gene leads to the formation of RNA aggregates, namely RNA foci, and is also translated into dipeptide repeat (DPR) proteins by the repeat associated nonATG (RAN) translation. Although these RNA foci and/or DPR proteins are involved in the neurodegeneration, the molecular mechanisms leading to ALS/FTD pathogenesis remain unclear. To dissect the pathogenic roles of expanded repeat RNA and DPR proteins, we established novel Drosophila models of ALS/FTD expressing both expanded G4C2 repeat RNA and DPR proteins, or expressing only expanded $\mathrm{G} 4 \mathrm{C} 2$ repeat RNA. We found that flies expressing both expanded repeat RNA and DPR proteins in the eye or in neurons showed severe rough eye phenotype or motor dysfunction, respectively, while flies expressing only expanded repeat RNA exhibited normal eye or normal motor function, respectively. These results indicate that DPR proteins mainly contribute to the neurodegeneration. 
2E LEON, Julio ${ }^{1}$, MORIOKA, Noriko ${ }^{1}$, SHENG, Zijing ${ }^{1}$, -09 NAKABEPPU, Yusaku ${ }^{1}$ ( ${ }^{1}$ Div. Neurofunctional Genomics, Med. Inst. Bioregulation, Kyushu Univ.)

Mechanisms of Neurodegeneration induced by a DNA repair enzyme, MUTYH

We have shown that 8-oxoguanine accumulation in nuclear or mitochondrial DNA in brains under oxidative conditions is highly associated with neurodegeneration, and that MTH1 (8-oxodGTPase) and OGG1 (8-oxoguanine DNA glycosylase) protect brains by preventing the 8-oxoguanine accumulation, while MUTYH (adenine DNA glycosylase) accelerates neurodegeneration. Isolated neurons from wild-type and MTH1/OGG1-deficient mice exhibited efficient neuritogenesis in the presence of antioxidants, however, in the absence of antioxidants MTH1/ OGG1-deficient neurons increased 8-oxoguanine accumulation in their mitochondrial DNA, and exhibited significantly poor neuritogenesis, indicating that MUTYH induces neuronal dysfunction when 8-oxogianine accumulated in mitochondrial DNA in neurons. Oxidative stress markedly activated microglia isolated from wild-type but not MUTYH-deficient brains, and only the former significantly induced neuronal death in coculture experiments. These results indicate that MUTYH induces neuronal dysfunction and microglial activation, independently, and thus accelerates neurodegeneration.

2E OKADA, Norihiro ${ }^{1}$ ( ${ }^{1}$ Found. Adv. InterNatl. Sci., $-10 \quad{ }^{2}$ National Cheng Kung Univ.)

What is fighting? Analysis of its neurogenomic state

We are going to elucidate what the fighting is from a viewpoint of molecular biology by using so-called fighting fish (Betta splendens). In this case, we mean the fighting between the same species, but not between different species. The fighting between the same species is territorial behavior and is believed to be conserved during evolution. The advantage of the fighting fish is that the fighting continues for more than one hound sometimes up to 2 hours, facilitating us to analyze the transcriptome of fighting. Accordingly, in the case of fighting of fighting fish, the new transcriptome that characterizes fighting appears during fighting, which is so-called the neurogenomic state of fighting. According to our preliminary analysis of RNA-Seq of the transcriptome, there are many transcripts that show the difference by 4 folds expression between before fighting and during fighting. About after 20 mins of fighting, IEGs (immediate Early Genes) expression can be observed. These expressions are believed to be needed for consolidation of memory. Therefore, this is a very good system to analyze what kind of gene expressions are required to consolidation of memory of fighting.
3A HAYASHI, Masafumi ${ }^{1}$, KEYAMURA, Kenji ${ }^{1}$, -01 HISHIDA, Takashi ${ }^{1}\left({ }^{1}\right.$ Dept. Life Sci., Grad. Sch. Sci., Gakushuin Univ.)

Identification of phosphorylation-dependent regulatory mechanism for DNA damage tolerance in Saccharomyces cerevisiae

DNA Damage Tolerance (DDT) pathway, comprises many of the ubiquitin conjugation factor, promotes mono- and polyubiquitination of proliferating-cell nuclear antigen, which facilitates bypass of lesions by replicating across the damaged template through the error-prone DNA polymerases or using the undamaged sister chromatid as a template. These ensure the completion of replication without removing the lesions, therefore enabling cells to repair them at a later time. Thus, DDT pathway plays an important role in the maintenance of genome integrity during replication. In this study, we expressed epitope-tagged DDT proteins and examined phosphorylation status of them in the presence or absence of DNA damage. Using Phos-tag SDS-PAGE, we found that Rad5 was detected as at least two distinct bands, and slower-migrating bands were disappeared after treatment with lambda phosphatase. We also showed that Rad5 phosphorylation was not enhanced by DNA damage, but become more prominent in the S/G2/M phase cells than in G1 cells. These results suggest that Rad5 is phosphorylated in cell-cycle dependent manner, although a mechanism that is at present unclear.

3A MASUDA, Yuji ${ }^{1,2}$, KANAO, Rie $^{1}$, MASUTANI, -02 Chikahide ${ }^{1}\left({ }^{1}\right.$ Dept. Genome Dynamics, Res. Inst. of Env. Medicine, Nagoya Univ., ${ }^{2}$ Dept. Toxicogenomics, Nagoya Univ. Grad. Sch. Med.)

Biochemical analysis of HLTF, a human homologue of SWI/ SNF-related ubiquitin ligase RAD5

DNA is constantly damaged by a wide variety of exogenous and endogenous agents and most of such DNA lesions inhibit DNA synthesis. To cope with such problems during replication, cells have molecular mechanisms to continue DNA replication in the presence of DNA lesions. Such DNA damage tolerance mechanisms are divided into two pathways, translesion DNA synthesis and template switch (TS), and they are activated by mono- and poly-ubiquitination of PCNA, respectively. In yeast, RAD5, a ubiquitin ligase, plays a role for the poly-ubiquitination of PCNA, and it has been believed that HLTF, a human homologue of RAD5, has the equivalent function in humans. However, molecular mechanisms for the stimulation of RAD5/HLTF at the damage site under the DNA replication remain obscure. We found previously that the ligase activity of HLTF is DNAdependent. Here, we report biochemical data about the ligase activity of HLTF and discuss regulatory mechanisms of HLTF. 
3A NISHIDA, Hirokazu ${ }^{1,2}$, TAKUYA, Yoda ${ }^{3}$, TANABE, -03 Maiko ${ }^{2}$, TSUJI, Toshiyuki ${ }^{4}$, YODA, Takao ${ }^{4}$, SHIRAI, Tsuyoshi $^{4}$, TAKEYAMA, Haruko ${ }^{3}$, ISHINO, Yoshizumi $^{5} \quad\left({ }^{1}\right.$ New Energy and Ind. Tech. Dev. Organization, ${ }^{2}$ Hitachi, Ltd. Res. \& Dev. Group, ${ }^{3}$ Dept. Life Sci. and Med. Biosci., Waseda Univ., ${ }^{4}$ Dept. Comp. BioSci., Nagahama Inst. Bio-Sci. Tech., ${ }^{5}$ Dept. BioSci. and BioTech., Faclt. Agr., Kyushu Univ.)

Exonuclease processivity of archaeal replicative DNA polymerase in association with PCNA is expedited by mismatches in DNA

Replicative DNA polymerase comprises two independent domains, Polymerase (DNA-synthesis) and Exonuclease (DNAcorrection (degradation)) domains, and switches the DNA interacting domain by altering the relative arrangement to PCNA molecule.

In the two DNA polymerase/PCNA/DNA complex structures in polymerase and exonuclease modes, PCNA seems to hold the DNA substrate, whereas the DNA polymerase dynamically alters its relative arrangement to the others. In this study, we constructed several alanine-substituted mutants of the basic residues inside the PCNA ring presumably responsible for the DNA interaction, and examined the polymerase and exonuclease reactions performed by DNA polymerase with PCNA. As a result, the alanine-substituted mutants of PCNA exhibited the substantial enhancement only in the exonuclease reaction. On the other hand, when the mismatch-induced DNA were employed for the substrates, in which the PCNA-DNA interactions are not expected to be retained, the exonuclease reaction was facilitated. Taken together, PCNA-DNA interaction formed between the basic residues inside PCNA and normal dsDNA may act as the suppressor of the exonuclease reaction.

3A TAKATO, Yokoi ${ }^{1}$, KOJIMA, Yuna ${ }^{1}$, TANAKA, -04 Shuitsu ${ }^{1}$, HATAKEYAMA, Shin ${ }^{1}\left({ }^{1}\right.$ Lab. Genet., Grad. Sch. Sci. Eng., Saitama Univ.)

Function of Neurospora crassa MSH1 in the maintenance of mitochondria DNA

The MutS protein, which recognizes the misincorporated nucleotide in the newly synthesized DNA strand in E. coli, is also found in eukaryotic cells as MSH (MutS homolog) proteins, MSH1 to MSH6. Among these, MSH1 is conserved exclusively in fungi, so this protein implied to have unique role in fungal cell. Knockout of the msh1 gene in Neurospora caused severe phenotypes, such as early cease of hyphal growth, and showing mutagen sensitivity and deletion of mitochondrial DNA (mtDNA). By fusing with the GFP, MSH1 protein revealed its localization in mitochondria. Further, endogenic expression of this protein without mitochondrial localization signal, which found in the N-terminus of Neurospora MSH1 polypeptide, showed similar phenotypes to that of the msh 1 knock out mutant. These results indicate that the MSH1 protein may function in the maintenance of mtDNA which is indispensable to sustain mitochondrial function one of which correlates to energy supply for the elongation of hyphal tip.
3A MIYAJI, Masahiro $^{1}$, KATO, Yuichi ${ }^{1}$, HAYASHI, -05 Yuichiro ${ }^{1}$, ZHANG-AKIYAMA, Qiu-mei ${ }^{1}$ ( ${ }^{1}$ Div. Biol. Sci., Grad. Sch. Sci., Kyoto Univ.)

Phenotypic analysis of base excision repair deficient nematode

To be submitted later

3A TAKANO, Noriko ${ }^{1}$, OHNO, Mizuki ${ }^{1}$, SASAKI, -06 Fumiko ${ }^{1}$, NAKATSU, Yoshimichi ${ }^{1}$, TSUZUKI, Teruhisa ${ }^{1}$ ( ${ }^{1}$ Dept. Med. Biophys. and Radiat. Biol., Faclt. Med. Sci., Kyushu Univ.)

Mismatch DNA repair system effectively maintain the integrity of genome in somatic and germline cells

Mismatch DNA repair (MMR) system is required for the accurate DNA replication. Deficiency of MMR leads to a hyper mutator phenotype and consequently increases a tumor incidence. MMRdefect is well known as the cause of the hereditary colorectal cancer in human.

To clarify the role of MMR in mammalian germline mutation rate, we analyzed de novo germline mutations by whole exome sequencing using parent-child-trio sample of $M s h 2$ deficient mice. We found an increased incidence of indel mutations of 1-2 units in tandem repeats (TR) in addition to single base substitution mutations. These results indicate that MMR play an important role in the maintenance of genome integrity of germline cells in mice. It suggests the efficiency of MMR directly influences to the rate of genetic diversity. 
3A HAYASHIDA, Genki ${ }^{1}$, NAKATSU, Yoshimichi ${ }^{2}$, -07 HIDAKA, Kyoko ${ }^{3}$, FUJIKANE, Ryosuke ${ }^{4}$, HIDAKA, Masumi $^{4}$, TSURIMOTO, Toshiki ${ }^{5}$, TSUZUKI, Teruhisa ${ }^{2}\left({ }^{1} \mathrm{Grad}\right.$. Sch. of Systems of Life Sci.s, Kyushu Univ., ${ }^{2}$ Dept. Med. Biophys. and Radiat. Biol., Faclt. Med. Sci., Kyushu Univ., ${ }^{3}$ Cent. Fundamental Educ., KitaKyushu Univ., ${ }^{4}$ Dept. Odontology, Fukuoka Dental Coll., ${ }^{5}$ Dept. Biol., Faclt. Sci.s, Kyushu Univ.)

Isolation of mismatch repair factor MSH2 variants found in Lynch syndrome with CRISPR/Cas9 system

DNA mismatch repair (MMR) system maintains genome integrity by correcting replication errors. MMR is also known to involve in apoptotic responses to DNA damaging agents. Germline mutations in MMR genes cause hereditary nonpolyposis colorectal cancer (Lynch syndrome). The somatic mutations also cause sporadic colorectal cancers. In mice, a mutation in ATPase domain, G674A, is reported to cause deficiency in MMR but not in apoptotic induction, suggesting that the induction of apoptosis is independent from the repair reaction. We developed a new system to characterize MSH2 variants found in Lynch syndrome patients using the Epstein-Barr virus-based expression vector. Exogenously expressed G674A mutant protein (G674A) successfully interacted with its endogenous partner, MSH6. The resulting mutant MutS? could bind DNA with G/T mismatch, but failed to form a sliding clamp on DNA. Contrary to the results of G674A knock-in mutant mice, HeLa cells expressing G674A were deficient in apoptosis induction. In order to confirm these results, we isolated mutant HeLa cell lines harboring G674 mutations using genome editing with CRISPR/Cas9 system, and currently characterize these cell lines.

3A SHIOIRI, Takuma ${ }^{1}$, KEYAMURA, Kenji ${ }^{1}$, HISHIDA, -08 Takashi ${ }^{1}\left({ }^{1}\right.$ Dept. Life Sci., Grad. Sch. Sci., Gakushuin Univ.)

Mechanism of cellular tolerance to chronic low-dose UV irradiation in nucleotide excision repair-deficient cells in Saccharomyces cerevisiae

Genome DNA is constantly damage by exogenous and endogenous DNA damaging agents. The major source of exogenous DNA damage is ultraviolet (UV) radiation present in sunlight. It causes replication-blocking lesions such as pyrimidine dimers, which are primarily repaired by the nucleotide excision repair (NER) pathway. Our previous studies demonstrated that while chronic low-dose UV light (CLUV) does not impair the growth of NER-deficient rad14A yeast cells during 16 hours, despite the observation that DNA lesions continued to accumulate with CLUV exposure. In this study, rad14A cells were grown for several days by diluting the cultures into fresh medium every 24 hours under CLUV irradiation. The result shows that long-term exposure to CLUV induces homologous recombination to confer CLUV-resistant phenotype in rad14 $\Delta$ cells. These results suggest that the homologous recombination pathway is important to genomic integrity and survival in mitotically growing cell when irreparable DNA damages are accumulated.
3A FUNAKOSHI, Masafumi ${ }^{1}$, MATSUDA, Ryousuke ${ }^{1}$, -09 ZHANG-AKIYAMA, Qiu-Mei ${ }^{1}{ }^{1}$ Stress Response Biol., Div. Biol. Sci., Grad. Sch. Sci., Kyoto Univ.)

The role of Ciona intestinalis' AP endonuclease, CiAPEX1, CiAPEX2 and CiP0 in AP site repair

In this study, we focused on Apurinic/apyrimidinic (AP) sites as DNA damages which induce genomic instability. AP sites are well-known DNA damages, this is because these damages are most frequently generated in living body and AP sites accumulation are known to induce severe effects to organisms. AP endonuclease play an important role in AP site repair and three types of AP endonuclease have been reported, APEX1, APEX2 and P0. APEX1 is the major AP endonuclease and repair almost all the AP sites in mammals. On the other hand, APEX2 and P0 have unique characteristics. APEX2 are expected to contribute to the mitochondrial genome integrity and $\mathrm{P} 0$ is a ribosomal protein. However it is not known why organisms have AP endonuclease more than one and how these AP endonucleases contribute to the genome integrity. In this study, to solve these problems, we compare the function of all the AP endonuclease in Ciona intestinalis, which is a model organism in developmental biology.

3A LE, Lan Anh Thi ${ }^{1}$, MAKI, Hisaji ${ }^{1}\left({ }^{1}\right.$ Lab. Microb. -10 Molec. Genet., Draduate Sch. Biol. Sci.s, Nara Inst. Sci. Tech.)

Oxidative DNA damage is a major cause of spontaneous occurrence of homologous DNA recombination

Reactive oxygen species are a major cause of spontaneous mutations in aerobic organisms by attacking DNA to produce many kinds of oxidative DNA damages which induce various mutations and chromosomal aberrations. Recently, our laboratory found that environmental factors such as nutrient, oxygen concentration and $\mathrm{pH}$ have a significant effect on cellular level of oxidative DNA damages. However, it was also shown that spontaneous base substitution mutations are not affected by such environmental factors. Here we show that the RecAdependent chromosomal rearrangements are affected by the environmental factors which vary the cellular level of oxidative DNA damages. In order to investigate spontaneous recombination events, a strain containing two copies of rpsL genes was constructed, and allelic recombination events between two rpsL genes were detemined. The result shows that environemtal factors promoted spontaneous recombination events through the production of oxidative DNA damages. 
ZHANG, Yingbiao ${ }^{1}$, MATSUZAKA, Tomoyuki ${ }^{2}$, YANO, Hirokazu ${ }^{3}$, NAKANO, Toshiaki ${ }^{4}$, ISHIKAWA, Ken ${ }^{5}$, FUKUYO, Masaki ${ }^{6}$, TAKAHASHI, Noriko ${ }^{7}$, SUZUKI, Yutaka ${ }^{8}$, SUGANO, Sumio $^{8}$, IDE, Hiroshi ${ }^{9}$, KOBAYASHI, Ichizo ${ }^{10}\left({ }^{1}\right.$ Univ. Tokyo, ${ }^{2}$ Dept. Math and Life Sci.s, Grad. Sch. Sci., Hiroshima Univ., ${ }^{3}$ Dept. Comput. Biol. and Med. Sci. (formerly Dept. Med. Genome Sci.), Grad. Sch. Front. Sci., Univ. of Tokyo, ${ }^{4}$ Dept. Math. and Life Sci.s, Grad. Sch. Sci., Hiroshima Univ., ${ }^{5}$ Natl. Cancer Inst., Natl. Inst. Health, ${ }^{6}$ Dept. Mol Oncol., Grad. Sch. Med., Chiba Univ., ${ }^{7}$ Dept. Comput. Biol. and Med. Sci. (formerly Dept. Med. Genome Sci.), Grad. Sch. Front. Sci.s, Univ. of Tokyo, ${ }^{8}$ Dept. Comput. Biol. and Med. Sci. (formerly Dept. Med. Genome Sci.), Grad. Sch. Front. Sci.s, Univ. of Tokyo, ${ }^{9}$ Dept. Comput. Biol. and Med. Sci. (formerly Dept. Med. Genome Sci.s), Grad. Sch. Front. Sci., Univ. Tokyo, ${ }^{10}$ Dept. Math. and Life Sci.s, Grad. Sch. Sci., Hiroshima Univ., ${ }^{11}$ Dept. Comput. Biol. and Med. Sci. (formerly Dept. Med. Genome Sci.), Grad. Sch. Front. Sci., Univ. Tokyo)

Restriction glycosylases: involvement of endonuclease activities in the restriction process

All the examined restriction enzymes were phosphodiesterases generating 3' $\mathrm{OH}$ and 5' $\mathrm{P}$ ends, but a restriction enzyme (restriction glycosylase), R.PabI from a hyperthermophile, ascribed the breakage to high temperature while the other report identified its weak AP lyase activity generating atypical ends. Here we addressed this issue in mesophiles. We first purified R.PabI homologs from Campylobacter coli (R.CcoLI) and Helicobacter pylori (R.HpyAXII) and demonstrated their DNA cleavage, DNA glycosylase and AP lyase activities in vitro at $37 \mathrm{oC}$. AP lyase is more coupled with glycosylase in R.CcoLI than in R.PabI. R.CcoLI or R.PabI expression caused restriction of incoming bacteriophage/plasmid DNA and endogenous chromosomal DNA within E. coli at 37oC. The R.PabI-mediated restriction was promoted by AP endonuclease action in vivo or in vitro. These results revealed the role of endonuleolytic DNA breakage in the restriction and yet diversity in the endonucleases involved. The cleaved ends are difficult to repair in vivo, which may signify their biological significance. Such difficulty may be useful in application.

3A HAYASHI, Yuichiro $^{1}$, QIU-MEI, Zhang-Akiyama ${ }^{1}$ -12 ( ${ }^{1}$ Lab. Stress Response Biol., Grad. Sch. Sci., Kyoto Univ.)

The involvement in genomic stability maintenance of KsgA, DNA glycosylase in E.coli, and its human homologs

Base Excision Repair (BER) is a system repairing damaged bases. DNA glycosylase works at the first step in BER. Once, we revealed that KsgA, a dimethyltransferase in E.coli, is a bifunctional DNA glycosylase removing cytosine paired with thymineglycol and 5-formyluracil. We also elucidated that KsgA suppresses spontaneous mutation frequency. Furthermore, we confirmed that human homologs of KsgA, DIMT1L and TFB1M, have DNA glycosylase and AP lyase activities and suppress spontaneous mutation frequency as well as KsgA. We research the involvement in maintenance of genome stability of KsgA and its human homologs now.
3B YUKUHIRO, Kenji ${ }^{1}$, SAKAGUCHI, Hiroki ${ }^{2}$, ITOH, -01 Masanobu ${ }^{2}$, KOMOTO, Natuo ${ }^{1}$, TOMITA, Shuichiro ${ }^{1}$ $\left({ }^{1} \mathrm{NARO}\right.$, Inst. AgroBiol. Sci., ${ }^{2}$ Kyoto Inst. Tech.)

Genetic differentiation between Japanese population of the mulberry silkworm, Bombyx mandarina by the biased distribution of three SNPs on COI gene

Three out of 135 SNP sites (SNP156, 325 and 666) on COI gene played a crucial role in dividing Japanese populations of Bombyx mandarina. In SNP325, three variable forms were observed. Two of them are highly frequent and distribute biasedly: SNP325T is fixed in populations present in districts west to Hyogo prefecture. Populations in Hyogo as well as two northern Kyoto populations show polymorphic state of both forms. In eastern populations, SNP325C were fixed or nearly fixed except for some populations in Kanto districts. These populations included variable levels of SNP325T, especially SNP325T is fixed in Kimitsu population. SNP156T is predominated in 10 populations in the northern part of Japan, including Hakodate, Hokkaido to Tsuruoka-Asahi, Yamagata prefecture. High frequencies of SNP666A are limitedly detected in two populations, Daisen, Tottori prefecture and GozuIzumo, Shimane prefecture. SNP666A and SNP666G are segregated in two populations, Takahashi-Makabe, Okayama prefecture, and Mihara, Hiroshima prefecture. It is notable that these three sites are fixed in Bombyx mori, Chinese B. mandarina and B. huttoni.

3B MORIGUCHI, Natsuki ${ }^{1}$, UCHIYAMA, Kentaro ${ }^{2}$, -02 MIYAGI, Ryutaro ${ }^{3}$, TAKAHASHI, $\mathrm{Aya}^{3}$, TAMURA, Koichiro $^{3}$, TSUMURA, Yoshihiko ${ }^{4}$, TESHIMA, Kosuke M. $^{6}$, KUSUMI, Junko ${ }^{5}$ TACHIDA, Hidenori ${ }^{6}$ ( ${ }^{1}$ Evolutionary Genet. Lab., Dept. Systems Life Sci.s, Kyushu Univ., ${ }^{2}$ Dept. Forest Genet., Forestry Forest Products Res. Inst., ${ }^{3}$ Evolutional Genetic Lab., Dept. Biol. Sci.s, Tokyo Metro. Univ., ${ }^{4}$ Faclt. Life and Env. Sci.s, Univ. Tsukuba, ${ }^{5}$ Dept. Env. Changes Faclt. Social and Cultural Studies, Kyushu Univ., ${ }^{6}$ Dept. Biol., Faclt. Sci.s, Kyushu Univ.)

Analysis of population structure of Cryptomeria japonica based on amplicon sequenced data

Cryptomeria japonica is a coniferous tree species with a broad natural distribution in the Japanese archipelago. There are two varieties distinguished by their morphological traits: the ura-sugi variety (C. japonica var. radicans) has slender branchlets with soft leaves and is mainly distributed on the Japan Sea side, while the omote-sugi variety (C. japonica) has rough branchlets with hard leaves and is distributed on the Pacific Ocean side. Because each variety consists of two groups of populations, C. japonica comprises 4 groups of populations. In this study, 94 samples from 4 natural populations (Ajigasawa, Bijodaira, Shimowada, Yakushima), each representing one of the 4 groups, were used to determine sequences at 142 nuclear genes using next generation sequencing. Three clusters were identified by a PCA analysis, Yakushima, Omote-sugi and Ura-sugi clusters. The phylogenic tree estimated by software TreeMix suggested the Yakushima population first diverged from the other populations and later the Ura-sugi populations originated from the Omote-sugi population. An application of software BayeScan to the 142 genes data revealed no candidate genes under natural selection. 
3B KAWAMURA, Sayu ${ }^{1}$, TESHIMA, Kosuke ${ }^{1}$ ( ${ }^{1}$ Dept. -03 Biol., Faclt. Sci.s, Kyushu Univ.)

Study of population differentiation measures

$F_{S T}$ is one of the most widely used measurements to assess genetic differentiations. Many researchers arranged calculation methods and proposed relating measures. To understand the properties of these $F_{S T}$ related measures, we conducted coalescent simulation, and calculated $F_{S T}$-related measures. As genetic markers, DNA sequences, SNPs and microsatellites were considered.

Judging from the pattern of the values, the measures were classified into three groups. This classification agrees with the grouping taken into account their definitions: measures divided by between-population-diversity, divided by total-diversity, and others. It was also found that measures in the same group converged to the same value regardless of marker types as long as we can use enough markers. We also evaluated the effect of the ascertainment bias on the distribution of $F_{S T}$ calculated from SNP typing data. The effects of the ascertainment appeared as the shift of the distributions, but the direction of the shift depends on the types of ascertainment schemes for SNP discovery processes. We should be careful about the calculation method of measures and the ascertainment bias when we use genotyping data.
3B HARADA, Ko ${ }^{1}$, DWIYANTI, Fifi Gus ${ }^{1}$, KAMIYA, -05 Koichi ${ }^{1}$, SIREGAR, Iskandar ${ }^{1}$, DEWAY, Bibian ${ }^{1}$, CHONG, Lucy ${ }^{1}$ ( ${ }^{1}$ Faclt. Agr., Ehime Univ.)

Phylogeography of Dryobalanops aromatica (Dipterocarpaceae) in Sundaic region

Two hundreds and twenty three Leaf samples of Dryobalanops aromatica (Dipterocarpaceae) were collected from 9 populations in the tropical rain forests of Sundaic region in Southeast Asia. Genetic variation was examined by using 8 microsatellite loci. The Bayesian model based cluster analysis showed the best number of $\mathrm{K}=2$ indicating these populations were grouped into two in Sumatra-Malay and Borneo. By using IM (Isolation with Migration) simulation, these groups were found to be separated 7200 years ago. It was also shown that the ancestral population was twenty times larger than the present populations as a whole. These suggest the tropical rain forest was expanded much larger than today's at the last glacial maximum (LGM), which occurred twenty thousand years ago. Considerable amount of migrations between the two groups also suggests that the hypothetical savanna corridor on the center of the exposed Sunda Shelf at the LGM was disconnected by forest belts.

3B YASHIMA, Akiko Sato ${ }^{2}$, INNAN, Hideki ${ }^{1}\left({ }^{1}\right.$ Dept. -06 Math. Eng., Musashino Univ., ${ }^{2}$ Dept. Evolutionary Studies of Biosyst., Sch. Adv. Sci., The Grad. Univ. Adv. Stud. (SOKENDAI))

Genetic Variations of the marine fishes and the freshwater fishes

Understanding the patterns of genetic variation within and between populations is important in ecology, evolution, conservation and population genetics. Although tremendous amounts of effort have been made to evaluate microsatellite variation in natural populations for over 5,000 species so far, there have been very few works that compared multiple species. We analyzed microsatellite variation in more than 200 fish species to explore what determine the species-wide pattern of genetic variation. We found that their migration ability and preference (e.g., freshwater vs marine environment) is the major factor to specify the pattern of genetic variation. 
3B OKA, Ayako ${ }^{1}$, SHIROISHI, Toshihiko ${ }^{1}\left({ }^{1}\right.$ Mammalian -07 Genet. Lab., Natl. Inst. Genet.)

Polymorphism in the cis-regulatory elements and its impact on the reproductive isolation

Improper gene regulation is implicated in reproductive isolation, but its genetic bases are unknown. We previously reported that mouse inter-subspecific X chromosome substitution strains (B6$\mathrm{ChrX} \mathrm{XSM}^{\mathrm{MSM}}$ and $\mathrm{B} 6-\mathrm{XT}^{\mathrm{MSM}}$ ) show reproductive isolation characterized by male-specific sterility due to disruption of meiotic entry during the spermatogenesis. Transcriptome analysis of these strains revealed that a gross aberration in gene expression on the substituted $\mathrm{X}$ chromosome in testis. The misregulation of $\mathrm{X}$ linked genes showed asymmetry; more genes were disproportionally downregulated rather than upregulated. This suggests that genetic incompatibility between cis-regulatory elements and trans-acting regulatory factors may occur in the transcriptional regulation of genes on the substituted X chromosome. To clarify the genetic basis of cis-trans incompatibility, we identified enhancers for $T a f 7 l$ gene that is a candidate gene responsible for the small testes of $\mathrm{B} 6-\mathrm{XT}^{\mathrm{MSM}}$ strain. The result from the luciferase assay showed allelic differences of enhancer activities.

3B MATSUMOTO, Yuki ${ }^{1,2,3}$, NISHINO, Jo ${ }^{4}$, NAKAOKA, -08 Hirofumi $^{5}$, GOTO, Tatsuhiko ${ }^{6}$, KOIDE, Tsuyoshi ${ }^{1,2}$ $\left({ }^{1}\right.$ Grad. Sch. Life Sci., SOKENDAI, ${ }^{2}$ Mouse Genomics Resource Lab., Natl. Inst. Genet., ${ }^{3}$ JSPS Res. Fellow, ${ }^{4}$ Grad. Sch. Med., Nagoya Univ., ${ }^{5}$ Div. Hum. Genet., Natl. Inst. Genet., ${ }^{6}$ Sch. Life Sci., The Univ. of Nottingham)

Identification of genetic loci associated with tameness using selection and association mapping in mice

Tame behavior is one of the major elements in domestication and defined as increased interaction of animals with human. To identify genes associated with active tameness which is defined as contacting human hand (contacting), we performed selective breeding for contacting using wild-derived heterogeneous stock (WHS), which is a mixed population derived from 8 wild mouse strains originated in various geographic regions. Following the selective breeding, we used selection mapping as well as association mapping and found two candidate loci on Chromosome 11 potentially associated with contacting.
3B GONDO, Yoichi $^{1}$, FUKUMURA, Ryutaro ${ }^{1}$, -09 ISHITSUKA, Yuichi ${ }^{1}$, MAKINO, Shigeru ${ }^{1}$, MORI, Kazuki $^{2}$, KUHARA, Satoru ${ }^{2}$, TOYODA, Atsushi ${ }^{3}$, FUJIYAMA, $\mathrm{Asao}^{3}{ }^{1}$ RIKEN BioRes. Cent., ${ }^{2}$ Faclt. Agr., Kyushu Univ., ${ }^{3}$ Cent. Inform. Biol., Natl. Inst. Genet.)

Detection and analysis of spontaneous mutations in standard inbred mice: whole genome sequencing (WGS) and single nucleotide variation (SNV) call

We have accumulated spontaneous mutations in C57BL/6JJcl inbred mice for 4 generations by complete outbreeding method. We have generated 8 independent pedigrees, the fourth-generation females of which were subjected to WGS and SNV calls. The mutations were accumulated in the complete outbreeding scheme so that de novo mutations should be found only in one fourthgeneration female as heterozygotes. Within non-repetitive genomic sequences with sufficient read coverage, a total of 17,325 SNV candidates were primarily called. Among them, 1,874 SNV were homozygous commonly found in all the eight fourthgeneration females; thus, they are fixed SNPs. The number of unique heterozygous SNVs found in only one of the 8 fourthgeneration females was 2,069 that encompass the de novo mutations. Seven hundreds and twenty one candidates were un-fixed SNPs. Peculiarly, the remaining 12,661 candidates were found in all the 8 fourth-generation females as heterozygotes. Such a large number of false-positive SNV calls strongly indicate some problems of the current mouse genome reference sequence like segmental duplications.

3B FUKUMURA, Ryutaro ${ }^{1}$, KOTAKI, Hayato $^{1}$, -10 ISHITSUKA, Yuichi ${ }^{1}$, MAKINO, Shigeru ${ }^{1}$, NAKAI, Yuji $^{1}$, MORI, Kazuki ${ }^{2}$, KUHARA, Satoru ${ }^{2}$, TOYODA, Atsushi $^{3}$, FUJIYAMA, Asao ${ }^{3}$, GONDO, Yoichi ${ }^{1}$ $\left({ }^{1}\right.$ RIKEN BioRes. Cent., ${ }^{2}$ Faclt. Agr., Kyushu Univ., ${ }^{3}$ Cent. Inform. Biol., Natl. Inst. Genet.)

Detection and analysis of spontaneous mutations in standard inbred mice: validation and tracing of SNV (single nucleotide variation) candidates

We have extracted 2,069 SNV candidates by WGS from eight G5 female genomes in which independent spontaneous mutations had been accumulated for 4 generations (from G1 to G5 generation) with no selection to recessive deleterious mutations (Gondo et al. This meeting). We have validated 1,649 SNV candidates from 6 out of the 8 genomes and 1329 have so far been experimentally confirmed to be true mutations. Among them, 1074 SNV were also found in a few of the eight G1 mice; thus, they are newly arisen rare SNPs in the breeder's colony. The remaining $255 \mathrm{SNV}$ were de novo mutations during the four generations. Roughly three quarters of the mutations occurred on GC pairs. By tracing 23 confirmed de novo mutations in the pedigrees, 6, 8, 6 and 3 were derived from G2, G3, G4 and G5 mice, respectively by Mendelian inheritance. It was completed to accumulate spontaneous mutations for four generations in a year in the mouse. This mutation analysis scheme is universally applicable to any species that proliferate by sexual reproduction. 
3B OSADA, Naoki ${ }^{3}$, MIYAGI, Ryutaro ${ }^{1}$, TAKAHASHI, -11 Aya $^{1,2}\left({ }^{1}\right.$ Dept. Biol. Sci.s, Tokyo Metro. Univ., ${ }^{2}$ Res. Cent. Genomics and Bioinform., Tokyo Metropolitan Univ., ${ }^{3}$ Grad. Sch. Inform. Sci. Tech., Hokkaido Univ.)

Genome-wide analysis of cis- and trans-regulatory effects on gene expression using allele samples from a natural population of Drosophila melanogaster

Quantifying the relative contributions of cis- and trans-regulatory variations on intraspecific gene expression is an essential step to understanding the evolution of transcriptome. We quantified the genome-wide allele specific expression (ASE) variations in F1s between 18 different Drosophila melanogaster strains sampled from the Drosophila Genetic Reference Panel (DGRP) and a reference strain from another population. Head and body samples of the F1 adult females were subjected to RNAseq and the subsequent ASE quantification. A linear model approach revealed that the degree of relative cis-regulatory contribution on gene expression variation is affected most strongly by local polymorphism level in both head and body samples. The degree of relative trans-regulatory contribution was strongly affected by local recombination rate in head and by female-bias in expression in body. Analysis of the effects of proximal transposon element (TE) insertions on ASE revealed that TE insertions affected transcription levels of ovary-expressed genes more pronouncedly than genes that are not expressed in ovary.

3B TAKADA, Yasuaki ${ }^{1}$, TAKAHASHI, Aya ${ }^{2}$, MIYAGI, -12 Ryutaro ${ }^{2}$, ENDO, Toshinori ${ }^{1}$, OSADA, Naoki ${ }^{1}\left({ }^{1} \mathrm{Grad}\right.$. Sch. Inform. Sci. Tech., Hokkaido Univ., ${ }^{2}$ Dept. Biol. Sci.s, Tokyo Metro. Univ.)

The cause of gene expression changes in reciprocal crosses of Drosophila melanogaster by measuring allele-specific gene expression

Elucidating how gene expression is regulated by intercellular signals is an important subject in genetics. There are three major factors affecting the allele-specific gene expression (ASE), cisregulatory effect, imprinting effect and maternal effect. However, methods to evaluate these three effects has not been established because the three factors are highly confounding. Here, we propose a method to evaluate these effects on the ASE and decomposing these factors. We estimated the ASE from RNA-seq data obtained from reciprocally-crossed adult fruit flies (Drosophila melanogaster), and applied the new method. As a result, only the cis-regulatory effect were detected and there was no detectable imprinting and maternal effect in adult flies. In contrast, analysis of mouse trophoblast stem cells showed that the cis-regulatory effect was the strongest, the imprinting effect was the next, and the maternal effect was the weakest in mice.
3C NAKAGAWA, Takuro ${ }^{1}$, ONAKA, Atsushi ${ }^{1}$, -01 TAKAHASHI, Tatsuro S. ${ }^{1}$, MASUKATA, Hisao ${ }^{1}$ $\left({ }^{1}\right.$ Dept. Biol. Sci.s, Grad. Sch. Sci., Osaka Univ.)

Rad51 and Rad54 promote noncrossover recombination between centromere repeats to prevent isochromosome formation

Non-allelic recombination between repetitive DNA elements in the genome can result in gross chromosomal rearrangements (GCRs). Our previous studies have shown that Rad51 suppresses the formation of isochromosomes that are produced by recombination between inverted repeats in the centromere that is essential for proper segregation of chromosomes. However, it remains unclear how Rad51 suppresses such homology-mediated GCRs. Here, we found in fission yeast that Rad51 and Rad54, which binds and regulates Rad51, promote a noncrossover type of recombination between centromere repeats, thereby suppressing crossover recombination that leads to the isochromosome formation. Mutations in Rad51 and Rad54 severely reduced noncrossover recombination between inverted repeats in the centromere, and greatly increased the rate of the isochromosome formation. Deletion of Mus81 endonuclease that is required for crossover recombination reduced both crossovers between inverted repeats in the centromere (i.e. inversions) and isochromosomes. Thus, we propose that homologous recombination catalyzed by Rad51 and Rad54 preferentially promote noncrossover thereby suppressing homology-mediated GCRs.

3C ARAKI, Masatake ${ }^{1}$, TAKEDA, Iyo $^{1}$, OHGA, -02 Toshinori ${ }^{1}$, ETO, Satoshi ${ }^{1}$, NAKAHARA, Mai ${ }^{1}$, YOSHINOBU, Kumiko ${ }^{1}$, ARAKI, Kimi ${ }^{1}$ ( ${ }^{1}$ Inst. Resource Dev. and Anal., Kumamoto Univ.)

Functional analysis of chromosome specific clustered trap region (CSCT) 13

We developed the Database for the Exchangeable Gene Trap Clones (EGTC) (http://egtc.jp). During the annotation of trap clones, we found new genome element CSCT (Chromosome Specific Clustered Trap region). There were 39 EGTC clones mapped in the CSCT region, they were distinguished in CSCT2, CSCT4, CSCT12 or CSCT13. Using CRISPR/Cas9 system, CSCT13 region $(1.6 \mathrm{Mbp})$ was deleted in the mouse ES cells (CSCT KO). We could establish CSCT KO mouse line. Mating between heterozygotes gave apparently normal homozygotes. However, mating between homozygotes gave relatively small number of pups. Moreover, CSCT13 KO mice showed comparatively low rate of homologous recombination during meiosis for the region corresponding to CSCT13. On the other hand, outside of this region showed up-regulation of homologous recombination during meiosis. This study suggest that CSCT13 might be related with the early embryogenesis and homologous recombination during meiosis. 
3C OHGA, Toshinori ${ }^{1}$, TAKEDA, Iyo ${ }^{1}$, ETO, Satoshi ${ }^{1}$, -03 NAKAHARA, $\mathrm{Mai}^{1}$, YOSHINOBU, Kumiko ${ }^{1}$, ARAKI, Kimi $^{1}$, ARAKI, Masatake ${ }^{1}\left({ }^{1}\right.$ Inst. Resource Dev. and Anal., Kumamoto Univ.)

Establishment of a mouse line deleted CSCT2 region (3.1 $\mathrm{Mbp}$ )

We found new genome element CSCT (Chromosome Specific Clustered Trap region), and studied CSCT13 region exist in chromosome 13. In the analysis of CSCT13 region, we are obtaining interesting results suggesting the important role in the early embryogenesis and homologous recombination during meiosis. So this time, we focused on the CSCT2 region (present in chromosome 2) and decided to produce a knockout mouse line to know if this region has similar function with CSCT13, or not. By using the knock-in vector comprising a left arm (1.7kbp) and a right arm $(1.3 \mathrm{kbp})$, and pX459 to express puro transiently, it was deleted the entire region $(3.1 \mathrm{Mbp})$. It's efficiency was approximately $15 \%$. We could establish mouse lines from the ES cells and have started phenotype analysis.

3C MOURI, Kousuke ${ }^{1}$, SAGAI, Tomoko ${ }^{1}$, AMANO, -04 Takanori $^{1}$, SHIROISHI, Toshihiko ${ }^{1}$ ( ${ }^{1}$ Natl. Inst. Genet.)

Enhancer rearrangement causes regulatory change of gene expression

Genomic structural mutation produces new regulation of gene expression via rearrangements of cis regulatory elements. We found that Hammer toe (Hm), a mouse syndactyly mutant, has a $150 \mathrm{~kb}$ translocation from chr14 to chr5, the upstream of Sonic hedgehog (Shh). Though this translocated fragment contained no coding sequences, BAC transgenic reporter assay showed this fragment had an enhancer activity in interdigital region. Ectopic Shh expression in autopod and interdigital region was seen by qPCR.

Syndactyly phenotype of $\mathrm{Hm}$ was not shown when the translocation was placed at a cis-position relative to a Shh knockout allele. It was also not shown when we remove translocated fragment by CRISPR/Cas9. From these results, we concluded that translocated fragment is the cause of syndactyly phenotype by acting as a new enhancer of Shh.

An inversion of translocated fragment which was induced by genome editing enhanced syndactyly phenotype and the level of ectopic Shh expression. On the other hand, Rnf32, which locates at the opposite side of the translocation from Shh, was reduced its ectopic expression by inversion. Translocated fragment is likely to change its activity with its orientation.
3C FUJIMOTO, Akihiro ${ }^{1,2}$, FURUTA, Mayuko ${ }^{2}$, TOTOKI, -05 Yasushi $^{3}$, TSUNODA, Tatsuhiko ${ }^{2}$, KATO, Mamoru ${ }^{3}$, HIROKI, Yamaue ${ }^{4}$, KAZUAKI, Chayama ${ }^{5}$, MIYANO, Satoru $^{6}$, ABURATANI, Hiroyuki ${ }^{7}$, SHIBATA, Tatsuhiro $^{3}$, NAKAGAWA, Hidewaki ${ }^{2}$ ' ${ }^{1}$ Dept. Drug Discovery Med., Grad. Sch. Med., Kyoto Univ., ${ }^{2}$ IMS, RIKEN, ${ }^{3}$ Natl. Cancer Center Res. Inst., ${ }^{4}$ Wakayama Med. Univ., ${ }^{5}$ Hiroshima Univ. Sch. Med., ${ }^{6}$ Inst. Med. Sci., The Univ. Tokyo, ${ }^{7}$ Res. Cent. Adv. Sci. Tech., The Univ. Tokyo)

Whole genome mutational landscape and characterization of non-coding and structural mutations in liver cancer

Liver cancer, which is most often associated with virus infection, is prevalent worldwide, and its underlying etiology and genomic structure are heterogeneous. Here we provide a whole-genome landscape of somatic alterations in 300 liver cancers from Japanese individuals. Our comprehensive analysis identified point mutations, structural variations (STVs), and virus integrations, in noncoding and coding regions. We discovered recurrently mutated coding and noncoding regions, such as long intergenic noncoding RNA genes (NEAT1 and MALAT1), promoters, and regulatory regions. STV analysis found a significant association with replication timing and identified known $(C D K N 2 A, C C N D 1$, $A P C$, and TERT) and new (ASH1L, NCOR1, and MACROD2) cancer-related genes that were recurrently affected by STVs, leading to altered expression. These results emphasize the value of whole-genome sequencing analysis in discovering cancer driver mutations and understanding comprehensive molecular profiles of liver cancer, especially with regard to STVs and noncoding mutations.

3C KRYUKOV, Kirill ${ }^{1}$, IMANISHI, Tadashi ${ }^{1}$ ( ${ }^{1}$ Tokai -06 Univ. Sch. Med., BioMed. Inform. Lab.)

Human Contamination in Genome Assemblies

Contamination in genome assembly can lead to wrong or confusing results when using such genome as reference in sequence comparison. Although the possibility of bacterial contamination is well known, the problem of human-originated contamination received little attention. In this study we systematically surveyed 45,735 available genome assemblies for evidence of human contamination. We determined lineage specificity of individual genome fragments, and used it to distinguish between contamination and conservation. Non-human genome fragments that show high specificity to primates were selected as likely human originated sequences. We found that 154 genome assemblies contain fragments that with high confidence originate as contamination from human DNA. We found that majority of contaminating human sequences were present in the reference human genome assembly for over a decade. We'd like to highlight the importance of contamination detection when performing genome assembly. We recommend that existing contaminated genomes should be revised to remove contaminated sequences, and that new assemblies should be checked for presence of human DNA before submitting them to public databases. 
3C KAMEDA, Masahiro ${ }^{1}$, TESHIGAWARA, Rika $^{1}$, SUN, -07 Liang Tso ${ }^{1}, \mathrm{CHO}$, Junkwon ${ }^{1}$, TADA, Takashi ${ }^{1}\left({ }^{1}\right.$ Dept Stem Cell Eng., Inst. Front. Med. Sci., Kyoto Univ.)

Effect of Anti-aging Hormone ADIPONECTIN on Survival of Human Pluripotent Stem Cell

Human Adiponectin (APN) containing the $\mathrm{C} 1 \mathrm{Q}$ and collagen domains is an adipocyte-secreting protein.

APN functions as a key player of anti-aging in anti-arteriosclerosis, and anti-diabetes.Notably, pluripotent and adult stem cells play an important role in anti-aging by maintenance of homeostasis through adequate replacement of old tissues with regenerated new tissues.However, it remains unknown whether APN is involved in stem cell property. Here, we explored function of APN in survival of human induced pluripotent stem (iPS) cells. Interestingly, survival rate of iPS cells 48 hours after subculture significantly increased by addition of APN in culture medium. Similar results were obtained with different iPS cell lines, and different assay systems.

More detailed analysis demonstrated that effect of APN on improvement of survival rate of iPS cells are obvious in 2-4-cell colonies, but not more than 8-cell colonies, suggesting that APN is involved in survival of iPS cells, prior to establishment of Ecadherin-dependent cell-cell adhesion.

$3 \mathrm{C}$

KOSAKA, Kengo ${ }^{1}$, MIURA, Shiroh ${ }^{2}$, SANO, Ken ${ }^{2}$, -08 FUJIOKA, Ryuta ${ }^{3}$, SAITSU, Hirotomo ${ }^{4}$, TANIWAKI, Takayuki $^{2}$, YAMAMOTO, Ken ${ }^{5}$, SHIBATA, Hiroki ${ }^{1}$ $\left({ }^{1}\right.$ Div. Human Molec. Genet., Med. Inst. Bioregulation, Kyushu Univ., ${ }^{2}$ Div. Respirology, Neurol and Rheumatol., Dept. Med., Kurume Univ. Sch. Med., ${ }^{3}$ Dept. Food and Nutrition, Beppu Univ. Jounior Coll., ${ }^{4}$ Dept. Med. Chem., Hamamatsu Univ. Sch. Medicine, ${ }^{5}$ Dept. Med. Chem., Kurume Univ. Sch. Med.)

Identification of causal genetic variants associated with a clinically new type of hereditary motor and sensory neuropathy by Linkage analysis and Exome sequencing

We previously reported a clinically new type of autosomal dominant disorders of motor and sensory neuropathy with proximal dominancy in the lower extremities, urinary disturbance, and paroxysmal dry cough (Miura et al. J Neurol Sci 2008). To identify the causative mutation for the disease, we studied this pedigree consisting of 19 family members including 9 patients in 5 generations. On a multipoint linkage analysis, we observed a single peak of LOD scores on 1p13.3-q23 (LOD = 2.24). The exome sequencing revealed 1,335 patient-specific single nucleotide variants (SNVs). Through the validation, we confirmed cosegregation of 2 candidate SNVs in the pedigree, and through the functional predictions of the cosegregated SNVs, we conduced the SNV located in IQGAP3 is highly likely to be the causative variant for the disease. This SNV is located within 5' splice site consensus sequence in intron 27 in IQGAP3, potentially affecting the IQGAP3 splicing. The SNV is also located within a non-coding RNA (ENST00000476565). The effect of the SNV is under consideration through detecting splice variants or examining the function of the non-coding RNA.
3C MORIKAWA, Takuya ${ }^{1}$, MIURA, Shiroh ${ }^{2}$, FUJIOKA, -09 Ryuta $^{3}$, KOSAKA, Kengo ${ }^{1}$, YAMADA, Kohei ${ }^{1}$, HATTORI, Gohsuke ${ }^{4}$, MOTOMURA, Manabu ${ }^{5}$, TANIWAKI, Takayuki ${ }^{2}$, SHIBATA, Hiroki ${ }^{1}\left({ }^{1}\right.$ Div. Genomics, Med. Inst. Bioregulation, Kyushu Univ., ${ }^{2}$ Div. Respirol., Neurol, and Rheumatol., Dept. Med., Kurume Univ. Sch. Med., ${ }^{3}$ Dept. Food and Nutr., Beppu Univ. Junior Coll., ${ }^{4}$ Dept. Neurosurg., Kurume Univ. Sch. Med., ${ }^{5}$ Dept. Internal Med., Nagasaki Yurino Hosp.)

Homozygous 4-bp deletion in the $D D H D 1$ gene, resulting the complete deletion of DDHD domain, as a causative variant in a SPG28 patient

Spastic paraplegia (SPG) type 28 is an autosomal recessive hereditary SPG caused by mutations in the $D D H D 1$ gene. We examined a Japanese 54-years-old male patient with autosomal recessive SPG. By the exome sequencing analysis, we identified 17,248 homozygous nucleotide variants in the patient. Through the examination of 47 candidate genes known to be responsible for autosomal recessive SPG, we identified a novel homozygous 4bp deletion c914_917delGTAA,Ser305Ilefs*2 in exon2 of the DDHD1 encoding phosphatidic acid-preferring phospholipase A1 (PA-PLA1). The mutation is expected to cause a frameshift generating premature stop codon at 3-bp downstream from the deletion. In consequence, the DDHD domain that is known to be critical for PLA1 activity is completely depleted in the mutated DDHD1 protein, resulting the practically a null mutation of the $D D H D 1$. By Sanger sequencing, we confirmed that both parents are heterozygous for the mutation. This variation was not detected in 474 Japanese control subjects. We conclude that the novel mutation in DDHD1 is the causative variant for the SPG28 patient that is the first record of the disease in Asian population.

3C IKEUCHI, Tatsuro ${ }^{1}\left({ }^{1}\right.$ Formerly, Med. Res. Inst., -10 Tokyo Med. and Dental Univ.)

High school textbooks of "Seibutsu-kiso (Basis of Biology)" under the MEXT New Course of Study: with special reference to the new edition after the 2016 examination procedure by MEXT

There have been two main problems to be improved in the high school "biology" textbooks under the new education guidelines offered in 2012 by the Ministry of Education, Culture, Sports, and technology (MEXT). One is that the topic pertaining to the "Mendel's laws of inheritance" was moved from the high schools to the junior high school curriculums, and is now not included in high school biology textbooks. The second is the insufficient description for explaining the mechanism of human heredity. Recently, the new editions of high school textbooks of "Seibutsukiso (Basis of Biology)" were presented after the 2016 examination procedure by MEXT. Contrary to our expectation there were no sufficient improvements about the above mentioned problems. Outlines of the new edition were introduced. It should be again emphasized that the Mendel's laws of inheritance is fundamental and indispensable for understanding the modern genetics, and that a basic knowledge of human heredity is essential for facing it in the "personal genome era" that we are now entering. 

-01 ('ife Sci., Grad. Sch. Sci. and Eng., Tokyo Metropolitan Univ.)

Changes in gut microbiota at fungal infection in the Drosophila melanogaster species group

Microbial resistance of Drosophila species varies among different species as Drosophila adapted to various microbial environments species by species. For example, $D$. virilis inhabits fungi rich environments, whereas $D$. melanogaster inhabits yeast rich environments. Accordingly, $D$. virilis survives longer in a culture with Penicillium fungi than $D$. melanogaster does. However, overproduction of antimicrobial peptides that play an important role in microbial resistance in gut alters gut microbiota to shorten the host fly longevity, suggesting that stability of normal gut microbiota is responsible for the microbial resistance. In this study, to clarify whether the gut microbiota is responsible for the microbial resistance, we examined gut microbiota for four Drosophila species, sequencing $16 \mathrm{~S}$ ribosomal DNA and a survival rate with Penicillium sp. As a result, we found that gut microbiota in all four species was distorted by the ingestion of Penicillium fungi to decline the survival rate during lava to adult. Therefore, the maintenance of gut microbiota by antimicrobial peptides is expected to be a critical factor of the survival of Drosophila species.

3D SETO, Yosuke ${ }^{1}$, TAMURA, Koichiro ${ }^{1}$ ( ${ }^{1}$ Dept. Biol. -02 Sci.s, Sch. Sci. Eng., Tokyo Metropolitan Univ.)

Comparative gene expression study of antifungal immune response among Drosophila species

Drosophila flies are exposed to infection of various microorganisms from their digestive organs, as they feed and breed on fermented fruits and sap, where a variety of fungi propagate. They defend themselves from invading microorganisms with innate immune system. In this study, we use three Drosophila species which show different food preference. Drosophila melanogaster, $D$. virilis and $D$. brachynephros utilize fermented fruits, sap and mushrooms, respectively. To clarify the antifungal immune system responsible for the difference of food preference, we compared gene expression patterns in gut and in fat body between the three species in response to the infection of Penicillium fungus. As the results, antimicrobial peptides and lysozymes synergistically act against the infected fungi in $D$. virilis and $D$. brachynephros unlike the antifungal immune response in the fat body of $D$. melanogaster. Additionally, expressions of many serine protease genes were up-regulated in both the infected $D$. virilis and $D$. brachynephros. These results indicate that the immune system has been substantially differentiated between Drosophila species having different ecological characters.
3D TATSUMOTO, Shoji ${ }^{1}$, TOYODA, Atsushi ${ }^{4}$, IMAI, -03 Hiroo $^{5}$, HIRAI, Hirohisa ${ }^{5}$, YAMAMORI, Tetsuo ${ }^{6}$, ISA, Tadashi $^{7}$, FUJIYAMA, Asao ${ }^{4}$, GO, Yasuhiro ${ }^{1,2,3}$ $\left({ }^{1}\right.$ Dept. Brain Sci.s, Cent. Novel Sci. Initiatives, Natl. Inst. Natural Sci., ${ }^{2}$ Dept. System NeuroSci., Natl. Inst. Physiological Sci., ${ }^{3}$ Dept. Physiol. Sci., Grad. Sch. Life Sci., SOKENDAI (Grad. Univ. Adv. Stud.), ${ }^{4}$ Comp. Genomics Lab., Natl. Inst. Genet., ${ }^{5}$ Mol. Biol. Section, Primate Res. Inst., Kyoto Univ., ${ }^{6}$ Mol. Anal. for Higher Brain Funct., Brain Sci. Inst., RIKEN, ${ }^{7}$ Dept. NeuroSci., Grad. Sch. Med. and Faclt. Med., Kyoto Univ.)

Population exome analysis in macaques reveals evolutionary trajectories of Japanese monkeys

To reveal evolutionary histories of Japanese monkeys, we performed whole exome sequences (WES) in 77 Japanese monkeys as well as 13 Chinese and 8 Indian rhesus monkeys as outgroups. We obtained the high-quality reads that cover $95.5 \%$ of the target region $(53.7 \mathrm{Mb})$ with at least one read and identified about 345K SNPs. Principal component analysis (PCA) clearly shows three distinct clusters of Japanese and two populations (Chinese and Indian) of rhesus monkeys. Moreover, a clustering analysis shows that Chinese and Indian rhesus monkeys are making sister groups, which is not consistent with previous works that insisted more closely relationships between Japanese and Chinese rhesus monkeys. In addition, we found that Japanese monkey evolutionary and functionally specific genes are enriched in the genes involved in the fatty acid synthesis, skeletal development, brain function, immune response, sperm mortality, and chemosensory perception.

3D MATSUSHITA, Yuka ${ }^{1}$, TAKEZAKI, Naoko ${ }^{2}$, MELIN, -04 Amanda Dawn ${ }^{3}$, KAWAMURA, Shoji ${ }^{1}{ }^{1}$ Dept. Integr. BioSci.s, Grad. Sch. Front. Sci.s, The Univ. Tokyo, ${ }^{2}$ Life Sci. Res. Cent., Kagawa Univ., ${ }^{3}$ Dept. Anthropol. \& Archaeol., Univ. of Calgary)

L-M opsin nucleotide divergence depicts contrasting selective modes between trichromatic New World howler monkey and Old World primates

In New World monkeys, howler monkeys (Alouatta) are the only genus having $\mathrm{L}$ and $\mathrm{M}$ opsin genes juxtaposed on the $\mathrm{X}$ chromosome. It is reported that they also have a high frequency of L/M hybrid opsin genes, which diverge from normal L and M opsins in absorption spectra. A previous study showed that in Old World primates, excepting humans, the spectral difference between $\mathrm{L}$ and $\mathrm{M}$ opsins is maintained by purifying selection against homogenization due to gene conversion in central exons. However, it is not known whether such homogenization and purifying selection are also operating in Alouatta. Here, we use target capture and next-generation sequencing (NGS) to examine nucleotide divergence between Alouatta L and M opsin genes including all introns. Contrasting to Old World primates, the L and $\mathrm{M}$ opsin genes showed high nucleotide divergence throughout the gene region including introns. Thus, homogenization due to gene conversion is not obvious. Nevertheless, Alouatta species have L/M hybrid opsin genes at high frequency. This suggests a distinct mode of selection in Alouatta from Old World primates allowing color vision variation as in other New World monkeys. 
3D KAWAMURA, Shoji ${ }^{1}$, KASAGI, Satosi ${ }^{1,2}$, KASAI, -05 Daisuke ${ }^{1}$, TEZUKA, Ayumi ${ }^{3}$, SHOJI, Ayako, TAKAHASHI, Akiyoshi ${ }^{2}$, IMAI, Hiroo ${ }^{4}$, KAWATA, Masakado $^{3}\left({ }^{1}\right.$ Dept. Integr. BioSci.s, Grad. Sch. Front. Sci.s, The Univ. Tokyo, ${ }^{2}$ Sch. Marine Biosci.s, Kitasato Univ., ${ }^{3}$ Grad. Sch. life Sci., Tohoku Univ., ${ }^{4}$ Primate Res. Inst., Kyoto Univ.)

Spectral sensitivity of guppy visual pigments reconstituted in vitro to resolve association of opsins with cone cell types

The guppy (Poecilia reticulate) shows remarkable variation of photoreceptor cells in the retina. Microspectrophotometry (MSP) has revealed varying green, green-yellow and yellow cone cells among guppies in Trinidad and Venezuela (Cumana). In the guppy genome, there are four long-wave opsin loci. Two LWS-1 alleles have potentially differing spectral sensitivity. However, the absorption spectra of these photopigments have not been measured directly and the association of cell types with these opsins remains speculative. In the present study, we reconstituted these opsin photopigments in vitro. The wavelengths of maximal absorbance (max) of LWS-3 was much shorter than the value expected from the five-sites" rule. The two LWS-1 alleles could explain difference of the reported MSP max values for the yellow cone class between Trinidad and Cumana guppies. Absence of the short-wave-shifted LWS-3 and the green-yellow cone in the green swordtail supports the hypothesis that this cell class of the guppy co-expresses the LWS-1 and LWS-3. These results reveal the basis of variability in the guppy color vision."

3D IIZUKA, Tomoyo ${ }^{1}$, NOZAWA, Masafumi ${ }^{2}$, IKEO, -06 Kazuho ${ }^{1,3}$ ( ${ }^{1}$ Dept. Genet., SOKENDAI, ${ }^{2}$ Tokyo Metropolitan Univ., ${ }^{3}$ Natl. Inst. Genet.)

Prediction of the responsible gene that determines the morphological characters of septal pore cap, the specific structure in Agaricomycetes

Identifying the gene that corresponds to the morphological diversity is important to know the genetic basis of evolution of morphology. In this study, we focus on the morphological characters of septal pore cap (SPC); component of cell-to-cell junction of Agaricomycotina(Fungi).

The phylogenetic tree shows that "vesiculate" SPC (vSPC) is most ancestral type of SPC and the perforate" SPC (pSPC) had emerged multiple times from "imperforate" SPC (iSPC).

\section{D OOTA, Satoshi ${ }^{1}\left({ }^{1}\right.$ RIKEN BioRes. Cent.) $-07$}

ThreeTree: a metric-based method to reconstruct phylogenetic trees

Reconstruction of phylogenetic trees is equivalent to map the evolutionary distance space to the two-dimensional tree graph space. It is well known that the pair-wise evolutionary distance space often violates metricity. This is one of the main causes for misinference of phylogenetic trees. Meanwhile, virtually all existing phylogenetic reconstruction methods follow the minimum evolution (ME) principle. The ThreeTree method is a new kind of approach to reconstruct phylogenetic trees based on the optimal metricity of the evolutionary distance space rather than the ME principle. This method can neatly find evolutionarily plausible topologies even from phylogenetically ill-conditioned data.

3D IWAMOTO, Eisuke ${ }^{1}$, TAMURA, Koichiro ${ }^{1,2}\left({ }^{1}\right.$ Dept. -08 Biol. Sci.s, Tokyo Metro. Univ., ${ }^{2}$ Res. Cent. Genomics and Bioinformatics)

Sorting and filtering sequence data makes phylogenomic tree estimation

better

In molecular phylogenetic analyses, a phylogenetic tree is reconstructed from molecular sequence data based on a mathematical model of molecular evolution. Today, most of the treereconstruction methods assume that the pattern of evolution is constant through all the concatenated genes. When this assumption is violated, the accuracy of phylogenetic tree reconstruction may deteriorate. In this study, we developed a method to improve the accuracy of phylogenetic tree reconstruction by removing the genes that cause errors in phylogenetic tree reconstruction.

We first sorted genes by means of the similarities between distance matrices of the genes, and then decided the amount of data removed according to the difference between the observed distance matrix and the patristic distance matrix obtained from the inferred phylogenetic tree. We tested this method by using the honey bee dataset (Woodard et al. 2011), which was reported to cause erroneous phylogenetic tree-reconstruction by base composition bias (Romiguier et al. 2016). We found that the method improved the accuracy of phylogenetic tree reconstruction. 
$3 D$ $-09$

ENDO, Toshinori ${ }^{1}$ ( ${ }^{1}$ Grad. Sch. Inform. Sci. Tech.,

Extraction and comparative analysis of genes over levels of taxonomic classification in common

To address commonalities and lineage-specificities of gene sets across eukaryotes, comprehensive classification and analysis was conducted on the genomes for 10 phyla, 24 classes, 99 orders, 189 genus and 393 species publicly available at NCBI repository. The total number of sequence used accounts for 9,114,517 entries. For primary gene identification across species, provided annotation data are utilized by extracting stem functional information. All the taxa information were manually curated for classification purpose, based on Taxonomy database and other resources for the classification in question. This is because some NCBI is not authority of taxonomy and some taxonomic information did not seem reliable. Based on above efforts, genes are classified on the commonalities across each level of taxa, and then by commonality spectrum. In the presentation, I report the resulted pivotal genes shared across levels of taxa to discuss their roles in evolutionary paths.
3D WATABE, Dai ${ }^{1}$, YUASA, Hiroshi ${ }^{2}$, ENDO, Toshinori ${ }^{1}$ -11 ( ${ }^{1}$ Grad. Sch. Inform. Sci. Tech., Hokkaido Univ., ${ }^{2}$ The Res. Inst. of Evolutionary Biol.)

Estimation of the bottle gourd propagation route by molecular analysis

Bottle gourds distribute worldwide. It is considered one of the oldest cultivated plants, probably native to Africa where their wild species solely exists. In this study, we analyzed DNA diversity of regional specimens of bottle gourds collected worldwide to clarify the propagation route. For this purpose, we determined sequences of three chloroplast and four nuclear DNA regions from 60 samples. The results revealed that two INDELs and three SNPs in chloroplast consistently separated African and Asian types except Ethiopian being classified as Asian. For two INDELs, two previous studies contradicted. Our results supported Erickson et al. (2005) who suggested American bottle gourds arrived via Asia. The nuclear variations also separated African and Asian consistent with the chloroplast results. Interestingly, a Guatemalan sample belonged to the Asian variation and the rest of American samples were hybrids. Our results supported that the ancient propagation was via Asia based on DNA typing.
3D TAKEZAKI, Naoko ${ }^{1}$, NISHIHARA, Hidenori ${ }^{2}\left({ }^{1}\right.$ Life -10 Sci. Res. Cent., Kagawa Univ., ${ }^{2} 2$ Grad. Sch. BioSci. and BioTech., Tokyo Inst. Tech.)

Phylogenetic analysis of coelacanth, lungfish, and tetrapods with slowly evolving ray-finned fish as the outgroup

In our previous analysis of the relationships of coelacanths, lungfishes and tetrapods, with cartilaginous fish as the outgroup, the sister relationship between lungfish and tetrapods was constructed with high statistical support. However, with rayfinned fish as the outgroup, the sister relationship of coelacanth and tetrapods was mostly constructed, though the statistical support tended to be low. Our study indicated that the tree topology was likely to be distorted because of the high divergence of the ray-finned fish sequences. This study shows that with rayfinned fish with slow evolutionary rate, gar and bowfin, as the outgroup, the sister relationship of lungfishes and tetrapods was reconstructed with high statistical support.

\section{D TAKEMURA, Toshihiko ${ }^{1}\left({ }^{1}\right.$ Takemura Lab.) $-12$}

On the influence of the water molecular undulation for the creation of the macromolecules in the lives body

From the great many hydrogen bonds which are led in the many parts of the lives body created the adsorbed heavy water [H3O] what lead the LASER gravity through a series of the energy accumulating reactions, which are the antagonistic rays of the increased intermolecular gravity and the scatter rays as the incident rays of the resonance rays of the hydrogen which by the reflected repulsion of the hydrogen in the reverse distribution space, what are strong coherent force and diffuse so reflect from inner to the body cuticle repeatedly so diffused all over the body and the body become the light reverse distribution space, what create the great many virtual images on the line of the each radiation and filled up the proper energy levels so recreate the great many hydrogen bond colonies scattering in the lives body. These great many hydrogen bonds colonies on the virtual images filled up the many matters and become the many macromolecules in the lives body, what reaction are led through the water adsorbed the matter (M-H2O)by the substitution.

For reference the T. Takemura scattering. 
3E YOSHIDA, Takanori ${ }^{1}, \mathrm{KAWABE}$, Akira $^{1}\left({ }^{1}\right.$ Faclt. Life -01 Sci.s, Kyoto Sangyo Univ.)

De novo genome assembly of tower mustard, Turritis glabra

Genome rearrangements such as inversions and chromosome fusions are one of the major driving forces of genome evolutions. Model plant Arabidopsis thaliana and its relative species had experienced several chromosome rearrangements (Lysak et al. 2006). Although the outline of rearrangements have been investigated by comparative chromosome paintings, the details of breakpoints are still not clear for many relative species because of the lack of precise genome assemblies. The genome assembly based on the single-molecule real-time sequencing can improve draft genomes and provide many insights into the details of chromosome rearrangements. We conducted sequencing by PacBio sequencer and de novo assembly of Turritis glabra, a relative of $A$. thaliana. Compared with ancestral karyotype, $T$. glabra have reduced number of chromosomes because of chromosome rearrangements (Lysak et al. 2016). The draft genome had 594 contigs with N50 of $563 \mathrm{~Kb}$. We found major breakpoints of rearrangement at chromosome 2 and 4 by comparing with synteny block of A. thaliana. In future, the molecular evolution of flanking genes should be analyzed to reveal the effect of chromosome rearrangements.

$3 \mathrm{E}$

NAITO, Ken ${ }^{1}$, SAKAI, Hiroaki ${ }^{1}$, TAKAHASHI, Yu ${ }^{1}$, -02 ISEKI, Kohtaro ${ }^{2}$, MUTO, Chiaki ${ }^{1}$, KAGA, Akito ${ }^{1}$, TOMOOKA, Norihiko ${ }^{1}$ ( ${ }^{1}$ NATO Genetic Resource Center, ${ }^{2}$ Japan InterNatl. Res. Cent. of Agricultural Sci.)

De novo assembly of whole genome sequences of the wild species of genus Vigna by PacBio sequencer

Genus Vigna consists of legume crops such as azuki bean as well as a number of wild species. One of the most attractive features of the genus Vigna is the great adaptability of the wild species to harsh environmental conditions. By figuring out the mechanisms of how they adapt to such environments, it would be possible to generate novel crops tolerant to the stress conditions. Genome information is of great importance to promote such researches. Thus, in order to establish the genome basis of genus Vigna, we launched the Vigna genome project.

In this study, we adopted single molecule real time (SMRT) sequencing because of its read length. We sequenced the 10 wild species which has great tolerance against stresses of high salt, acidic soil, alkaline soil, drought or flooding. The genome sizes ranged from $480 \mathrm{Mb}$ to $780 \mathrm{Mb}$, the sequencing depth ranged from $20 \mathrm{x}$ to $60 \mathrm{x}$. The assembly stats achieved contig N50 of more than $3.0 \mathrm{Mb}$ in several species.
3E NAKAMURA, Chiharu ${ }^{1}$, TAKENAKA, Shotaro ${ }^{1}$ -03 ( ${ }^{1}$ Dept. Plant Life Sci., Faclt. Agr., Ryukoku Univ.)

Cytoplasmic diversity affecting submergence stress responses in wheat

Plant cells possess three different genomes, i.e, nuclear, mitochondrial and chloroplast genomes, in their respective cellular compartments. These genomes have been known to interact to maintain plant homeostasis during its whole developmental stages. In the Triticum-Aegilops complex, cytoplasmic genome diversity and nucleus-cytoplasm (NC) genome interactions affecting various phenotypes can effectively be studied using a series of NC hybrids in which particular nuclear genomes of wheat are combined with various cytoplasmic genomes of Aegilops species. We evaluated submergence stress responses in $39 \mathrm{NC}$ hybrids at germination and the early seedling stages. Bioassays revealed a large variability among them. Some Aegilops cytoplasms either enhanced or reduced the sensitivity to submergence stress of NC hybrids as compared with that of their nuclear donor. Because all NC hybrids possessed an identical nuclear genome, observed variability can be ascribed to cytoplasmic genome differences and further suggests an involvement of retrograde regulation by that different cytoplasmic genomes regulate expression of the donor nuclear genome under this important abiotic stress in wheat.

3E TAKADA, Toyoyuki ${ }^{1}, \mathrm{KONDO}, \mathrm{Shinji}^{2}$, YASAKA, -04 Taku ${ }^{3}$, ABE, Takashi ${ }^{3}$, KIYOSAWA, Hidenori ${ }^{4}$, SUZUKI, Yutaka ${ }^{5}$, ATSUSHI, Toyoda ${ }^{6}$, FUJIYAMA, $\mathrm{Asao}^{2,6}$, SHIROISHI, Toshihiko ${ }^{1}$ ( ${ }^{1}$ Mammalian Genet. Lab., Genetic Strains Res. Cent., Natl. Inst. Genet., Mishima, ${ }^{2}$ Cent. Genome Inform, Res. Org. Inform. Systems, ${ }^{3}$ Dept. Inform Eng., Niigata Univ.rsity, ${ }^{4}$ Dept. Environ. Med., Kochi Med. School, Kochi Univ., ${ }^{5}$ Dept. Comput. Biol. and Med. Sci., Grad. Sch. Front. Sci.s, The Univ. Tokyo, ${ }^{6}$ Comp. Genomics Lab., Natl. Inst. Genet.)

Comparative analysis of gene expression polymorphisms based on mouse inter-subspecific genome divergence

Mammalian complex traits are determined by genetic and environmental factors along with sex and aging of the individuals. The genetic factors include variation of gene expression levels as well as amino acid substitutions. We intend to survey the intersubspecific differences of the gene expression levels using Japanese wild mice-derived inbred strain MSM/Ms and most commonly used inbred strain C57BL/6J. MSM/Ms strain shows highly extent of phenotypic variations in many complex traits and vast amount of genome diversity for C57BL/6J. At the beginning, we have started genome-wide transcriptome analysis of liver samples, because it is inferred that wild-derived MSM/Ms strain has regionally adapted unique energy metabolic pathway between commonly used inbred strains. In this talk, we will show recent results of liver transcriptome analysis of C57BL/6J, MSM/ $\mathrm{Ms}$ and experimental cross strains generated from them. 
3E FURUYAMA, Masahiro ${ }^{2}$, SATO, Tadashi ${ }^{1}$, MYOSHO, -05 Taijun ${ }^{1}$, SAKAIZUMI, Mitsuru ${ }^{1}$ ( ${ }^{1}$ Faclt. Sci., Niigata Univ., ${ }^{2}$ Grad. Sch. Sci. and Tech., Niigata Univ.)

Gene-centromere mapping in the medaka fish using halftetrad analysis

Gene-centromere mapping in medaka using half-tetrad analysis Masahiro Furuyama, Tadashi Sato, Taijun Myosyo, Mitsuru Sakaizumi

Although gene-centromere mapping provides important resources for genetics and comparative genomic studies, it has not been reported in medaka. Medaka has 24 pairs of chromosomes consisting of 10 pairs of biarmed and 14 pairs of monoarmed chromosomes. Fluorescent in situ hybridization (FISH) analyses have shown that linkage group (LG)1, 9 and 18 are biarmed (Matsuda et al., 2002, Brunner et al., 2001 and Kondo et al., 2002) and LG11 and 13 are monoarmed (Myosho et al., 2012). In this study we performed half-tetrad analysis using F1 hybrid females between two inbred strains, Hd-rR (Oryzias latipes) and HNI (O. sakaizumii) and males of an inbred strain of $\mathrm{O}$. luzonensis. A total of 141 DNA markers were selected across 24 LGs. Genotyping of 112 triploid hybrids obtained by inhibiting the release of the second polar body of oocytes localized all 24 centromeres onto their respective LGs according to their closest markers. We propose here that 10 LGs $(1,2,4,9,12,14,18,19,21$ and 23) are biarmed and the remaining 14 LGs are monoarmed chromosomes.

3E NAGAOKA, Haruna ${ }^{1}$, MYOSHO, Taijun ${ }^{3}$, SATO, -06 Tadashi ${ }^{2}$, SAKAIZUMI, Mitsuru ${ }^{2}\left({ }^{1}\right.$ Grad. Sch. Sci. and Tech., Niigata Univ., ${ }^{2}$ Inst. Sci. Tech., Acad. Assembly, Niigata Univ.rsity, ${ }^{3}$ Sch. Food and Nutritional Sci., Univ. of Shizuoka)

Lethality Caused by Substitution of the Chromosome Between Medaka Strains

It lies at the heart of speciation to reveal hybrid incompatibility between related species. Japanese medakas, Oryzias latipes and O. sakaizumii, have parapatric distribution in Japan. Inbred strains have been established from both species, such as Hd-rR from O. latipes and HNI from O. sakaizumii. They can mate and produce fertile progeny. Consomic embryos, Hd-rR-Chr23 ${ }^{\mathrm{HNI}}$ which carry the HNI Chr23 in the Hd-rR background, develop abnormally, and die before hatch. This suggests a genetic incompatibility of the HNI Chr23 and Hd-rR background chromosome (s). A causal factor on Chr23 was mapped near a telomere. To search for responsible factors in the Hd-rR background, we crossed Hd-rR-Chr23 $3^{\mathrm{HNI} / \mathrm{Hd}-\mathrm{rR}}$, which is heterozygous for $\mathrm{Chr} 23$, to $(\mathrm{HNI} \times \mathrm{Hd}-\mathrm{rR}) \mathrm{F} 1$, and obtained 28 progeny homozygous for HNI Chr23. All the 28 progeny had heterozygous genotype for a centromeric marker on Chr20, suggesting another responsible factor on Hd-rR Chr20. Recombinant breakpoint analyses restricted the region of two responsible factors to $23.86 \mathrm{Mbp}$ end of $\mathrm{Chr} 23$ and to $0 \sim 3.1 \mathrm{Mbp}$ of $\mathrm{Chr} 20$ on the medaka physical map.
3E KINJO, Sonoko ${ }^{1}$, YAGUCHI, Shunsuke ${ }^{2}$, YAMAMOTO -07 Takashi $^{3}$, KIYOMOTO, Masato ${ }^{4}$, IKEO, Kazuho ${ }^{1}$ $\left({ }^{1}\right.$ Cent. Inform. Biol., Natl. Inst. Genet., ${ }^{2}$ Shimoda Marine Res. Cent., Univ. of Tsukuba, ${ }^{3}$ Dept. Math. and Life Sci.s, Grad. Sch. Sci., Hiroshima Univ., ${ }^{4}$ Tateyama Marine Lab., Marine and Coastal Res. Center, Ochanomizu Univ.)

A genome and transcriptome database of a sea urchin Hemicentrotus pulcherrimus created by NGS data analysis platform, MASER

We developed and utilized a next-generation sequencing (NGS) data analysis platform, MASER (Management and Analysis System for Enormous Reads) for analyzing genome and transcriptome data of sea urchin Hemicentrotus pulcherrimus which has a long history as a model organism in the field of developmental and cell biology since the mid 1900s. MASER allows us not only to analyze NGS data but also share the resultant data with public users or limited users for some of analyses. By applying this system to a genome of $H$. pulcherrimus, we obtained a total of 16,251 scaffold sequences with N50 length of $143 \mathrm{k} \mathrm{bp}$ and identified approximately 25,000 genes. The size of genome was estimated to be around $800 \mathrm{M} \mathrm{bp}$. The resultant sequences and annotation information are deposited in the web site. This study is a first model case for de novo assembly and public release of eukaryotic genome easily and effectively by using MASER (http://cell-innovation.nig.ac.jp/public/contents/ service_en.html).

3E MASUYA, Hiroshi ${ }^{1}$, TAKATSUKI, Terue ${ }^{1}$, SAITO, -08 Mikako $^{1}$, TAKAYAMA, Eiki ${ }^{1}$, OHSHIMA, Kazuya ${ }^{1}$, TANAKA, Nobuhiko ${ }^{1}\left({ }^{1}\right.$ Tech. and Dev. unit for knowledge base of mouse phenotype, RIKEN BioRes. Cent.)

J-phenoeme: a database portal for phenotype information

In the use of information in the medical science, utility of the concept of "phenotype" becomes realized. For example, diseasegene relationships will be enriched by the enhancement of mutation-phenotype relationship across species, because disease composed of multiple phenotypes. We are working on the trial of the integration and wider dissemination of phenotype data from domestic bio-resource centers, such as mouse strains from Riken BRC, consomic mouse strains from NIG, NBRP rat, NBRP medaka and data of International Mouse Phenotyping Consortim. These data are linked from the database portal, J-phenome (http://jphenome.jp). In the integrated database, strains, phenotype data and mutations are described with IDs of worldstandard and can be used in linked data from these IDs. 
3E UENO, Michimune ${ }^{1}$, MATSUMOTO, Kouji ${ }^{1}$, -09 MATSUOKA, Satoshi ${ }^{1}$, HARA, Hiroshi ${ }^{1} \quad\left({ }^{1}\right.$ Dept. Biochem. Mol. Biol., Programs in Life Sci., Grad. Sch. Sci. Eng., Saitama Univ.)

An attempt to search B.subtilis gene that rescues lethality of E.coli strain lacking PE/CL

Escherichia coli membranes consist of three kinds of major membrane phospholipids: phosphatidylethanolamine (PE), phosphatidylglycerol (PG), and cardiolipin (CL). Previous studies reported that an E.coli mutant lacking PE or lacking both $\mathrm{PG}$ and $\mathrm{CL}$ is viable. However an E.coli mutant lacking PE and CL, that is, a mutant having only $\mathrm{PG}$ as a major membrane lipid is inviable. By contrast, Bacillus subtilis strain that has only PG as a major membrane lipid is viable. Therefore we attempted to search for a $B$. subtilis gene that rescued lethality of the $E$. coli strain lacking PE and CL, because of the function of the gene may reveal essential roles of major membrane phospholipids in E.coli cells.
3E KANAZAWA, Miaki ${ }^{1}$, TANAKA, Naoyuki ${ }^{1}$, -11 MATSUSHIMA, Yumeka ${ }^{1}$, TAKAHASHI, Yasuhiro ${ }^{1}$ $\left({ }^{1}\right.$ Dept. Biochem. Mol. Biol., Grad. Sch. Sci. Eng., Saitama Univ.)

Genetic analysis of suppressor mutations that bypass the essential components of iron-sulfur cluster biosynthesis system

Biological assembly of iron-sulfur (Fe-S) cluster is mediated by complex systems consisting of multiple proteins. E.coli possesses two distinct systems called the ISC and SUF machineries encoded by iscSUA-hscBA-fdx-iscX and sufABCDSE, respectively. Deletion of both pathways is lethal due to the lack of the biosynthetic apparatus for Fe?S clusters. Here we report that modification of isoprenoid biosynthetic pathway can bypass the indispensability of the Fe?S cluster biosynthetic systems, and show that the ?isc ?suf double mutants can grow without detectable $\mathrm{Fe}-\mathrm{S}$ cluster-containing proteins. We also constructed a series of mutants in which each isc gene was disrupted in the deletion background of sufABCDSE. Phenotypic analysis of the mutants revealed that $\mathrm{Fdx}$ and IscA are not indispensable under anaerobic conditions. Furthermore, we found that suppressor mutations in IscU, an Fe-S scaffold protein, could bypass the essential role of the chaperone system HscA and HscB that bind specifically to IscU. The suppressor mutations are distributed throughout the IscU molecule, suggesting that they induce conformational change of IscU and mimic the role of $\mathrm{HscA} / \mathrm{HscB}$ chaperone system.

3E SATO, Mutsumi ${ }^{1}$, MAHANNA, Sally ${ }^{1}$, IMANAKA, -12 Takanobu ${ }^{2}$, ARAKI, Masatake ${ }^{1}$, OHMURAYA, Masaki $^{2}$, ARAKI, Kimi ${ }^{1}\left({ }^{1}\right.$ Inst. Resource Dev. and Anal., Kumamoto Univ., ${ }^{2}$ Dept. Genet., Hyogo Coll. of Med.)

The roles of cathepsin B, D, and L in pancreatic acinar cells

Cathepsin B, D, and L (CB, CD, and CL) are the major lysosomal proteases and are widely distributed in the cells of various mammalian tissues. These cathepsins participates in various physiological events such as regulation of programmed cell death, activation of enzymatic precursors, and metabolic degradation of intracellular proteins through autophagy. Autophagy is a major pathway for degradation of cytoplasmic proteins and organelles, and has been implicated in the pathogenesis of acute and chronic pancreatitis.

To investigate the role of $\mathrm{CB}, \mathrm{CD}$, and $\mathrm{CL}$ in pancreatic acinar cells, we generated and examined mice specifically deficient for each of CB, CD, and CL in pancreatic acinar cells. $\mathrm{CB}^{-/-}, \mathrm{CD}^{-/-}$, and $\mathrm{CL}^{-1}$ mice showed normal pancreatic development and autophagic activity in both physiological and pancreatitis conditions. Although $\mathrm{CB}^{-/-} \mathrm{L}^{-/-}$and $\mathrm{CD}^{-1-} \mathrm{L}^{-1-}$ mice were almost normal in physiological condition, the autophagic activity was impaired in the pancreas of $\mathrm{CB}^{-/-} \mathrm{D}^{-/-}$mice, and similar to autophagy deficient mice $\left(\operatorname{Atg} 5^{-1-}\right)$. We therefore conclude that $\mathrm{CB}$ and $\mathrm{CD}$ can implicated in autophagic activity within the pancreatic acinar cells. 